\title{
The Chandra Deep Fields: Lifting the Veil on Distant Active Galactic Nuclei and $\mathrm{X}$-Ray Emitting Galaxies
}

\author{
Y. Q. Xue $e^{a, b}$ \\ ${ }^{a}$ CAS Key Laboratory for Research in Galaxies and Cosmology, Department of Astronomy, University of Science and Technology of China, Hefei \\ 230026, China \\ ${ }^{b}$ School of Astronomy and Space Science, University of Science and Technology of China, Hefei 230026, China
}

\begin{abstract}
The Chandra Deep Fields (CDFs), being a major thrust among extragalactic X-ray surveys and complemented effectively by multiwavelength observations, have critically contributed to our dramatically improved characterization of the $0.5-8 \mathrm{keV}$ cosmic X-ray background sources, the vast majority of which are distant active galactic nuclei (AGNs) and starburst and normal galaxies. In this review, I highlight some recent key observational results, mostly from the CDFs, on the AGN demography, the interactions between AGNs and their host galaxies, the evolution of non-active galaxy X-ray emission, and the census of X-ray galaxy groups and clusters through cosmic time, after providing the necessary background information. I then conclude by summarizing some significant open questions and discussing future prospects for moving forward.
\end{abstract}

Keywords:

black holes, galaxies, active galactic nuclei, quasars, accretion

\section{Introduction}

\subsection{Effectiveness of extragalactic $X$-ray surveys}

Since the discovery of the cosmic X-ray background (CXRB; e.g., Giacconi et al. 1962), various major X-ray observatories have joined the efforts of resolving it into discrete cosmic sources as well as characterizing such sources, by carrying out different tiers of extragalactic $\mathrm{X}$-ray surveys that range from shallow all-sky surveys to ultradeep pencil-beam surveys. Together, these surveys, being highly complementary to each other, effectively occupy the practically-accessible half of the so-called $\mathrm{X}$-ray survey discovery space (i.e., X-ray flux limits achieved vs. solid angles covered; see Fig. 11.1 powerfully providing a comprehensive understanding of X-ray source populations in the universe, and essentially reveal that the large portion $(\approx 80 \%)$ of the CXRB up to $\approx 10 \mathrm{keV}$ can be accounted for by discrete X-ray point sources detected, among which the vast majority are accreting supermassive black holes (SMBHs; with

\footnotetext{
Email address: xuey@ustc.edu.cn (Y. Q. Xue)

${ }^{1}$ Also see Fig. 3 and Table 1 of Brandt \& Alexander (2015) for a recent demonstration and the information of additional X-ray surveys, respectively.
}

Preprint submitted to New Astronomy Reviews millions to billions of solar masses), i.e., active galactic nuclei (AGNs), in addition to normal and starburst galaxies (see, e.g., Brandt \& Hasinger 2005 for a review).

X-ray AGN surveys are arguably the most effective method of identifying highly reliable and fairly complete samples of AGNs, due to several reasons (see, e.g., Section 1.1 of Brandt \& Alexander 2015 for detailed reasoning and caveats): (1) Observationally, $\mathrm{X}$-ray emission is nearly a universal feature of optically, infrared (IR), radio-selected AGNs that are neither highly Compton-thick (CT, i.e., with neutral hydrogen column densities of $N_{\mathrm{H}} \gtrsim 1.5 \times 10^{24} \mathrm{~cm}^{-2}$; highly CT: $N_{\mathrm{H}} \gg 1.5 \times 10^{24} \mathrm{~cm}^{-2}$; e.g., Lanzuisi et al. 2015b) nor intrinsically X-ray weak (such sources are very rare; see, e.g., Wu et al. 2011; Luo et al. 2014a). Theoretically, X-ray emission can be produced in various accretion disk models for AGNs that are applicable for a wide range of mass accretion rates (from sub-Eddington to super-Eddington accretion), disk temperatures (cold vs. hot accretion flows), gas opacities (optically thick vs. thin), and geometric structures (thin vs. thick); these models invoke a corona or corona-like component to Compton up-scatter soft photons into hard X-rays when necessary (see, e.g., Yuan 


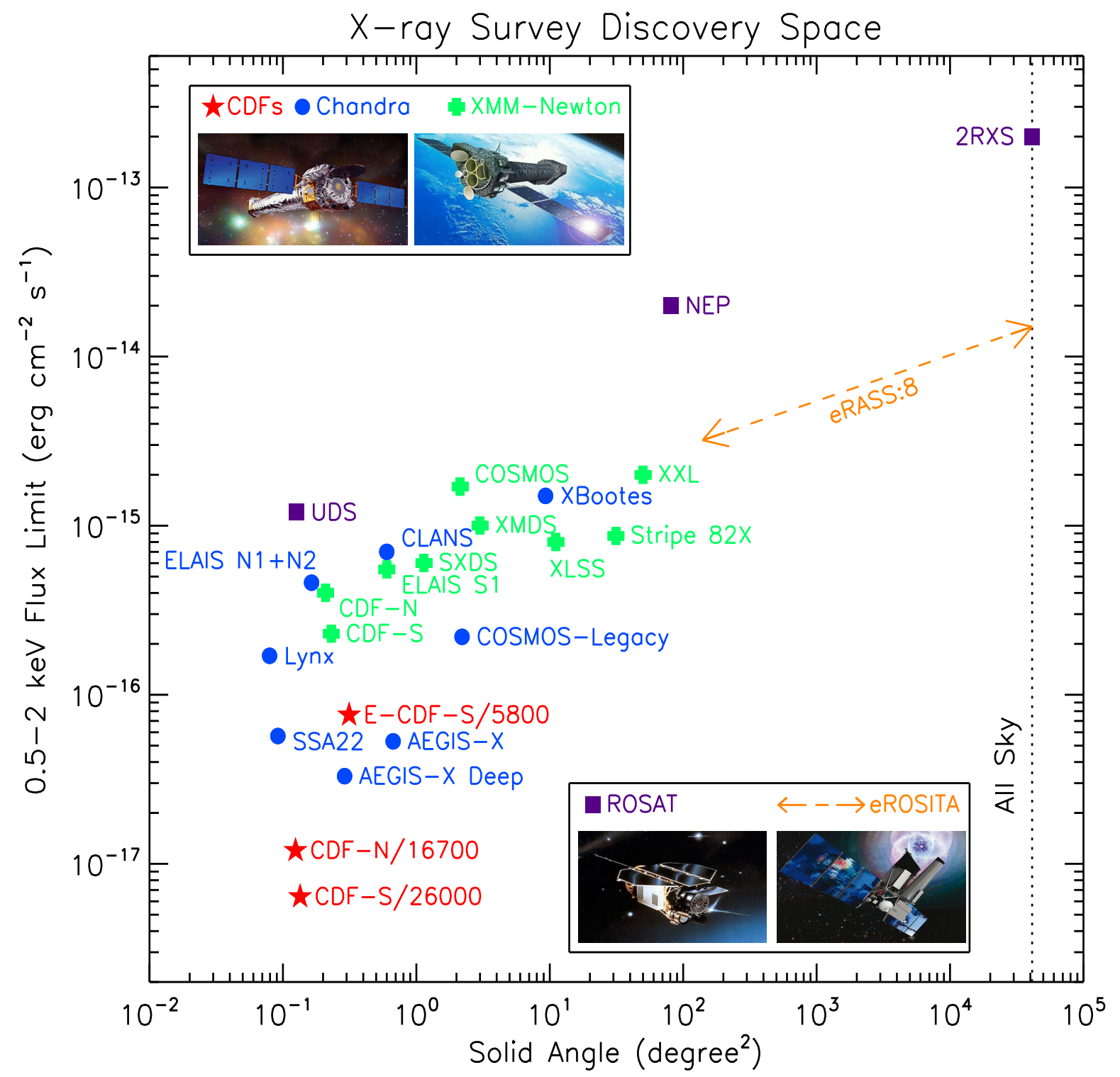

Figure 1: Flux limits achieved versus solid angles covered by some selected X-ray surveys in the $0.5-2 \mathrm{keV}$ band from Chandra, XMM-Newton, ROSAT, and eROSITA. The vertical dotted line indicates the solid angle for the whole sky. The surveys plotted are listed below, with their corresponding references shown in the parentheses: (1) for the Chandra Deep Fields (CDFs; red stars): the 7 Ms CDF-S survey (Luo et al. 2017), the 2 Ms CDF-N survey (Xue et al. 2016), and the $250 \mathrm{ks} \mathrm{E-CDF-S} \mathrm{survey} \mathrm{(Xue} \mathrm{et} \mathrm{al.} \mathrm{2016),} \mathrm{with} \mathrm{the} \mathrm{numbers} \mathrm{annotated} \mathrm{after} \mathrm{the} \mathrm{survey} \mathrm{names}$ indicating the observed X-ray source densities in their respective central 3-arcmin areas (these numbers have not been corrected for detection incompleteness or Eddington bias; see Table11 cf. Section 2.1); (2) for the other Chandra surveys (blue bullets): the Chandra Deep Survey of the Extended Groth Strip (AEGIS-X; Laird et al. 2009), the AEGIS-X Deep survey (Nandra et al. 2015), the SSA22 protocluster survey (Lehmer et al. 2009), the Lynx survey (Stern et al. 2002), the Chandra COSMOS Legacy survey (Civano et al. 2016), the ELAIS N1+N2 deep X-ray survey (Manners et al. 2003), the Chandra Lockman Area North Survey (CLANS; Trouille et al. 2008), and the X-ray survey of the NDWFS Bootes field (XBootes; Murray et al. 2005); (3) for the XMM-Newton surveys (green crosses): the CDF-S survey (Ranalli et al. 2013), the CDF-N survey (Miyaji et al. 2003), the ELAIS-S1 field survey (Puccetti et al. 2006), the Subaru/XMM-Newton Deep Survey (SXDS; Ueda et al. 2008), the XMM-Large Scale Structure survey (XLSS; Chiappetti et al. 2013), the Stripe 82X survey (LaMassa et al. 2016), the XMM Medium Deep Survey (XMDS; Chiappetti et al. 2005), the COSMOS survey (Cappelluti et al. 2009), and the XXL survey (Pierre et al. 2017); (4) for the ROSAT surveys (purple squares): the ROSAT Ultra Deep Survey (UDS; Lehmann et al. 2001); the ROSAT North Ecliptic Pole survey (NEP; Henry et al. 2006), and the Second ROSAT all-sky survey source catalog (2RXS; Boller et al. 2016); and (5) the proposed final (4 years) eROSITA All-Sky Survey (eRASS:8; the orange dashed line with two arrow heads; Merloni et al. 2012). 
\& Narayan 2014 for a review). (2) X-rays can penetrate through non-highly CT columns that are common among the majority AGN populations, and become even more penetrating at high redshifts due to positive $K$-correction, thereby reducing significantly absorption biases, probing immediate vicinities of SMBHs, and allowing for reliable $N_{\mathrm{H}}$ measurements to uncover intrinsic (i.e., absorption-corrected) AGN luminosities. (3) $\mathrm{X}$-ray emission is subject to low dilution by host-galaxy stellar emission. An X-ray point source sitting right at the center of a galaxy is very likely to be an AGN; this serves as an effective way to identify distant AGNs when it is typically unfeasible to resolve spatially AGN light from host starlight. (4) An AGN X-ray spectrum is produced through numerous line and continuum emission processes subject to obscuration, and can therefore be utilized to infer physical conditions close to the $\mathrm{SMBH}$, provided that the spectrum is of sufficient signal-to-noise ratio and energy resolution.

\subsection{The Chandra Deep Fields}

The Chandra Deep Fields (CDFs; see Fig. 2 and Table 11 consist of the Chandra Deep Field-South (CDF-S), the Chandra Deep Field-North (CDF-N), and the Extended-Chandra Deep Field-South (E-CDF-S). The CDF-S survey was originally led by R. Giacconi during 1999-2000 (1 Ms CDF-S; Giacconi et al. 2002), extended to $2 \mathrm{Ms}$ through the Director's Discretionary Time (DDT) by the CXC director H. Tananbaum in 2007 (2 Ms CDF-S; Luo et al. 2008), awarded an additional 2 Ms DDT by H. Tananbaum in 2010 (4 Ms CDF-S; Xue et al. 2011), and eventually pushed to $7 \mathrm{Ms}$ by W. N. Brandt during 2014-2016 (7 Ms CDF-S; Luo et al. 2017) ${ }^{2}$ The CDF-S patch of sky, lying in the Fornax constellation, was chosen because of very low foreground Galactic $N_{\mathrm{H}}\left(8.8 \times 10^{19} \mathrm{~cm}^{-2}\right.$; e.g., Stark et al. $1992)$, no bright $\left(m_{\mathrm{V}} \leq 14\right)$ Galactic stars, and optimal visibility from large ground-based telescopes in Chile. The CDF-N project was initiated by G. Garmire (the first $\approx 0.5 \mathrm{Ms}$ ) and W. N. Brandt (the second $\approx 0.5 \mathrm{Ms}$ ) during 1999-2001 (1 Ms CDF-N; Brandt et al. 2001), and subsequently enlarged by W. N. Brandt during 2001-2002 (2 Ms CDF-N; Alexander et al. 2003; Xue et al. 2016) ${ }^{3}$ The CDF-N lies in the Ursa Major con-

\footnotetext{
${ }^{2}$ In addition to the $7 \mathrm{Ms}$ Chandra $\approx 0.3-8 \mathrm{keV}$ CDF-S coverage, there are $\approx 3 \mathrm{Ms}$ of CDF-S coverage with XMM-Newton at $\approx 0.2-12 \mathrm{keV}$ (Comastri et al. 2011; Ranalli et al. 2013; see Fig. 1 ; and $200 \mathrm{ks}$ of E-CDF-S coverage with NUSTAR at 3-24 keV (Mullaney et al. 2015a).

${ }^{3}$ In addition to the $2 \mathrm{Ms}$ Chandra CDF-N coverage, there are 180 ks of CDF-N coverage with XMM-Newton (Miyaji et al. 2003;
}

stellation, and was chosen for largely similar considerations as the CDF-S (e.g., $N_{\mathrm{H}}=1.6 \times 10^{20} \mathrm{~cm}^{-2}$; Stark et al. 1992), in addition to the desire to cover the HDF-N that was unique back then. Furthermore, as a parallel field to the CDF-S, the CDF-N doubles the number of such deep surveys, thus controlling for influence of cosmic variance and allowing for direct comparative studies between fields. The E-CDF-S survey was carried out (PI: W. N. Brandt) in 2004 (250 ks E-CDF-S; Lehmer etal. 2005; Xue etal. 2016), which significantly expands the sky coverage of the CDF-S proper with four distinct, contiguous, and flanking pointings (thus totaling $\approx 1 \mathrm{Ms}$ exposure).

All the CDF observations were performed with the Advanced CCD Imaging Spectrometer (ACIS; Garmire etal. 2003) onboard the Chandra X-ray observatory (Weisskopf et al. 2000), whose sharp point-spread function (PSF) and low background make higher sensitivities achievable with longer exposures in an efficient manner. Indeed, the $7 \mathrm{Ms}$ CDF-S and $2 \mathrm{Ms}$ CDF-N images are the two deepest $\mathrm{X}$-ray images ever taken, thus being able to explore parameter space that has never been probed by any other X-ray surveys and fulfill one of Chandra's central design goals - revealing and characterizing the sources that constitute the CXRB. Amazingly, the faintest sources detected in the $7 \mathrm{Ms}$ CDF-S have only $\approx 1$ count per 10 days; and the 7 Ms CDF-S will serve as a multi-decade Chandra legacy for advancing deep-survey science projects, owing to its unique combination of great depth and high angular resolution. The motivation of going ultradeep is at least two-fold: on one hand, ultradeep exposures accumulate sufficient photons for known (faint) sources that allow for better characterization of the AGNs producing most of cosmic accretion power via X-ray spectral and variability analyses; on the other hand, ultra-sensitivities reveal a substantial amount of new sources (typically fainter and more obscured), thereby facilitating better understanding of obscured growing SMBHs through the $z \approx 1-4$ era of massive galaxy assembly, majority AGN populations in the first galaxies, and X-ray binary (XRB) populations in cosmologically distant starburst and normal galaxies.

\subsection{Importance of multiwavelength observations}

The ultradeep multiwavelength coverage, well matched with the CDF X-ray data, is critically important for many aspects, including source identification, source classification, measurements of host-galaxy

see Fig. 11 and $200 \mathrm{ks}$ of CDF-N coverage with NuSTAR (completed in February 2016). 


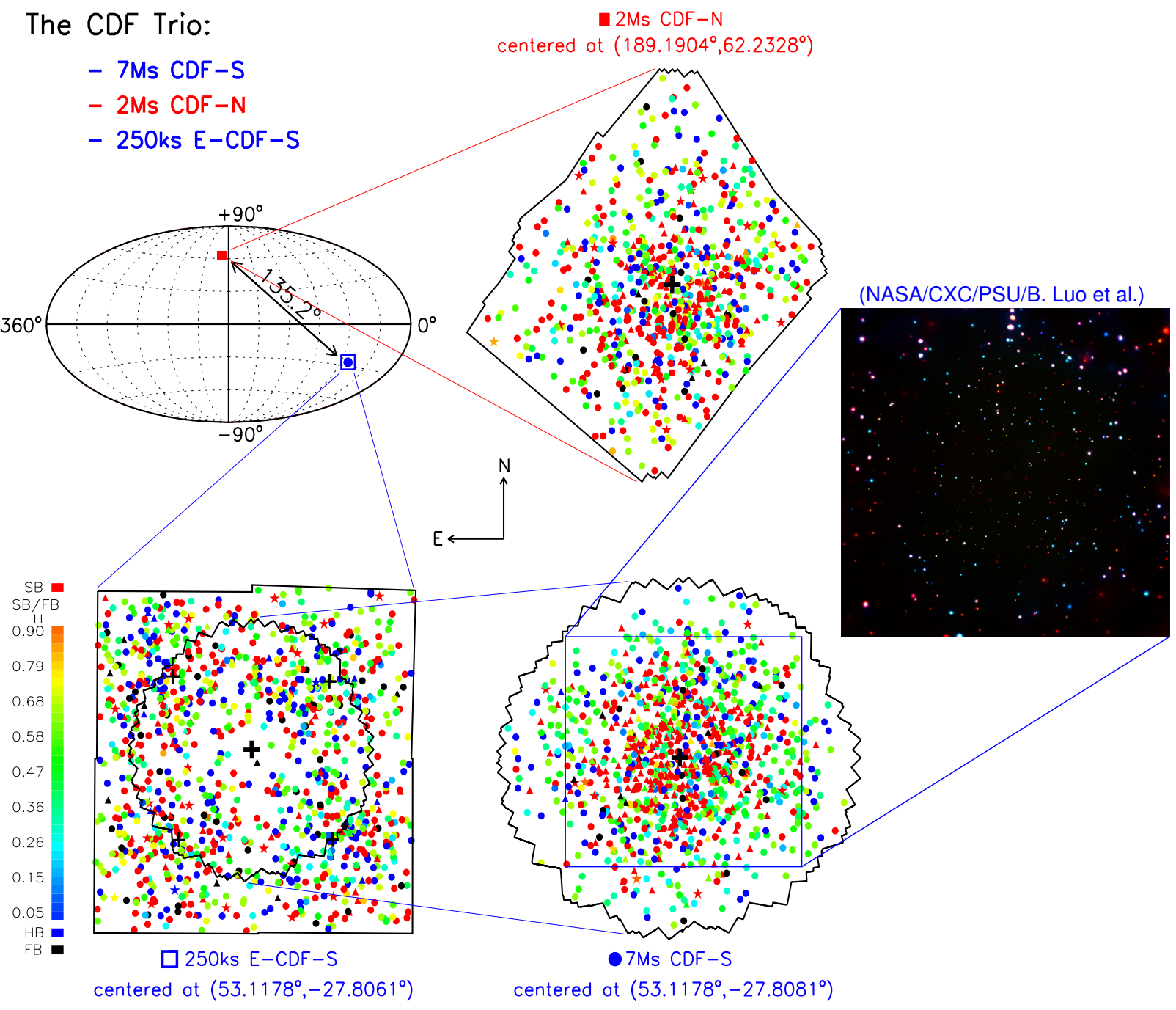

Figure 2: The CDF trio - the 7 Ms CDF-S, 2 Ms CDF-N, and 250 ks E-CDF-S (see Table 1 for more information). (Top left) The locations of the CDFs in the sky, with the separation between the CDF-N and CDF-S/E-CDF-S annotated. (Others) Spatial distributions of all point sources detected in the 2 Ms CDF-N (Xue et al. 2016), 250 ks E-CDF-S (Xue et al. 2016), and 7 Ms CDF-S (Luo et al. 2017), respectively, as well as the false-color image of the central 7 Ms CDF-S (16 arcmin across; courtesy of B. Luo). The filled circles, triangles, and five-pointed stars represent AGNs, starburst and normal galaxies, and Galactic stars, respectively. Sources detected neither in the $0.5-2 \mathrm{keV}$ band (soft band; SB) nor in the $2-7 \mathrm{keV}$ band (hard band; HB) are coded in black (i.e., detected only in the $0.5-7 \mathrm{keV}$ band; full band; FB); sources detected in the SB but not in the HB are coded in red; sources detected in the HB but not in the SB are coded in blue; and sources detected both in the SB and HB are coded in between red and blue based on ratios between the SB and SB+HB=FB net counts (see the color bar at the bottom-left corner). Bold crosses roughly indicate exposure-weighted average aim points.

physical properties (e.g., redshift, stellar mass $-M_{\star}$, star formation rate - SFR, absolute rest-frame magnitudes, morphology), and providing useful comparison samples of non-active galaxies. The CDFs are among the most intensively observed sky patches with enormous observational investments at many wavelengths from both ground and space. For instance, the CDF-S area has been covered by $\approx 50$ different photometric bands that span from extreme ultraviolet (UV) to farIR (FIR) at great depths, in conjunction with numerous deep spectroscopic and imaging observations, thereby resulting in a very-high redshift success rate $(97.8 \%$ 
Table 1: Properties of the $\mathrm{CDFs}^{a}$

\begin{tabular}{|c|c|c|c|}
\hline & CDF-S & CDF-N & E-CDF-S \\
\hline Galactic $N_{\mathrm{H}}\left(\mathrm{cm}^{-2}\right)$ & $8.8 \times 10^{19}$ & $1.6 \times 10^{20}$ & $8.8 \times 10^{19}$ \\
\hline Observational timespan & $1999 / 10-2016 / 03$ (16.4 yrs) & $1999 / 11-2002 / 02$ (2.3 yrs) & $2004 / 02-2004 / 11(0.8 \mathrm{yrs})$ \\
\hline Total number of observations & 102 & 20 & 9 \\
\hline Effective exposure (ks) & 6727 & 1896 & $235 / 209 / 240 / 241^{b}$ \\
\hline Solid angle covered $\left(\operatorname{arcmin}^{2}\right)$ & 484.2 & 447.5 & 1128.6 \\
\hline Source detection criteria & $\begin{array}{l}\text { WAVDETECT at } 10^{-5} \\
\text { and } P<0.007^{c}\end{array}$ & $\begin{array}{l}\text { WAVDETECT at } 10^{-5} \\
\text { and } P<0.004\end{array}$ & $\begin{array}{l}\text { WAVDETECT at } 10^{-5} \\
\text { and } P<0.002\end{array}$ \\
\hline Number of sources detected ${ }^{d}$ & 1008 & 683 & 1003 \\
\hline FB $(0.5-7 \mathrm{keV})$ detected counts & $(11.2,98.9,56916.2)^{e}$ & $(8.1,66.2,19748.4)$ & $(3.3,27.1,4010.6)$ \\
\hline SB $(0.5-2 \mathrm{keV})$ detected counts & $(6.1,47.4,38817.0)$ & $(5.4,35.0,14227.3)$ & $(2.2,18.9,2802.6)$ \\
\hline $\mathrm{HB}(2-7 \mathrm{keV})$ detected counts & $(9.2,94.6,18137.8)$ & $(7.7,57.5,5540.6)$ & $(3.4,20.4,1210.8)$ \\
\hline $1 \sigma$ X-ray positional uncertainty (") & $(0.11,0.47,1.28)$ & $(0.10,0.47,2.02)$ & $(0.10,0.63,1.30)$ \\
\hline Logarithm of FB flux ( $\left.\mathrm{erg} \mathrm{cm}^{-2} \mathrm{~s}^{-1}\right)$ & $(-16.76,-15.50,-12.96)$ & $(-16.35,-15.09,-12.70)$ & $(-15.73,-14.79,-12.88)$ \\
\hline Logarithm of SB flux (erg cm $\left.\mathrm{cm}^{-2} \mathrm{~s}^{-1}\right)$ & $(-17.11,-16.19,-13.29)$ & $(-16.83,-15.79,-13.07)$ & $(-16.13,-15.27,-13.26)$ \\
\hline Logarithm of HB flux ( $\left.\mathrm{erg} \mathrm{cm}^{-2} \mathrm{~s}^{-1}\right)$ & $(-16.46,-15.25,-13.13)$ & $(-16.15,-14.95,-12.95)$ & $(-15.73,-14.70,-13.02)$ \\
\hline Faintest sources detected & 1 count per $\approx 10$ days & 1 count per $\approx 4$ days & 1 count per $\approx 1$ day \\
\hline Logarithm of $L_{0.5-7 \mathrm{keV}}\left(\mathrm{erg} \mathrm{s}^{-1}\right)^{f}$ & $(39.01,42.48,45.05)$ & $(39.28,42.94,45.07)$ & $(39.89,43.34,45.50)$ \\
\hline$\%$ of multiwavelength identifications & $98.4 \%$ & $98.1 \%$ & $95.5 \%$ \\
\hline$\%$ of $z_{\text {spec }}\left(z_{\text {adopted }}\right)^{g}$ & $67.2 \%(97.8 \%)$ & $51.4 \%(93.4 \%)$ & $47.5 \%(80.8 \%)$ \\
\hline$z_{\text {adopted }} h$ & $(0.000,1.156,5.776)$ & $(0.000,1.130,5.365)$ & $(0.000,1.193,7.203)$ \\
\hline$\%$ of AGNs/galaxies/stars & $70.5 \% / 28.3 \% / 1.2 \%$ & $86.5 \% / 11.0 \% / 2.5 \%$ & $90.6 \% / 6.7 \% / 2.7 \%$ \\
\hline AGN/galaxy/star density $\left(\mathrm{deg}^{-2}\right)^{i}$ & $13600 / 12100 / 250$ & $12400 / 4200 / 100$ & $5200 / 500 / 100$ \\
\hline
\end{tabular}

${ }^{a}$ For source properties, here I refer only to the sources from the main catalogs of the $7 \mathrm{Ms}$ CDF-S (Luo et al. 2017), 2 Ms CDF-N (Xue et al. 2016), and 250 ks E-CDF-S (Xue et al. 2016). These three main catalogs were produced using essentially the same approach (critically aided by the use of the ACIS Extract package; AE; Broos et al. 2010), which incorporates a number of recent improvements in Chandra source-cataloging methodology, and therefore maximizes the number of reliable sources detected and allows for best possible X-ray characterization of source properties (see, e.g., Table 1 of Xue et al. 2016 for details).

${ }^{b}$ The E-CDF-S consists of four distinct, contiguous pointings that flank the CDF-S proper (see Fig. 2).

${ }^{c} P$ indicates the probability of a source not being real (i.e., due to background fluctuations).

${ }^{d}$ Among the $1008+683+1003=2694 \mathrm{CDF}$ sources, 298 were detected both in the CDF-S and E-CDF-S, which results in a total of 2396 unique CDF sources.

${ }^{e}$ The three numbers in parentheses denote the minimum, median, and maximum values.

$f$ This is the absorption-corrected rest-frame $0.5-7 \mathrm{keV}$ luminosity ( $L_{\mathrm{X}}$ hereafter).

${ }^{g} z_{\text {adopted }}$ denotes the adopted redshifts, with secure spectroscopic redshifts $\left(z_{\text {spec }}\right)$ preferred over photometric redshifts $\left(z_{\text {phot }}\right)$.

${ }^{h}$ The maximum secure $z_{\text {spec }}$ 's are 4.762 and 5.186 for the CDF-S/E-CDF-S and CDF-N, respectively. The $z_{\text {phot }}$ estimates above these values are subject to large uncertainties.

${ }^{i}$ These are observed source densities calculated within the respective central $r \leq 3$ arcmin areas.

of the 7 Ms CDF-S main-catalog sources have redshift measurements with $67.2 \%$ being spectroscopic ones: ${ }^{4}$ Luo et al. 2017; see Table 1). Table 2 lists some of the most notable multiwavelength surveys and photometricredshift catalogs in the CDF areas that were completed; and Figure 3 presents an example of calculating accu-

\footnotetext{
${ }^{4}$ For $z_{\text {spec }}$ references, see Section 4.3 of Luo et al. 2017 and Section 3.3.4 of Xue et al. 2016 for the CDF-S/E-CDF-S, and Section 2.3.4 of Xue et al. 2016 for the CDF-N.
}

rate $z_{\text {phot }}$ using rich multiwavelength photometric data. By combining the X-ray and multiwavelength coverage, the CDF datasets provide a unique opportunity to study both statistical and source-by-source properties of detected sources over a large range of $L_{X}-z$ parameter space (see Table 1). Furthermore, the CDF regions will be a testbed for future large observatories such as JWST and ELT, and the ultradeep multiwavelength coverage will continue to improve persistently, thereby keeping the science exciting. 
Table 2: Some Notable CDF Multiwavelength Surveys and Photometric-Redshift Catalogs

\begin{tabular}{|c|c|c|}
\hline & Surveys & $z_{\text {phot }}$ \\
\hline CDF-S/ & VLA 1.4 GHz E-CDF-S (Bonzini et al. 2012; Miller et al. 2013) & MUSYC E-CDF-S (Cardamone et al. 2010) \\
\hline \multirow[t]{18}{*}{ E-CDF-S } & ALMA LABOCA E-CDF-S (ALESS; Hodge et al. 2013) & 2 Ms CDF-S (Luo et al. 2010) \\
\hline & LABOCA E-CDF-S (LESS; Weiß et al. 2009) & E-CDF-S (Rafferty et al. 2011) \\
\hline & Herschel/HerMEs (Oliver et al. 2012) & LABOCA/E-CDF-S (Wardlow et al. 2011) \\
\hline & Herschel PEP (Lutz et al. 2011) & CANDELS/GOODS-S, CDF-S, E-CDF-S (Hsu et al. 2014) \\
\hline & GOODS-Herschel (Elbaz et al. 2011) & CANDELS/3D-HST (Skelton et al. 2014) \\
\hline & SEDS IRAC (Ashby et al. 2013) & CANDELS/GOODS-S (Santini et al. 2015) \\
\hline & SIMPLE IRAC (Damen et al. 2011) & ZFOURGE/CDF-S (Straatman et al. 2016) \\
\hline & TENIS WIRCam $K_{s}$-band (Hsieh et al. 2012) & \\
\hline & GOODS-S MUSIC (Grazian et al. 2006; Santini et al. 2009) & \\
\hline & MUSYC E-CDF-S (Taylor et al. 2009) & \\
\hline & CANDELS GOODS-S (Grogin et al. 2011; Koekemoer et al. 2011) & \\
\hline & CANDELS/3D-HST (Skelton et al. 2014) & \\
\hline & GEMS HST (Caldwell et al. 2008) & \\
\hline & COMBO-17 (Wolf et al. 2004, 2008) & \\
\hline & GOODS-S (Giavalisco et al. 2004) & \\
\hline & ESO WFI $R$-band (Giacconi et al. 2002; Giavalisco et al. 2004) & \\
\hline & HUDF (Beckwith et al. 2006) & \\
\hline & GALEX DIS (Morrissey et al. 2007) & \\
\hline \multirow[t]{13}{*}{ CDF-N } & VLA 1.4 GHz GOODS-N (Morrison et al. 2010) & CANDELS/3D-HST (Skelton et al. 2014) \\
\hline & SCUBA-2 CLS (S2CLS) GOODS-N (Geach et al. 2017) & H-HDF-N (Yang et al. 2014) \\
\hline & SCUBA HDF-N (Wang, Cowie, \& Barger 2004) & \\
\hline & Herschel/HerMEs (Oliver et al. 2012) & \\
\hline & Herschel PEP (Lutz et al. 2011) & \\
\hline & GOODS-Herschel (Elbaz et al. 2011) & \\
\hline & SEDS IRAC (Ashby et al. 2013) & \\
\hline & GOODS-N WIRCam $K_{s}$-band (Wang et al. 2010) & \\
\hline & H-HDF-N (Capak et al. 2004; Yang et al. 2014) & \\
\hline & CANDELS GOODS-N (Grogin et al. 2011; Koekemoer et al. 2011) & \\
\hline & GOODS-N (Giavalisco et al. 2004) & \\
\hline & HDF-N (Williams et al. 1996) & \\
\hline & GALEX DIS (Morrissey et al. 2007) & \\
\hline
\end{tabular}

\subsection{Identification of $X$-ray AGNs in CDFs}

Over past few decades, a series of observational and mostly empirical methods have been developed and refined to identify AGN candidates from the general X-ray source population detected in extragalactic X-ray deep surveys, including AGNs, starburst and normal galaxies, galaxy groups and clusters, and Galactic stars. Table 3 outlines the AGN identification criteria that have been routinely used in the CDF catalog papers and some relevant works (see, e.g., Bauer et al. 2004, Alexander et al. 2005a, Lehmer et al. 2008 and 2012, Xue et al. 2010, 2011, and 2016, and Luo et al. 2017 for details and caveats), which rely upon either direct use of the X-ray data or combined use of both the X-ray and multiwavelength data ${ }^{5}$ These methods are effective in revealing distinct AGN signatures owing to three major factors: accurate X-ray source positions (thanks to Chandra's sharp PSF and thus superb

\footnotetext{
${ }^{5}$ For additional methods to select AGN candidates that are undetected by X-ray observations, I refer readers to, e.g., Section 3.3 of Brandt \& Alexander (2015), for details.
}

sub-arcsecond angular resolution; see Table 1) that allow for reliable multiwavelength identifications with a sophisticated likelihood-ratio matching procedure (e.g., Brusa et al. 2005, 2007; Luo et al. 2010, 2017; Xue et al. 2016), robust X-ray photometric measurements that well describe source X-ray properties (e.g., Xue et al. 2011, 2016; Luo et al. 2017), and rich multiwavelength data that critically complement the X-ray observations (see Section 1.3 and Table 2). It is worthing noting that the vast majority of the CDF AGNs satisfy multiple criteria listed in Table 3, such cross-validations of AGN candidates therefore result in highly complete, reliable, and pure samples of distant AGNs for statistically meaningful investigations. In addition to AGN candidates, Galactic stars are also identified based on spectroscopic and/or imaging observations (e.g., Feigelson et al. 2004; Xue et al. 2011, 2016), and the remaining non-AGN and non-star CDF sources are regarded as galaxies, whose measured X-ray emission can be produced by a mixture of XRBs and low-rate SMBH accretion. 


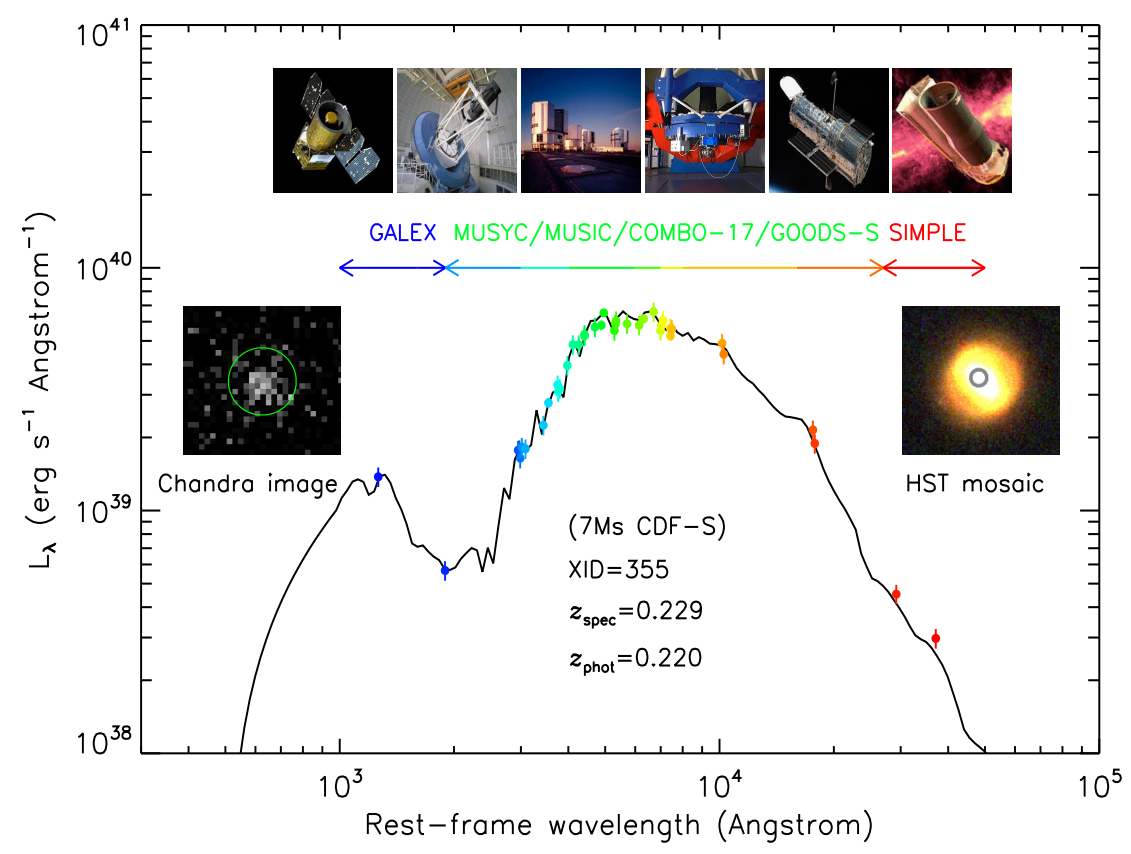

Figure 3: Reliable $z_{\text {phot }}$ calculation for the source with XID=355 in the 7 Ms CDF-S main catalog, which is enabled by using the rich and highquality broadband photometric data ( $\approx 40$ filters; see Table 2 for the detail of photometric data used) and the spectral energy distribution (SED) template fitting approach.

Table 3: Frequently-Used Criteria for X-ray AGN Identification in CDFs

\begin{tabular}{ll}
\hline \hline AGN criterion & Basic reasoning (targeted AGN subpopulation) \\
\hline$L_{\mathrm{X} \text {,intrinsic }} \geq 3 \times 10^{42} \mathrm{erg} \mathrm{s}^{-1}$ & Typical starburst and normal galaxies: $L_{\mathrm{X}, \text { intrinsic }} \lesssim 10^{42}$ erg s${ }^{-1}$ (X-ray luminous AGNs) \\
$\Gamma_{\text {effective }} \leq 1$ & Obscured $\left(N_{\mathrm{H}} \gtrsim 10^{22} \mathrm{~cm}^{-2}\right)$ AGNs: hard X-ray spectra observed (X-ray obscured AGNs) \\
$\log \left(f_{\mathrm{X}} / f_{R}\right)>-1$ & Elevated X-ray emission compared to host optical emission (majority AGN populations) \\
$\log \left(f_{\mathrm{X}} / f_{K s}\right)>-1.2$ & Elevated X-ray emission compared to host near-IR (NIR) emission (majority AGN populations) \\
$\log \left(f_{\mathrm{X}} / f_{3.6 \mu \mathrm{m}}\right)>-1.2$ & Elevated X-ray emission compared to host NIR emission (majority AGN populations) \\
$L_{\mathrm{X}, \text { intrinsic }} / L_{1.4 \mathrm{GHz}} \geq 2.4 \times 10^{18}$ & Excess X-ray emission over that expected from pure star formation (AGNs with radio data) \\
Spectroscopic AGN features & Broad emission/absorption lines; high-excitation emission lines (AGNs with quality spectra) \\
Significant X-ray variability & Large-amplitude X-ray variability commonly seen in AGNs (AGNs with $L_{\mathrm{X}, \text { intrinsic }} \geq 10^{41}$ erg s $\left.{ }^{-1}\right)$ \\
\hline
\end{tabular}

\subsection{Scope of this review}

This paper is meant to be a relatively focused and compact one, which highlights briefly some of the recent key observational results (my apology in advance for unavoidably embedding personal flavor as well as not possibly managing to cover all relevant works), mostly from the CDFs, mainly on distant AGNs, X-ray emitting galaxies, as well as galaxy groups and clusters, thus demonstrating the beauty and power of the CDF treasure trove in these relevant fields of study and hopefully arousing interest of the even broader community, in light of the recent release of the most up-todate CDF catalogs and products (Xue et al. 2016; Luo et al. 2017) yet to be fully exploited scientifically. For additional relevant in-depth reviews of results mainly from extragalactic X-ray surveys in general, I refer readers to, e.g., Comastri (2004), Gilli (2004, 2013), Brandt and Hasinger (2005), Shankar (2009), Brandt and Alexander (2010, 2015), Alexander and Hickox (2012), Fabian (2012), Treister \& Urry (2012), Kormendy \& Ho (2013), Heckman \& Best (2014), Vignali (2014), Reines \& Comastri (2016), Padovani et al. (2017), and the references therein.

The remainder of this paper is structured as follows: Section 2 describes the AGN demography results (including AGN number counts and CXRB, highredshift AGNs and AGN X-ray luminosity function XLF, highly obscured and CT AGNs, low-mass black 
holes/AGNs, and significantly variable AGNs); Section 3 highlights results on the interactions between AGNs and their host galaxies (including AGN X-ray luminosity versus galaxy SFR, conducive host galaxy properties for AGN activity, Eddington ratio distribution and correlation between intrinsic X-ray photon index and Eddington ratio, and coeval growth of SMBHs and their hosts); Section 4 introduces results on the evolution of starburst and normal galaxy X-ray emission; Section 5 presents the census of X-ray galaxy groups and clusters; Section 6 gleans some additional results not formally fit into the above sections; and finally, Section 7 summarizes this paper and discusses future prospects. Apparently, many of the above subtopics are intertwined by nature and should be understood collectively.

\section{AGN demography}

\subsection{Measuring AGN number counts and resolving the $C X R B$}

The cumulative X-ray number counts (i.e., $\log N-$ $\log S$ ), quantifying the increase of the cumulative number of X-ray sources per unit area $(N)$ as a function of decreasing flux $(S)$, has been routinely used to characterize the extragalactic X-ray source populations (e.g., Brandt et al. 2001; Rosati et al. 2002; Bauer et al. 2004; Kim et al. 2007; Georgakakis et al. 2008; Luo et al. 2008; Lehmer et al. 2012; Ehlert et al. 2013; Ranalli et al. 2013). In particular, Lehmer et al. (2012) have pushed such number-counts studies to a new level (also see, e.g., Georgakakis et al. 2008) by presenting a state-of-the-art procedure for deriving number counts in the $4 \mathrm{Ms}$ CDF-S, which performs simulations to obtain source recovery functions that account for detection incompleteness, and implements a new Bayesian approach to obtain flux-probability distributions that account for Eddington bias, thereby enabling reliable computation of number counts down to flux limits that are typically a factor of $\approx 1.5$ lower than nominal survey sensitivities.

Using the 7 Ms CDF-S main catalog and following the Lehmer et al. (2012) procedure, Luo et al. (2017; see their Fig. 31) and this paper (see Figs. 4, 5, and 6. courtesy of B. D. Lehmer) present together the unprecedentedly sensitive $\mathrm{X}$-ray number-counts measurements, down to $4.2 \times 10^{-18} \mathrm{erg} \mathrm{cm}^{-2} \mathrm{~s}^{-1}$ in the $0.5-$ $2 \mathrm{keV}$ band (soft band; SB) and $2.0 \times 10^{-17} \mathrm{erg} \mathrm{cm}^{-2} \mathrm{~s}^{-1}$ in the $2-7 \mathrm{keV}$ band (hard band; $\mathrm{HB}$; cf. the sensitivity limits shown in Table 11, for the overall source population and AGNs, respectively, confirming and/or extending significantly many previous number-counts results (e.g., Bauer etal. 2004; Lehmer et al. 2012): (1) AGNs dominate the overall number counts, and the number counts of AGNs, starburst and normal galaxies, and Galactic stars in various Chandra passbands can be adequately described by either double (for AGNs) or single power-law (for galaxies and stars) functions. (2) The galaxy power-law slope is steeper than the AGN faint-end power-law slope, indicating the rapid rise of galaxies toward faint fluxes; and, as predicted, at the faintest fluxes $\left(f_{\mathrm{SB}} \lesssim 6.0 \times 10^{-18} \mathrm{erg} \mathrm{cm}^{-2} \mathrm{~s}^{-1}\right)$ that are uniquely accessible to the $7 \mathrm{Ms}$ CDF-S, the galaxy number counts are observed for the first time to overtake the AGN number counts. (3) At the SB flux limit, the AGN and galaxy densities reach record highs of $\approx 23900$ and $\approx 26600 \mathrm{deg}^{-2}$ (the former represents the highest AGN sky density measured reliably at any wavelength; cf. the observed source densities in Table 1 , , respectively, which correspond to $\approx 1.0$ billion AGNs and $\approx 1.1$ billion X-ray galaxies in the entire sky. (4) The measurements of AGN number counts, apportioned by various source properties such as $z, N_{\mathrm{H}}$, and $L_{\mathrm{X}}$, are enabled by virtue of the exquisite CDF-S X-ray and multiwavelength data (see Table 2), which reveal that, in both SB and HB, a) AGNs with $z<1.5$ dominate the number counts while the remainder gradually catch up toward faint fluxes (see Fig. 4), and b) AGNs with $N_{\mathrm{H}}<10^{22} \mathrm{~cm}^{-2}$ or $L_{\mathrm{X}}>10^{43-44} \mathrm{erg} \mathrm{s}^{-1}$ dominate at the bright end while the others dominate or become increasingly important toward the faint end (see Figs. 5.and 6. These results are broadly consistent with the $4 \mathrm{Ms}$ CDF-S number-counts results (Lehmer et al. 2012) and the expectations of the Gilli et al. (2007) phenomenological AGN population-synthesis models. One point worth noting is that the fraction of sources with $L_{\mathrm{X}}<10^{42} \mathrm{erg} \mathrm{s}^{-1}$ that are classified as AGNs versus galaxies (see Table 3 for the AGN identification criteria) is higher in the $7 \mathrm{Ms}$ CDF-S compared to the $4 \mathrm{Ms}$ CDF-S, and this is reflected in the faint-end number counts with an upturn and also causes the galaxy number counts to not increase as fast as expected for the faintest new sources (also see, e.g., Smolčić et al. 2017a, 2017b for similar faint-end behaviors of radio AGNs).

With the number-counts estimates in hand, it is relatively straightforward to resolve the CXRB into individual X-ray sources by integrating their fluxes. Luo et al. (2017) and Lehmer et al. (2012) find that AGNs dominate the $0.5-7 / 8 \mathrm{keV}$ CXRB, and the resolved CXRB fractions are $\approx 76 \pm 4 \%$ and $82 \pm 13 \%$ with the $4 \mathrm{Ms}$ CDF-S in SB and the $2-8 \mathrm{keV}$ band (both being $\approx 1-2 \%$ higher than using the $1-2 \mathrm{Ms}$ CDFs), rising to $81 \pm 4 \%$ 

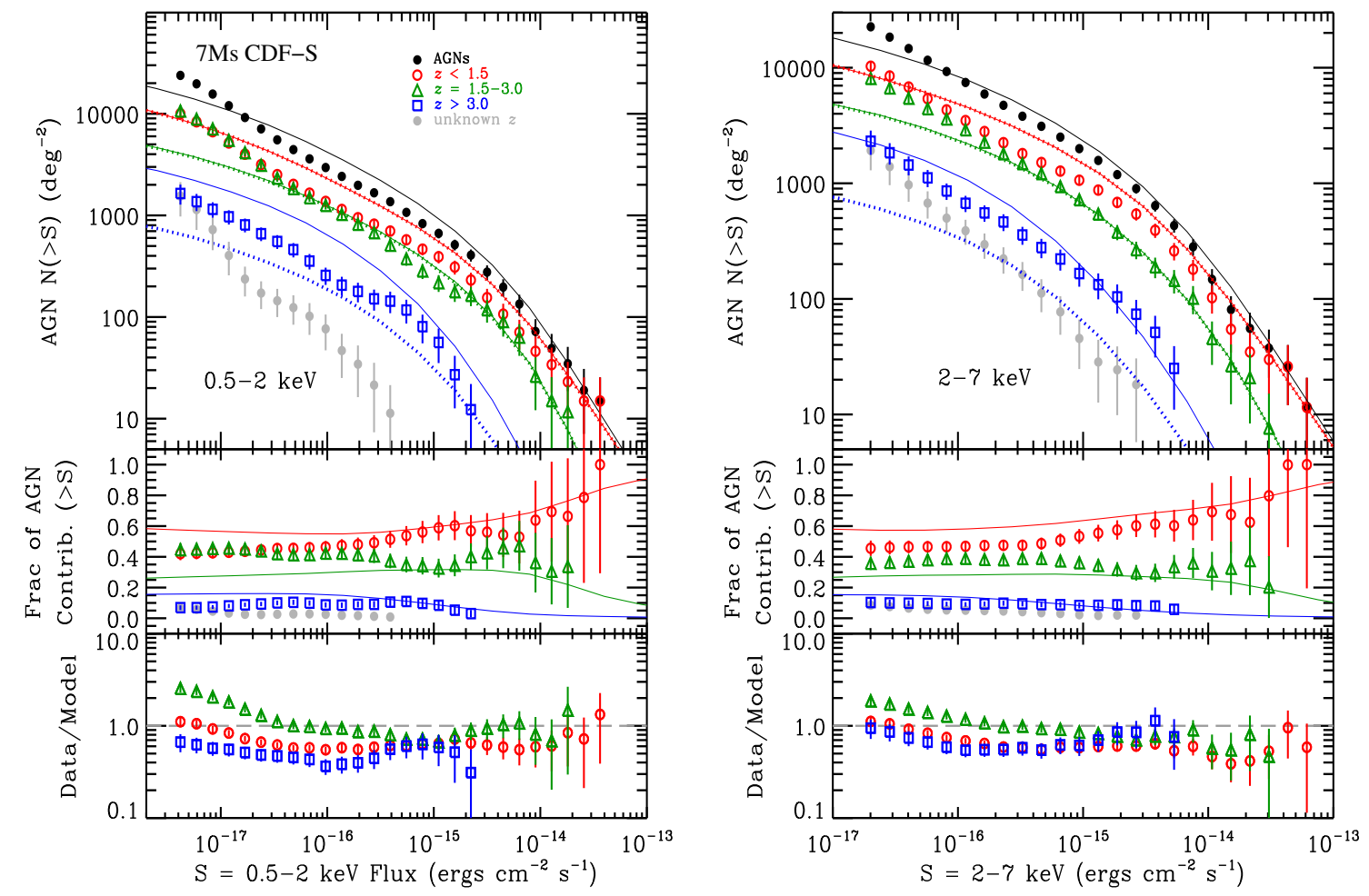

Figure 4: Top panels: cumulative AGN number counts (filled black circles) for the $7 \mathrm{Ms}$ CDF-S in the 0.5-2 keV (left) and 2-7 keV (right) bands, apportioned by AGN subsamples with $z<1.5$ (open red circles), $1.5 \leq z \leq 3.0$ (open green triangles), $z>3.0$ (open blue squares), and unknown redshifts (filled gray circles). Also shown are the Gilli et al. (2007) AGN population-synthesis model predictions (in corresponding colors) either with (dotted curves) or without (solid curves) an exponential decline in the XLF at $z>2.7$. Middle panels: fractional contribution of each AGN subsample to the overall AGN number counts. Bottom panels: the ratio between the AGN number-count data and the Gilli et al. (2007) model without a declining XLF at $z>2.7$ (courtesy of B. D. Lehmer; cf. Fig. 9 of Lehmer et al. 2012 for the 4 Ms CDF-S case).

and $93 \pm 13 \%$ with the $7 \mathrm{Ms}$ CDF-S in SB and HB, when adopting total CXRB intensities of $(8.15 \pm 0.58) \times 10^{-12}$ and $(1.49 \pm 0.20) \times 10^{-11} \mathrm{erg} \mathrm{cm}^{-2} \mathrm{~s}^{-1} \mathrm{deg}^{-2}$ in SB and HB, respectively (Hickox \& Markevitch 2006; Kim et al. 2007) ${ }^{6}$ Although it is still debated whether the resolved CXRB fraction is increasing, constant, or even decreasing toward higher bandpasses (e.g., Moretti et al. 2003; Bauer etal. 2004; Worsley etal. 2005; Georgakakis et al. 2008; Lehmer et al. 2012), the above resolved CXRB fractions are in broad agreement with many works (e.g., Bauer et al. 2004; Hickox \& Markevitch 2006; Luo et al. 2011; Xue et al. 2012), given the relatively large uncertainties on resolved fractions that primarily arise from the use of different methodologies and datasets (e.g., various AGN selection techniques,

\footnotetext{
${ }^{6}$ These resolved CXRB fractions include the bright-end corrections, which are typically $\approx 23 \%$ and $\approx 16 \%$ for the SB and $2-8 \mathrm{keV}$ band, respectively (e.g., Kim et al. 2007; Lehmer et al. 2012).
}

data depths, and redshift completeness levels) as well as CXRB intensity measurements. Despite of many efforts, the exact normalization of the CXRB spectrum is still uncertain by $\approx 20-30 \%$, due to a combination of several complicated factors that include spectral crosscalibrations, instrumental background modeling, foreground contamination by Galactic collisional thermal plasmas, stray light contamination, bright-end corrections, and cosmic variance (e.g., Bauer et al. 2004; De Luca \& Molendi 2004; Hickox \& Markevitch 2006; Kim et al. 2007; Luo et al. 2008; Moretti et al. 2009; Ishida et al. 2011; Tsujimoto et al. 2011; Lehmer et al. 2012; Cappelluti et al. 2017a; Madsen et al. 2017).

There have been some emerging works that study the CXRB from somewhat different viewpoints, either by pushing the studies of resolving the CXRB to higher energy bands due to the advent of NuSTAR (e.g., Ballan- 

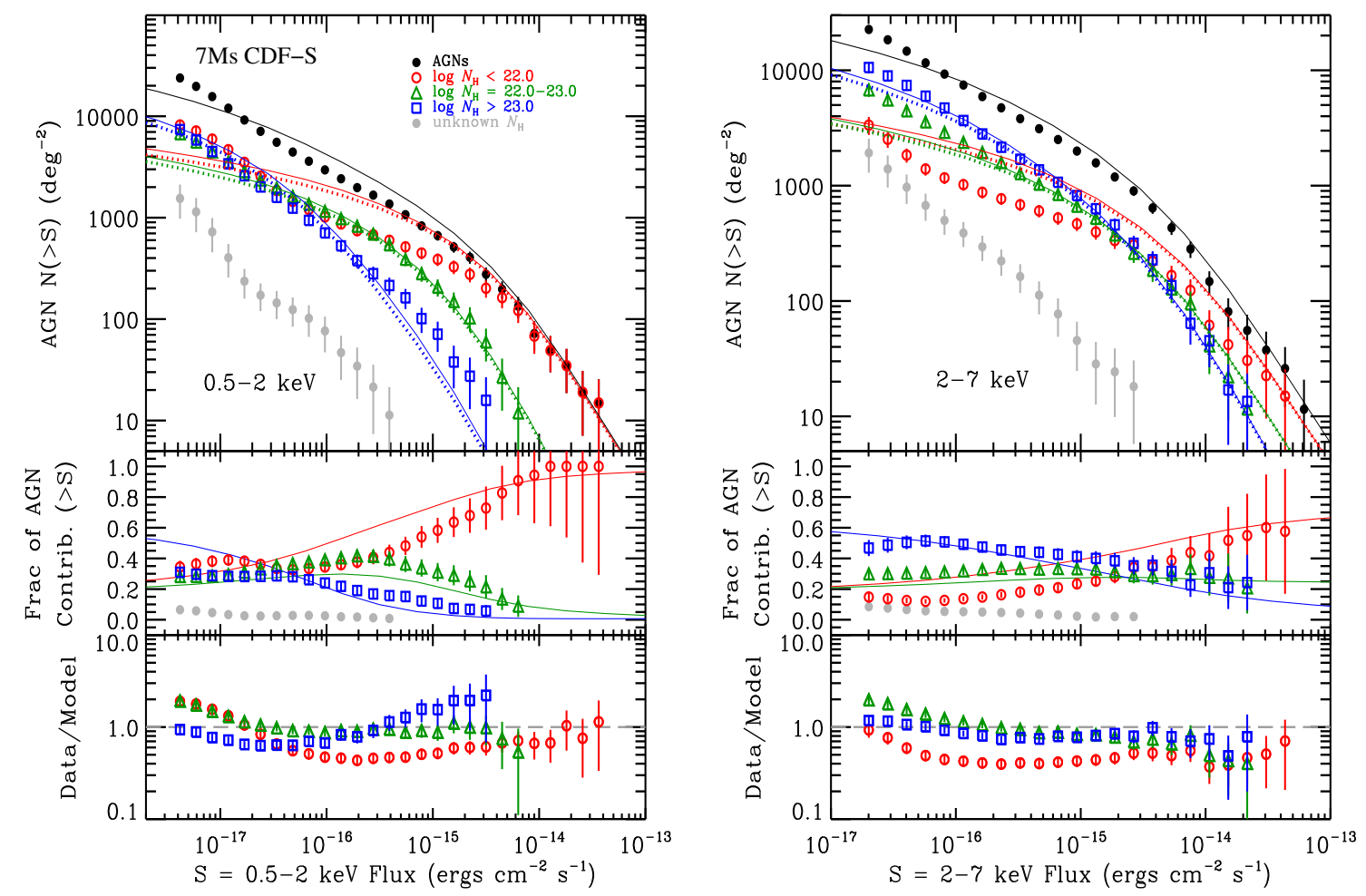

Figure 5: Same as Fig. 4 (i.e., cumulative AGN number counts for the 7 Ms CDF-S), but apportioned by AGN subsamples with different $N_{\mathrm{H}}$ values (courtesy of B. D. Lehmer; cf. Fig. 11 of Lehmer et al. 2012 for the 4 Ms CDF-S case).

tyne et al. 2011; Harrison et al. 2016) 7 or by examining the spectrum and/or origin of the unresolved CXRB (e.g., Luo et al. 2011; Moretti et al. 2012; Salvaterra et al. 2012; Xue etal. 2012; Cappelluti et al. 2017a). These studies, in conjunction with other studies, help constrain the likely existence, properties, and evolution of a missing yet potentially large population of highly obscured (i.e., $N_{\mathrm{H}} \gtrsim 3 \times 10^{23} \mathrm{~cm}^{-2}$ ) or even CT AGNs (CTAGNs hereafter; see Section 2.3), facilitating the refinement of AGN population-synthesis models (e.g., Gilli et al. 2007; Treister et al. 2009a), and revealing the various components constituting the unresolved CXRB that include likely contributions from low-luminosity AGNs (LLAGNs), highly obscured AGNs, CTAGNs, non-active galaxies, and hot gas in groups and clusters

\footnotetext{
${ }^{7}$ NuSTAR resolves $\approx 33 \%-39 \%$ of the CXRB in the $8-24 \mathrm{keV}$ band (depending on the adopted 20-50 keV CXRB intensity, and with an additional $\approx 5 \%$ statistical uncertainty), directly identifying CT AGNs with $N_{\mathrm{H}}<10^{25} \mathrm{~cm}^{-2}$ (Harrison et al. 2016). Although $\mathrm{NuS}$ $T A R$ can probe much harder (typically a factor of $\approx 3-4$ ) X-rays than Chandra and XMM-Newton, it is still not properly reaching the CXRB peak.
}

(see Section 5).

\subsection{Constraining the high-redshift AGN subpopulation and $A G N X L F$}

AGNs within the first cosmic structures at $z \approx 4-8$ and beyond are of perennial interest because their observations have serious implications for, e.g., the primordial BH seeds in the early Universe, the overall SMBH growth history (including AGN mass function and luminosity function), the origin and evolution of correlations between SMBH mass $\left(M_{\mathrm{BH}}\right)$ and galaxy properties, and the role played by AGNs during intergalactic medium (IGM) heating and reionization (see, e.g., Reines \& Comastri 2016; Brandt \& Vito 2017 for a review). Given that $\mathrm{X}$-rays are much more penetrating and much less affected by galaxy dilution (see Section 1.1) than other wavelengths, $\mathrm{X}$-ray surveys are therefore critical for assessing such high-redshift SMBH growth.

Indeed, many recent XLF works have been carried out utilizing the CDF data (note that the 7 Ms CDF-S is sufficiently sensitive to reach about $20-3$ times below 



Figure 6: Same as Fig. 4 (i.e., cumulative AGN number counts for the 7 Ms CDF-S), but apportioned by AGN subsamples with different $L_{X}$ values (courtesy of B. D. Lehmer; cf. Fig. 12 of Lehmer et al. 2012 for the 4 Ms CDF-S case).

the knee of AGN XLF at $z=0.5-4$ ) and other X-ray observations, finding that there is an exponential decline in the number density of luminous $\left(L_{\mathrm{X}} \gtrsim 10^{44} \mathrm{erg} \mathrm{s}^{-1}\right)$ AGNs with $z \gtrsim 3$ (e.g., Barger et al. 2003a; Cristiani et al. 2004; Silverman et al. 2008a; Brusa et al. 2009a; Civano et al. 2011; Fiore et al. 2012a; Vito et al. 2013, 2014a, 2017; Kalfountzou et al. 2014; Ueda et al. 2014; Georgakakis et al. 2015; Marchesi et al. 2016). However, the situation is not as clear for lower-luminosity AGNs $\left(L_{\mathrm{X}} \approx 10^{43-44} \mathrm{erg} \mathrm{s}^{-1}\right)$, largely owing to the faintness, likely heavy obscuration, survey incompleteness, limited sample size, and challenging multiwavelength follow-up of such sources. There generally also appears to be a decline in the space density of $z>3$ moderateluminosity AGNs, albeit with large uncertainties (in particular, the constraints on $z>4$ AGNs are even looser due to poorer statistics; e.g., Fiore et al. 2012a; Vito et al. 2013, 2014a, 2017; Kalfountzou et al. 2014; Ueda et al. 2014; Georgakakis etal. 2015; Marchesi et al. 2016). The space density of such AGNs remains uncertain by more than an order of magnitude (e.g., Gilli et al. 2011a), effectively having no much discriminating power between the predictions of the theoretical models that satisfy other existing observational constraints (e.g., Salvaterra et al. 2007; Marulli et al. 2008; Volonteri \& Stark 2011; Habouzit et al. 2016; Volonteri \& Reines 2016). However, even a small number of such objects, if detected, can remedy this situation and provide critical leverage in modeling early SMBH growth. In the long run, the robust detection of a decent number (i.e., a few tens or even more) of AGNs down to $L_{\mathrm{X}} \approx 10^{43} \mathrm{erg} \mathrm{s}^{-1}$ beyond $z \approx 3-4$ would provide invaluable insight on the formation mechanisms responsible for the first quasars and the early co-evolution history, which has to call for future large X-ray observatories that are capable of efficiently carrying out ultra-sensitive surveys over wide sky areas. Nevertheless, stacking analyses, instead of direct detections, have already proved to be a beneficial attempt along this direction.

Recently, Vito et al. (2016) utilize both the $7 \mathrm{Ms}$ CDF-S and ultradeep CANDELS/GOODS-S data to sensitively measure the total X-ray emission from a sample of $20763.5<z<6.5$ optically selected and individually X-ray-undetected galaxies (see Table 1 for 
the redshift-range information of individually X-raydetected sources), with a sophisticated stacking procedure that is validated by simulations. They detect highsignificance $(>3.7 \sigma)$ stacked X-ray emission from massive galaxies at $3.5<z<4.5,2.7 \sigma$ emission from those at $4.5<z<5.5$ (99.7\% confidence level; highest significance ever in such a redshift range), and no significant signal from those at $5.5<z<6.5$ (see the top panel of Fig. 7, also see, e.g., Willott 2011; Cowie, Barger \& Hasinger 2012; Fiore et al. 2012b; Basu-Zych et al. 2013). They find that the detected X-ray emission is likely dominated by high-redshift XRB populations (also see Cowie et al. 2012), by comparing it with the expected high-redshift XRB emission that is extrapolated from lower-redshift results assuming a range of XRB model prescriptions (e.g., Ranalli et al. 2003; Fragos et al. 2013a; Lehmer et al. 2016; see Section 4), and also by comparing both the total SFRs and SFR densities of the stacked galaxies with previous UV-based and SED fitting results of galaxies. Therefore, they conclude that (1) the continuous low-rate SMBH growth in individually X-ray-undetected galaxies makes negligible contribution to cosmic SMBH mass assembly at $3.5<z<6.5$, when compared to that in AGNs detected by deep X-ray surveys; and (2) the observational constraints on the faint-end $\left(L_{\mathrm{X}} \approx 10^{42} \mathrm{erg} \mathrm{s}^{-1}\right)$ AGN XLF at $3.5<z<6.5$ are achieved for the first time, which indicate a fairly flat faint-end XLF slope (see the bottom panel of Fig. 7), confirming previous results (e.g., Barger etal. 2003a; Ueda etal. 2014) and extending them down to lower X-ray luminosities.

Collectively, the AGN XLF studies over a broad stretch of redshift, including those at high redshifts that are based on direct detections and aided by the stackingbased AGN XLF constraints such as the aforementioned Vito et al. (2016) work, reveal that (1) there is an antihierachical "cosmic downsizing" behavior of AGNs, i.e., the number density of powerful quasars peaks at an earlier cosmic time than that of lower-luminosity AGNs (e.g., Cowie et al. 2003; Barger et al. 2005; Hasinger, Miyaji, \& Schmidt 2005; Silverman et al. 2008a; Yencho etal. 2009; Aird etal. 2010; Ueda etal. 2014; Miyaji et al. 2015); (2) the peak AGN emissivity (i.e., the AGN comoving bolometric luminosity density that is in units of $\mathrm{erg} \mathrm{s}^{-1} \mathrm{Mpc}^{-3}$ ) occurs at $z \approx 1.8$ for AGNs with a broad range of bolometric luminosity $L_{\mathrm{bol}}=$ $10^{43-48} \mathrm{erg} \mathrm{s}^{-1}$ (see, e.g., Fig. 20 of Ueda et al. 2014); (3) AGNs are unlikely to dominate reionization at $z \gtrsim 6$, with stars playing a leading role instead (e.g., Robertson et al. 2010, 2013; Haardt \& Madau 2012; Grissom, Ballantyne \& Wise 2014; Cappelluti et al. 2016; Vito et al. 2016; Ricci et al. 2017; also see Section 4, but see

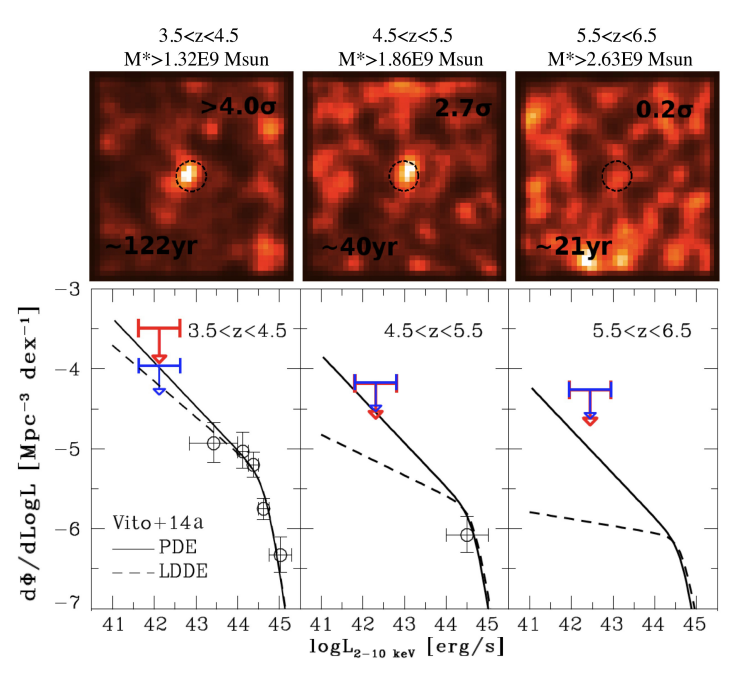

Figure 7: (Top) Smoothed stacked $40 \times 40$ pixel X-ray images (observed $\mathrm{SB}$; each image is normalized to its maximum pixel value) of optically selected and individually X-ray-undetected high-redshift galaxies in the $7 \mathrm{Ms}$ CDF-S, with various redshift bins, stellar mass ranges, stacked signal significances (e.g., the $2.7 \sigma$ emission at $4.5<$ $z<5.5$ is a detection of the highest significance ever in such a redshift range), and stacked effective exposures annotated. Dashed circles indicate the positions of the stacked galaxies. (Bottom) Upper limits on the faint-end AGN XLF for the cases of maximum AGN contribution (i.e., no SF contribution to the stacked signals; red symbols) and AGN+SF contribution (blue symbols) at high redshifts, indicating a fairly flat faint-end XLF slope at high redshifts. For comparison, the pure density evolution (PDE) and luminosity-dependent density evolution (LDDE) models and binned XLF (denoted as black open circles) presented in Vito et al. (2014a) are also shown. Adapted from Vito et al. (2016).

Giallongo et al. 2012, 2015); and (4) the luminositydependent density evolution (LDDE) and luminosity and density evolution (LADE) XLF models describe the observational data well, and the LDDE models appear to be further favored by some high-redshift constraints, yet with further testing needed due to insufficient statistics at high redshifts (e.g., Miyaji et al. 2000, 2001, 2015; Aird et al. 2010; Ueda et al. 2014; Vito et al. 2014a; Georgakakis et al. 2015; Fotopoulou et al. 2016). The basic nature of the above AGN XLF related results (e.g., cosmic downsizing) appears robust, despite of many relevant details yet to be further worked out.

\subsection{Unveiling the highly obscured and CT AGN sub- population}

Hunting for the highly obscured AGNs, CTAGNs in particular, has been a longstanding and challenging quest for the AGN demography work. CTAGNs are of special interest given a number of arguments (see, e.g., Comastri 2004; Georgantopoulos 2013; Gilli 2013; Vignali 2014 for a review): (1) There has been growing ob- 
servational evidence that a substantial fraction of AGNs are obscured by CT gas both locally (e.g., Maiolino et al. 1998; Risaliti, Maiolino, \& Salvati 1999; Matt et al. 2000) and in the distant universe (e.g., Dwelly \& Page 2006; Tozzi et al. 2006; Georgantopoulos et al. 2009; Merloni et al. 2014; Buchner et al. 2014, 2015; Lanzuisi et al. 2015a; Liu et al. 2017; also see Fig. 5). (2) The existence of the CTAGN subpopulation is required by the AGN population-synthesis models for the $\mathrm{CXRB}$ in order to account for the intensity peak of the CXRB spectrum at $\approx 20-30 \mathrm{keV}$, and CTAGNs are predicted to be as abundant as moderately obscured AGNs (e.g., Gilli et al. 2007, 2013). (3) The absorbed energy of CTAGNs at short wavelengths (including optical, UV, and X-rays) is thermally reprocessed by, e.g., the torus, and eventually reemitted in FIR, making them a potential contributor to the cosmic IR background (e.g., Shi et al. 2013a, 2013b). (4) Finally, distant CTAGNs are believed to represent a crucial phase in $\mathrm{SMBH} /$ galaxy co-evolution models, during which large amounts of gas is funneled to the center and induces both intensive obscured accretion and powerful star formation as a result of the merger process, and then feedback processes likely take over by self-regulating SMBH growth and quenching star formation (e.g., Page et al. 2004; Granato et al. 2006; Hopkins et al. 2006a; Menci et al. 2008; Alexander \& Hickox 2012). Despite their importance, the majority of distant CTAGNs escape even from the deep X-ray surveys and still remain largely elusive due to their very nature of extreme obscuration (see Fig. 8 for a demonstration); therefore, there is no good knowledge even about their space density and cosmological evolution (e.g., Aird et al. 2015a; Buchner et al. 2015). Fortunately, the situation is gradually improving as many CTAGN identification methods have been developed and many ultradeep X-ray (e.g., the CDFs) and multiwavelength surveys have been performed along the quest for CTAGNs.

The signatures of CT emission can be revealed in many ways (see Table 4 for a brief summary; some of these techniques are closely related but with different emphases), e.g.: (1) X-ray spectroscopy is the only unambiguous way to identify bona-fide CTAGNs where deep X-ray surveys play a key role. With high-quality X-ray spectra, CT $N_{\mathrm{H}}$ values can be determined reliably through careful spectral modeling, immediately unveiling the CT nature of sources (e.g., Comastri 2004; Comastri et al. 2011). (2) The presence of a strong $\mathrm{Fe} \mathrm{K} \alpha$ line complex (typically with $\mathrm{a} \gtrsim 1 \mathrm{keV}$ line equivalent width) around rest-frame $6.4-7 \mathrm{keV}$ in an AGN $\mathrm{X}$-ray spectrum is highly indicative of the source likely being CT (see Fig. 8, e.g., Comastri 2004; Comas-

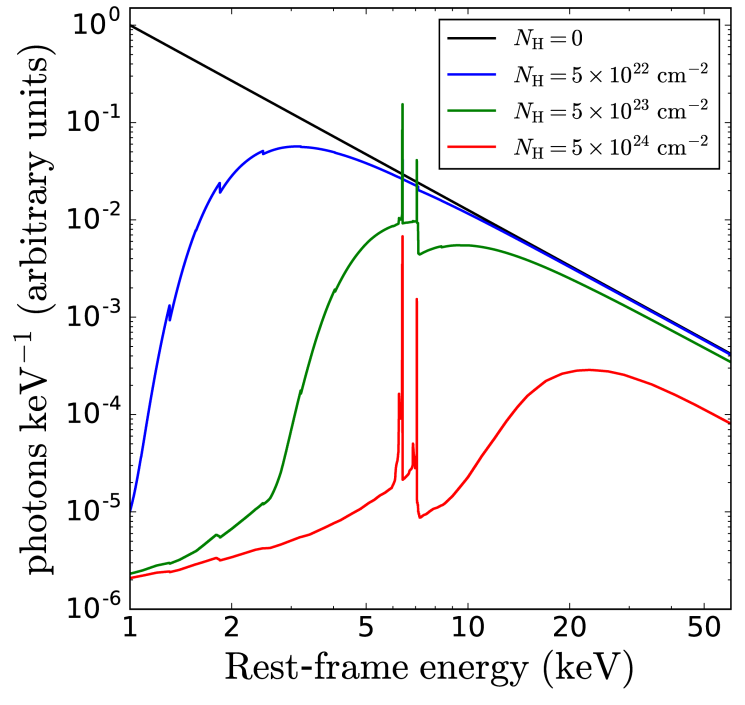

Figure 8: Model spectra of AGNs with different obscuration levels. The black, blue, green, and red curves correspond to unobscured $\left(N_{\mathrm{H}}=0\right)$, moderately obscured $\left(N_{\mathrm{H}}=5 \times 10^{22} \mathrm{~cm}^{-2}\right)$, highly obscured $\left(N_{\mathrm{H}}=5 \times 10^{23} \mathrm{~cm}^{-2}\right)$, and CT $\left(N_{\mathrm{H}}=5 \times 10^{24} \mathrm{~cm}^{-2}\right)$ AGNs, respectively. The spectra are generated using the MYTorus model with $\Gamma=1.9$, inclination angle $=90 \mathrm{deg}$, and the same normalization (Murphy \& Yaqoob 2009; courtesy of G. Yang).

tri et al. 2011; Georgantopoulos et al. 2013). (3) An AGN with a characteristic reflection-dominated X-ray spectrum is almost guaranteed to be a CTAGN; such a spectrum is featured by a broad Compton hump peaking at $\approx 20-30 \mathrm{keV}$, rapid declines toward both low energies (due to absorption) and high energies (due to Compton down-scattering), and a power-law shape in the $\approx 2-10 \mathrm{keV}$ band with a strong Fe line complex atop (typically having equivalent width $>1 \mathrm{keV}$; e.g., Comastri 2004; Comastri et al. 2011). (4) In case of an $\mathrm{X}$-ray spectrum having limited counts or not resorting to detailed spectral fitting, a (very) large X-ray hardness ratio or a (very) small $\Gamma_{\text {effective }}$ (see Table 3 can be crudely used to indicate the likely CT nature of the source (e.g., Alexander etal. 2011; Gilli et al. 2011); furthermore, using X-ray colors or a combination of $\mathrm{X}$-ray hardness and X-ray-to-MIR flux ratio can select CTAGNs more efficiently than using a single hardness ratio (e.g., Iwasawa et al. 2012; Severgnini et al. 2012). (5) An AGN having excess IR emission (owing to the reemission of the absorbed energy) compared to the typical IR/optical emission level of galaxies/nonCT AGNs are likely CTAGN candidates; the key to this category of "IR-excess" methods is to reduce contamination from starburst and normal galaxies (see, e.g., Daddi et al. 2007, Alexander et al. 2011, and Luo et al. 
Table 4: Techniques to Identify CTAGN Candidates

(1) $\mathrm{CT} N_{\mathrm{H}}$ values derived from X-ray spectral fitting

(2) Strong Fe $\mathrm{K} \alpha$ line complex

(3) Characteristic X-ray reflection spectrum/component

(4) Large X-ray hardness ratio + X-ray colors

(5) IR-excess emission

(6) X-ray stacking

(7) Some well-calibrated luminosity ratios

(8) Deep $9.7 \mu \mathrm{m}$ Si feature with $>1$ optical depth

(9) Spectral curvature above $10 \mathrm{keV}$

(10) Broadband SED decomposition

See main text for explanations and references therein for caveats.

2011 for a SFR-excess method; and Sacchi et al. 2009 for a high MIR/optical flux-ratio method). (6) X-ray stacking takes benefit of Chandra's superb angular resolution, thus allowing for exploring considerably deeper $\mathrm{X}$-ray fluxes and revealing the average properties of the stacked sample of CTAGN candidates (e.g., Luo et al. 2011; Xue et al. 2012). (7) Some well-calibrated luminosity ratios appear to have good discriminating power between $\mathrm{CT}$ and non-CT AGNs, e.g.: luminosity ratios between X-ray and high-ionization emission lines from the narrow-line region (thus not suffering from

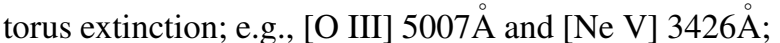
e.g., Vignali etal. 2006; Ptak et al. 2006; Gilli et al. 2010; Mignoli et al. 2013), and luminosity ratios between X-ray and 6/8/12 $\mu \mathrm{m}$ band (e.g., Lutz et al. 2004; Fiore et al. 2009; Alexander et al. 2011; Georgantopoulos et al. 2011b; Mateos et al. 2015). (8) An AGN with a very deep Si feature at $9.7 \mu \mathrm{m}$ that has an optical depth of $\tau>1$ (i.e., $\tau_{9.7 \mu \mathrm{m}}>1$ ) in its IR spectrum is very likely to be a CTAGN (e.g., Georgantopoulos et al. 2011a). (9) A newly-developed metric that utilizes the $>10 \mathrm{keV}$ spectral curvature to identify CTAGNs with Swift/NuSTAR data proves to be quite effective (Koss et al. 2016). (10) If the broadband SED decomposition requires a strong AGN torus emission, this can then serve as a supporting evidence of the source likely being CT (e.g., Lanzuisi et al. 2015b; Del Moro et al. 2013, 2016). Among various selection methods for CTAGNs, X-rays represent a fundamental and powerful probe through direct X-ray spectroscopy and reliable stacking analysis. Whenever data allow, it would be beneficial to cross validate the identified CTAGN candidates using as many methods as possible and meanwhile keep in mind the biases and limitations (e.g., incompleteness and contamination) associated with each method (see the above relevant references for details).
There have been many works that involve the use of one or more of the above methods and prove their utility. For instance, Gilli et al. (2011) discover a $z_{\text {spec }}=4.762$ CTAGN in the 4 Ms CDF-S that is confirmed by X-ray spectral analysis, thus being the most distant bona-fide CTAGN so far. This source appears to be caught during a major coeval episode of SMBH accretion $\left(L_{2-10 \mathrm{keV}} \approx 2.5 \times 10^{44} \mathrm{erg} \mathrm{s}^{-1}\right)$ and stellar mass assembly (being a submillimeter galaxy (SMG) with $\mathrm{SFR} \approx 1000 M_{\odot} \mathrm{yr}^{-1}$ that is later confirmed by ALMA observations; Coppin et al. 2009; Gilli et al. 2014) at early times (see Fig 9). More interestingly, several lines of evidence point to the existence of outflowing winds from the central SMBH, hinting at AGN feedback at work (Gilli et al. 2014). Finding more such AGNs and constraining their number density would be crucial to reconstruct the early co-evolution history. Alexander etal. (2011) identify 11 highly obscured AGNs among a sample of $z \approx 2 K<22 \mathrm{mag} B z K$-selected galaxies through X-ray spectral analysis in the $4 \mathrm{Ms}$ CDF-S. They find that, among these 11 sources, some display excess IR emission and some prefer a pure reflection model; furthermore, the stacked X-ray spectrum of all the sources has a reflection-dominated shape and a strong Fe line feature, indicating CT absorption in some sources (see the left panel of Fig. 10; ; and the estimated lower-limit space-density constraints of highlyobscured AGNs/CTAGNs (i.e., $\geq f \times 4 \times 10^{-5} \mathrm{Mpc}^{-3}$ for CTAGNs with $L_{2-10 \mathrm{kev}} \gtrsim 10^{43} \mathrm{erg} \mathrm{s}^{-1}$ and $z \approx 2$, where $f \approx 10-50 \%$ is the likely CTAGN fraction within the studied sample) are already comparable to the results from some other works (e.g., Tozzi et al. 2006; Alexander et al. 2008; Fiore et al. 2009; Brightman \& Ueda 2012; Del Moro et al. 2016) and the predictions from AGN population-synthesis models, albeit with large uncertainties on both observations (see the right panel of Fig. 10) and models (e.g., Gilli et al. 2007; Treister et al. 2009a). It appears that the space density of $z \approx 2$ CTAGNs is comparable to that of $z \approx 2$ unobscured AGNs, suggesting a non-negligible contribution of such CTAGNs to the overall SMBH growth at $z \approx 2$.

Most recently, Liu etal. (2017) present a systematic spectral analysis for a sample of $276 \mathrm{HB}$-selected brightest AGNs in the $7 \mathrm{Ms}$ CDF-S. After correcting for sample selection biases (e.g., incompleteness and Eddington bias), they find that: (1) The intrinsic $N_{\mathrm{H}}$ distribution varies significantly across different redshift ranges (up to $z \approx 5$ ) as a result of strong dependence of $N_{\mathrm{H}}$ on both $L_{\mathrm{X}}$ and $z$; the overall $N_{\mathrm{H}}$ distribution peaks at $N_{\mathrm{H}} \approx 10^{23.5-24} \mathrm{~cm}^{-2}$, higher than the peak value of $N_{\mathrm{H}} \approx 10^{23.1} \mathrm{~cm}^{-2}$ derived with $1 \mathrm{Ms}$ 


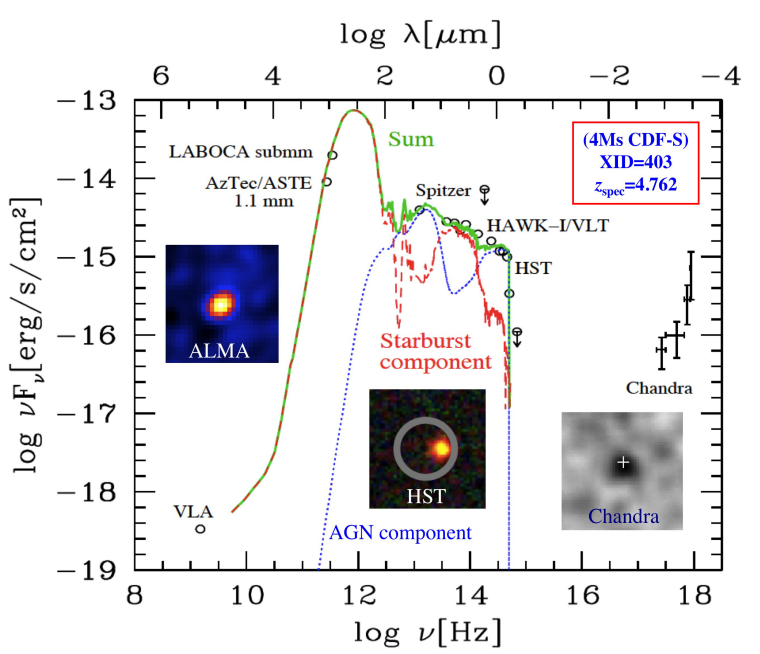

Figure 9: Broadband observed SED of a $z_{\mathrm{spec}}=4.762 \mathrm{CTAGN}$ (i.e., the most distant bona-fide CTAGN discovered so far that has $\mathrm{XID}=403$ in the $4 \mathrm{Ms}$ CDF-S) and its possible decomposition into a galaxy component (i.e., shifted Arp 220 template; red dashed curve) and an AGN component (i.e., sum of a torus and a scattered component; blue dotted curve) with their sum shown as the green solid curve. The inset ALMA image is from Gilli et al. (2014). Adapted from Gilli et al. (2011b).

CDF-S (Tozzi et al. 2006; also see, e.g., Burlon et al. 2011; Brightman \& Nandra 2011; Buchner et al. 2015), mainly due to more highly obscured sources being revealed by the 7 Ms CDF-S. (2) The strong evolution of obscured AGN fraction with $z$ can be formulated as $f_{\text {obscured }}=(0.43 \pm 0.07)(1+z)^{0.59 \pm 0.12}$; at $z>2$, $f_{\text {obscured }}$ shows only a weak evolution, likely being saturated (see also, e.g., Hasinger 2008; Vito et al. 2014a; Ueda et al. 2014); and the $f_{\text {obscured }}$ measures are higher than previous ones (e.g., Iwasawa et al. 2012; Vito et al. 2014a). (3) After combining the 7 Ms CDF-S data with the wider and shallower C-COSMOS data (Lanzuisi et al. 2013), it is clear that, at any given (small) redshift bin within the range of $0.3<z<4.0$, the average $N_{\mathrm{H}}$ (or $f_{\text {obscured }}$ ) decreases with $L_{\mathrm{X}}$; and at any given (small) $L_{X}$ bin within the range of $42<\log \left(L_{X}\right)<45$, the average $N_{\mathrm{H}}$ (or $f_{\text {obscured }}$ ) increases with $z$. (4) A total of 22 CTAGNs are identified, corresponding to $\approx 8 \%$ of the studied sample; such a CTAGN fraction appears comparable to or slightly lower than CTAGN fractions of $\approx 7-20 \%$ among hard X-ray selected samples (e.g., Malizia et al. 2009; Burlon et al. 2011; Vasudevan et al. 2013; Akylas etal. 2016). The above dependence of $f_{\text {obscured }}$ on $L_{\mathrm{X}}$ result presented by Liu et al. (2017) confirms many previous works (Treister and Urry 2006; Hasinger 2008; Brightman \& Nandra 2011; Burlon et al. 2011; Lusso et al. 2013; Brightman et al. 2014; Merloni et al. 2014; Ueda et al. 2014) and extends reliably to lower $L_{\mathrm{X}}$. Surprisingly, wide-field IR surveys find that $f_{\text {obscured }}$ might rise substantially again at $L_{\text {bol }} \approx 10^{47} \mathrm{erg} \mathrm{s}^{-1}$ (e.g., Stern et al. 2014; Assef et al. 2015). Moreover, the dependence of $f_{\text {obscured }}$ on $z$ result given by Liu et al. (2017) also confirms many previous works (e.g., La Franca et al. 2005; Ballantyne et al. 2006; Tozzi et al. 2006; Treister and Urry 2006; Hasinger 2008; Treister et al. 2009a; Hiroi et al. 2012; Iwasawa et al. 2012; Vito et al. 2013, 2014a; Brightman et al. 2014; Merloni et al. 2014; Ueda et al. 2014; Buchner et al. 2015) and extends reliably to lower $L_{\mathrm{X}}$.

An improved demography picture for the highly obscured and CT AGNs that better reveals their space density and cosmological evolution can be obtained, by combining and reconciling all the bits and pieces of works already present in the literature, which are based on direct X-ray spectroscopy, reliable X-ray stacking analysis, and/or various multiwavelength diagnostics (e.g., Daddi et al. 2007; Alexander et al. 2008a, 2011; Fiore et al. 2008, 2009; Treister et al. 2009b; Gilli et al. 2011; Luo et al. 2011; Brightman \& Ueda 2012; Vignali et al. 2014; Del Moro et al. 2016), as well as $N u S$ TAR's > $10 \mathrm{keV}$ constraints (e.g., Alexander et al. 2013; Del Moro et al. 2014; Brightman et al. 2015; Koss et al. 2016). However, there is still a long way to a complete understanding of the CTAGN demography given the currently small sample of distant bona-fide CTAGNs; and the radical change of such a situation would have to rely on new powerful and preferably hard X-ray facilities.

Last but not least, accumulating evidence shows that the AGN obscuring material may not be necessarily related to the pc-scale dusty torus as commonly accepted; instead, it may be attributed to a compact starburst region (e.g., Gilli et al. 2014), the diffuse interstellar medium in the host galaxy (with $N_{\mathrm{H}}$ likely up to $10^{22-23.5} \mathrm{~cm}^{-2}$; e.g., Simcoe etal. 1997; Goulding et al. 2012; Buchner \& Bauer 2017; Lanzuisi et al. 2017), or 100 pc-scale dust filaments (e.g., Prieto et al. 2014) 8 Such obscuring material existing at relatively large scales likely has multiple functions, e.g., fueling AGN accretion, igniting nuclear star formation, and exerting AGN feedback (e.g., in the form of AGNdriven outflows) to influence the host galaxy, which adds an additional complexity to the understanding of

\footnotetext{
${ }^{8}$ Most recently, Maiolino et al. (2017) find observational evidence for star formation $\left(\mathrm{SFR} \approx 15 M_{\odot} \mathrm{yr}^{-1}\right.$ ) occurring within a prominent galactic outflow, which is $\approx 7-9 \mathrm{kpc}$ away from and perhaps driven by the southern obscured AGN in a $z=0.0448$ merging system, likely playing some role in obscuring the AGN.
} 

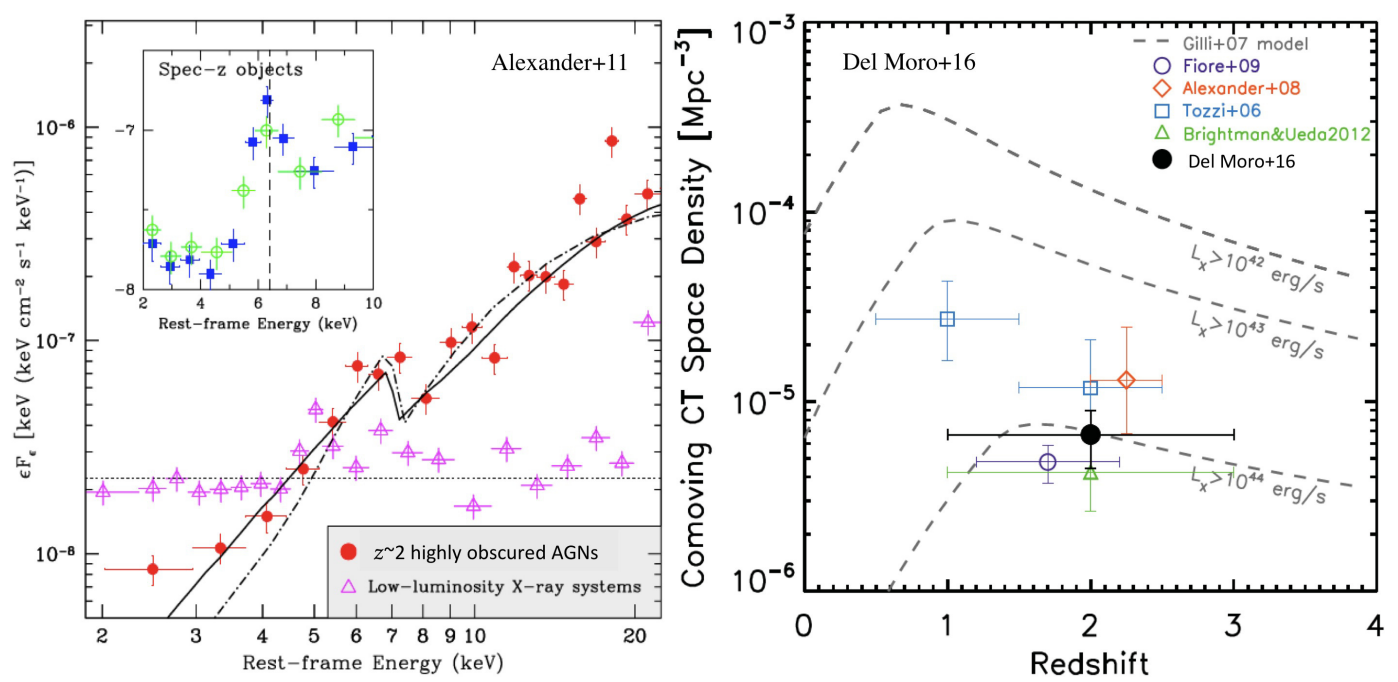

Figure 10: (Left) Composite rest-frame 2-20 keV spectra for the $z \approx 2$ highly obscured AGNs (filled circles) and low-luminosity X-ray sources (open triangles) as compared to an unabsorbed power-law model (dotted line; intrinsic photon index $\Gamma=2.0$ ), a pure reflection model (solid curve; $\Gamma=1.7$ ), and a best-fit model to a reflection-dominated local AGN (dot-dashed curve). The inset highlights the stacked X-ray spectra of the highly obscured AGNs with $z_{\text {spec }}$ (filled squares: all sources; open circles: all sources except one with an individually identified Fe K $\alpha$ line). Several lines of evidence points to the existence of CTAGNs in the studied sample (see Table 4). (Right) Comoving space densities of CT quasars/AGNs from Del Moro et al. (2016; black filled circle), Brightman \& Ueda (2012; green open triangle), Fiore et al. (2009; purple open circle), Alexander et al. (2008a; orange open diamond), and Tozzi et al. (2006; blue open squares), in comparison with the Gilli et al. (2007) AGN population-synthesis model predictions for AGNs with various X-ray luminosities. (Left) adapted from Alexander et al. (2011) and (Right) adapted from Del Moro et al. (2016).

the AGN obscuration phenomenon and makes the quest for highly obscured/CT AGNs even more challenging yet more intriguing.

\subsection{Searching for the low-mass BH/AGN subpopula- tion}

Low-mass BHs (or intermediate-mass BHs; IMBHs: 9 ? with $\approx 10^{3-6} M_{\odot}$ ) are thought to naturally bridge the evolutionary gap between stellar-mass BHs (with a few tens of solar masses) and SMBHs, whose studies have important implications for the origin of SMBHs (i.e., the birth and growth of early BH seeds), the overall SMBH growth history, the common characteristics of $\mathrm{BH}$ phenomena across all mass scales, and many other aspects (see, e.g., Miller \& Colbert 2004; Volonteri 2010; Greene 2012; Reines \& Comastri 2016 for a review). However, such a population of low-mass BHs has been largely elusive, because they are difficult to find, mainly due to their low masses and thus small gravitational influences and low luminosities.

Nevertheless, the situation of searching for low-mass BHs has been improving continuously, thanks to the

\footnotetext{
${ }^{9} \mathrm{~A}$ most recent work, which discovers an IMBH with $2200_{-800}^{+1500} M_{\odot}$ in the center of the globular cluster 47 Tucanae, has become a real eye catcher (Kiziltan, Baumgardt, \& Loeb 2017).
}

advent of numerous high-quality multiwavelength observations and various search techniques. Within the Local Group, it is possible to utilize stellar or gas dynamics to search for low-mass BHs residing in dwarf galaxies (less than a few $10^{9} M_{\odot}$ ), which represent a plausible place to host such BHs according to, e.g., the $M_{\mathrm{BH}}-M_{\star}$ scaling relation (see, e.g., Kormendy \& Ho 2013 for a review). A number of such attempts made in some local dwarf galaxies either place upper limits of $M_{\mathrm{BH}} \lesssim 10^{4} M_{\odot}$ (e.g., Gebhardt et al. 2001; Valluri et al. 2005; Lora et al. 2009; Jardel \& Gebhardt 2012) or find some positive measurements of $M_{\mathrm{BH}} \approx 10^{5-6} M_{\odot}$ (e.g., Seth et al. 2010; van den Bosch \& de Zeeuw 2010; den Brok et al. 2015). Going beyond the Local Group, multiwavelength techniques have to be invoked in order to reveal the signatures of low-mass AGNs typically located in dwarf galaxies. For example, optical spectroscopic features such as high-ionization narrow emission lines, broad emission lines, and narrow emissionline diagnostics reveal many samples of low-mass BHs or candidates with $M_{\mathrm{BH}} \approx 10^{5-6} M_{\odot}$ (e.g., Greene \& Ho 2004; Dong et al. 2012; Reines et al. 2013; Moran et al. 2014; Sartori etal. 2015); and some studies attempt to use MIR color diagnostics to search for lowmass AGNs in small-bulge and low-mass galaxies (e.g., 
Satyapal et al. 2014; Hainline et al. 2016). It should be noted that the above optical techniques tend to be biased toward AGNs with relatively high Eddington ratios ( $\lambda_{\mathrm{Edd}}=L_{\mathrm{bol}} / L_{\mathrm{Edd}}$, where $L_{\mathrm{Edd}}$ is Eddington luminosity) and MIR approaches are often subject to severe contamination because of the confusion between MIR colors of dwarf starburst galaxies and AGNs. In contrast, X-ray observations do not have such limitations and can probe AGNs down to very low levels with least bias (see Section 1.1.

Indeed, many X-ray studies have accumulated growing samples of low-mass AGNs among a wide variety of low-mass host galaxies mostly in the local universe (e.g., Ghosh et al. 2008; Jia et al. 2011; Reines et al. 2011, 2014; Kamizasa, Terashima, \& Awaki 2012; Lemons et al. 2015; Miller et al. 2015; Baldassare et al. 2015, 2017; Plotkin et al. 2016). The search for lowmass AGNs at moderate redshifts calls for deep X-ray surveys with direct detections or stacking analyses. Schramm et al. (2013) discover three low-mass AGNs hosted in $M_{\star}<3 \times 10^{9} M_{\odot}$ galaxies at $z<0.3$, which are individually detected in the $4 \mathrm{Ms}$ CDF-S and have $L_{\mathrm{X}} \approx 10^{40} \mathrm{erg} \mathrm{s}^{-1}$ (with one being variable). Particularly, one of these sources has its broad $\mathrm{H} \alpha$ line detected, providing a virial mass estimate of $\approx 2 \times 10^{5} M_{\odot}$ that is consistent with the estimate derived using scaling relations between $M_{\mathrm{BH}}$ and host galaxy properties (see Fig. 11). Pardo et al. (2016) identify 10 low-mass X-ray AGNs among a sample of $M_{\star}<3 \times 10^{9} M_{\odot}$ dwarf galaxies at $z<1$, which are estimated to typically have $\lambda_{\text {Edd }} \approx 5 \%$. Most recently, Chen et al. (2017) study a sample of 10 low-mass $z<0.3$ AGNs detected in the 3-24 keV band by the NUSTAR serendipitous survey, demonstrating the great potential and advantage of using hard X-ray observations to recover low-mass (obscured) AGNs missed by optical spectroscopic surveys and $<10 \mathrm{keV} \mathrm{X}$-ray surveys. Furthermore, the use of sensitive stacking can significantly push the constraints upon low-mass AGNs out to higher redshifts. By stacking the 4 Ms CDF-S data, Xue et al. (2012) track down a population of highly obscured AGNs that are not individually detected in X-rays, which make the majority contribution to the unresolved $6-8 \mathrm{keV}$ $\mathrm{CXRB}$, and are hosted in faint $1 \lesssim z \lesssim 3$ galaxies that are located on the top of the blue cloud and has $M_{\star} \approx 2 \times 10^{8-9} M_{\odot}$. Mezcua et al. (2016) stack a large sample of X-ray-undetected dwarf starburst and latetype galaxies in five redshift bins up to $z=1.5$ using the Chandra COSMOS-Legacy survey data, and find AGN $\mathrm{X}$-ray emission (with mean $L_{\mathrm{SB}} \approx 10^{39-40} \mathrm{erg} \mathrm{s}^{-1}$ ) in each redshift bin after removing contributions of XRB and hot gas to the stacked signal. It seems promising

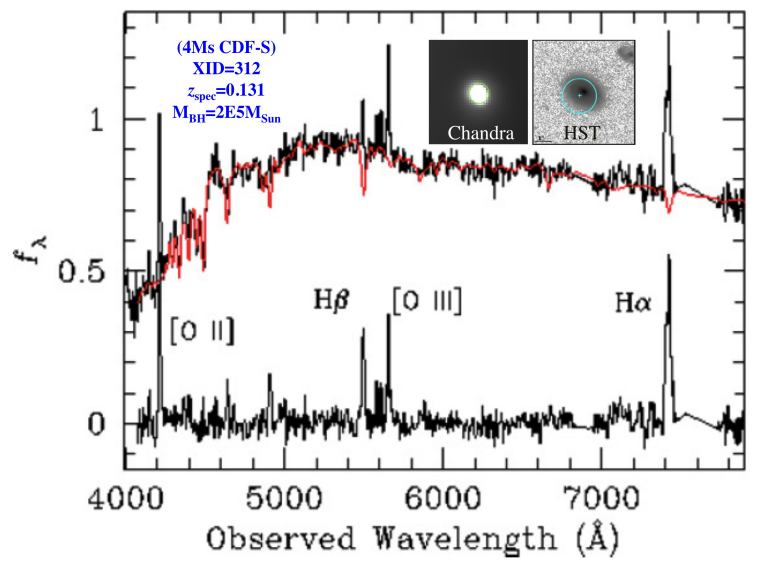

Figure 11: An example of low-mass AGNs: VLT/FORS2 optical spectra (before and after stellar continuum subtraction; given in relative flux) of a $z_{\text {spec }}=0.131$ source $(\mathrm{XID}=312$ in the $4 \mathrm{Ms} \mathrm{CDF}-\mathrm{S}$ ) that has an estimated $M_{\mathrm{BH}}=2 \times 10^{5} M_{\odot}$ based on the virial mass estimate using the broad $\mathrm{H}_{\alpha}$ line. The red curve indicates the best-fit continuum. Adapted from Schramm et al. (2013).

for subsequent studies to obtain a key advance by finding better multiwavelength tracers of which galaxies are those likely to host AGNs, which can allow much improved X-ray stacking and diagnosis of physical drivers.

Combining the efforts of searching for low-mass AGNs with optical, MIR, and particularly X-ray observations, it appears evident that low-mass AGNs are quite common from the local universe at least out to moderate redshifts. Therefore, it is imperative to further build up statistically meaningful samples of lowmass AGNs across a reasonably broad redshift range and with a variety of host properties, which will certainly help us piece together a scenario for their evolution, lend lessons to studies of high-redshift SMBH seeds, make a critical step forward in understanding the AGN demography in the low-BH mass regime, and constrain further their behavior in the scaling relations between $M_{\mathrm{BH}}$ and host galaxy properties (e.g., Greene et al. 2010; Jiang, Greene, \& Ho 2011; Graham \& Scott 2013, 2015; Reines \& Volonteri 2015; Baldassare et al. 2015; Ho \& Kim 2016).

\subsection{Examining the significantly variable AGN subpop- ulation}

Temporal and spectral variability is a defining and ubiquitous feature of AGNs, and variability studies are valuable for probing AGN physical properties (e.g., Mushotzky et al. 1993; Ulrich etal. 1997; Peterson 2001; Vaughan etal. 2003). The versatile utility of AGN variability analyses is rendered in many aspects, 
e.g.: (1) A first-order estimation of the physical size of the emission region can be obtained by searching for the minimum variability timescale according to simple light-crossing time arguments, which is often employed in high-energy (X-ray and gamma-ray) observations of blazars (e.g., Xue \& Cui 2005; Abdo et al. 2011). (2) Detailed reverberation-mapping based studies allow for size estimates of various AGN components such as accretion disk, broad-line region, and inner part of dusty torus (e.g., Koshida et al. 2014; Peterson 2014; Fausnaugh et al. 2016). (3) The properties of the absorbing matter such as obscuring wind and gas can be revealed by examining the changes in absorption (e.g., Filiz Ak et al. 2013; Netzer 2015). (4) The AGN X-ray power spectral density (PSD) is often modeled with a broken power-law of red-noise nature (e.g., Uttley, McHardy, \& Papadakis 2002; also see Fig. 1 of Zhu \& Xue 2016 for a demonstration). The break frequency of the PSD, the amplitude of the high-frequency PSD, and the integral of that part are found to be closely related to $\mathrm{BH}$ properties such as $M_{\mathrm{BH}}$ and mass accretion rate, thereby enabling measurements of such $\mathrm{BH}$ properties (e.g., McHardy etal. 2004, 2013; Zhou et al. 2010; González-Martín \& Vaughan 2012; Kelly et al. 2013). (5) Significant variability has often been used as an effective AGN selection technique in X-ray, UV, and optical bands (e.g., Maoz et al. 2005; Trevese et al. 2008; Boutsia et al. 2009; Villforth et al. 2010; MacLeod et al. 2011; Young et al. 2012; Falocco et al. 2015; also see Table 3. (6) AGN variability observations can place constraints on the underlying emission processes as well as the models intended to explain the physical origins of AGN variability (e.g., Xue, Yuan, \& Cui 2006; Kelly et al. 2009, 2014; Cai et al. 2016; Liu et al. 2016a).

AGN X-ray variability is of particular interest, because it is generally more rapid and has larger amplitudes than variability at longer wavelengths; moreover, $\mathrm{X}$-ray emission is nearly universal among AGNs (see Section 1.1) and it originates from the innermost part of the system, thus being able to probe the immediate vicinity of the SMBH (e.g., Ulrich et al. 1997). Therefore, many deep X-ray surveys have been conducted to study AGN variability down to low and moderate luminosities and up to high redshifts (e.g., Almaini et al. 2000; Paolillo et al. 2004, 2017; Mateos et al. 2007; Papadakis et al. 2008; Vagnetti et al. 2011, 2016; Lanzuisi et al. 2014; Shemmer et al. 2014; Middei et al. 2017; Zheng et al. 2017; Li et al. in prep.). The observations of the CDFs, particularly the CDF-S and CDF-N, cover a timespan up to $\approx 16$ years (see Table 1 ), resulting in the longest rest-frame timescales that can possibly be probed for X-ray variability analyses of a large sample of distant AGNs, and thus enabling a range of exciting variability science.

Young etal. (2012) identify 20 AGNs out of a sample of 92 galaxies with $z \approx 0.08-1.02$ in the $4 \mathrm{Ms}$ CDF-S, solely based on their significant long-term X-ray variability. These 20 newly-identified AGNs fail all the other non-variability AGN identification criteria adopted by the $4 \mathrm{Ms}$ CDF-S cataloging work (Xue et al. 2011; also see Table 3) and were then regarded as galaxies. These 20 AGNs have observed variability behavior that cannot be explained by XRB populations, and appear to be low-luminosity unobscured AGNs given their stacked $\Gamma_{\text {effective }}=1.93 \pm 0.13$, with estimated $M_{\mathrm{BH}}$ (accretion rates) a factor of 2.4 (22.5) lower than variable luminous AGNs at the same redshift. This study underscores the advantage of using X-ray variability analysis to find low-luminosity unobscured AGNs that would otherwise be missed. Yang et al. (2016) conduct systematic long-term $\mathrm{X}$-ray variability analyses of the 68 brightest radio-quiet AGNs at $z \approx 0.6-3.1$ in the $6 \mathrm{Ms}$ CDF-S and find: (1) Among these sources, $\approx 90 \%$ are variable, indicating widespread photon flux variability; $\approx 74 \%$ display $L_{\mathrm{X}}$ variability, with quasars having smaller variability amplitudes; and $\approx 16 \%$ show $N_{\mathrm{H}}$ variability, with variability amplitudes becoming larger for longer time separation. (2) There are a few sources possessing very interesting variability patterns: a CTAGN candidate has variable high-energy X-ray flux, implying the existence of $\lesssim 0.3$ pc reflecting matter; a broad absorption line quasar is $L_{\mathrm{X}}$ variable; and a $z_{\text {spec }}=1.21$ source appears to be a "semi-changinglook" AGN, transiting from an X-ray unobscured to obscured state (also see, e.g., Matt, Guainazzi, \& Maiolino 2003; Ricci et al. 2016 for examples of X-ray changinglook AGNs) but always remaining as optically type I (see Fig. 12, also see, e.g., LaMassa et al. 2015; McElroy et al. 2016; Runnoe et al. 2016 for examples of optical changing-look AGNs). This work showcases the uniqueness of the CDF-S dataset in revealing long-term AGN variability and some unusual variable AGNs. Paolillo et al. (2017) apply various PSD models to study the ensemble X-ray timing properties of AGNs during a timespan of $>16$ years up to $z \approx 4$ in the $7 \mathrm{Ms} C D F-S$, and find that the average $\lambda_{\text {Edd }}$ appears to be largely constant across this redshift range given the large statistical uncertainties, with only marginal evidence for a possible increase of $\lambda_{\text {Edd }}$ that peaks at $z \approx 2-3$. This study shows the potential of using X-ray variability analysis to trace the overall SMBH accretion history, which can be fully developed when future large X-ray missions enlarge AGN samples and thus reduce statistical uncertainties associated with such measurements. 

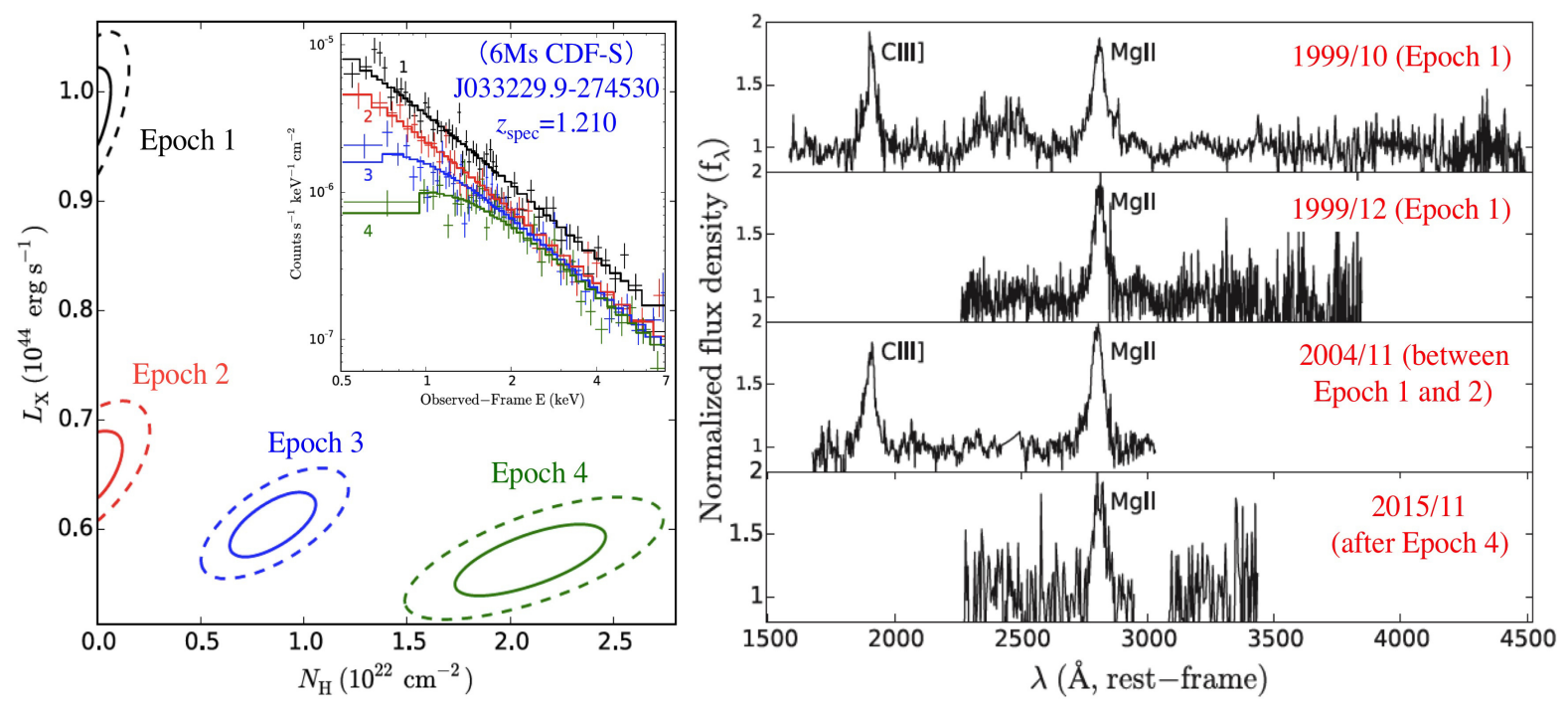

Figure 12: (Left) Unfolded X-ray spectra, best-fit models (data binned for display purposes only; inset) and $L_{\mathrm{X}}-N_{\mathrm{H}}$ confidence contours (1 $\sigma$ and $2 \sigma$ ) for J033229.9-274530 in the 6 Ms CDF-S during four epochs (Epoch 1: 1999/10-2000/12; Epoch 2: 2007/09-2007/11; Epoch 3: 2010/032010/07; Epoch 4: 2014/06-2015/01), which displays significant $N_{\mathrm{H}}$ variability. (Right) Four normalized optical spectra of J033229.9-274530 taken on different observation dates, which always remains as optically type I. Adapted from Yang et al. (2016).

\section{Interactions between AGNs and their host galax- ies}

\subsection{AGN X-ray luminosity versus galaxy SFR}

The two relatively independent major astrophysical research fields, AGNs and galaxies, have begun to be intimately connected with each other since a series of important observational discoveries of tight relationships between $M_{\mathrm{BH}}$ and galaxy bulge properties (e.g., stellar velocity dispersion, luminosity, and mass; e.g., Magorrian et al. 1998; Ferrarese \& Merritt 2000; Gebhardt et al. 2000; Tremaine et al. 2002; Marconi \& Hunt 2003; Ferrarese \& Ford 2005; Gültekin et al. 2009; Kormendy \& Ho 2013). Subsequent results show that the volume density of SMBH accretion rate (scaled up by a factor of several thousand) closely tracks the cosmic SFR density out to at least $z \approx 2$ (e.g., Heckman et al. 2004; Merloni et al. 2004; Silverman et al. 2008a; Zheng etal. 2009; Aird et al. 2010; Mullaney et al. 2012b; Kormendy \& Ho 2013; Aird et al. 2015b), in support of the scenario of a closely related overall SMBH-galaxy growth. As a result, the co-evolution studies of galaxies and their central SMBHs have ever since aroused great interest and become a central research theme in modern astrophysics (see, e.g., Cattaneo et al. 2009; Kormendy \& Ho 2013 for a review).

The AGN X-ray luminosity is related to SMBH accretion and the galaxy SFR is related to star-formation process; as such, $L_{\mathrm{X}}$ and SFR are often taken as the observational indicators of respective growth of SMBHs and galaxies, with the relation between them providing hints for interactions between AGNs and their hosts, BH accretion modes, and likely effects of AGN feedback (see, e.g., Fabian 2012 for a review). Many studies have explored the relations between $L_{X}$ and SFR, providing a wide range of results, e.g.: (1) For $z \gtrsim 1$ moderateluminosity $\left(L_{\mathrm{X}} \approx 10^{42-44} \mathrm{erg} \mathrm{s}^{-1}\right)$ AGNs, there is generally no strong evidence for any $L_{X}$ dependence of SFR, with SFR being broadly constant over this $L_{\mathrm{X}}$ range and AGN host specific SFR (sSFR) usually being roughly consistent with main-sequence galaxies (e.g., Lutz et al. 2010; Shao et al. 2010; Mullaney et al. 2012a; Rosario etal. 2012, 2013a; Azadi etal. 2015; Stanley etal. 2015; see Fig. 13, but see Barger et al. 2015); while at $z \lesssim 1$, there appears to be a positive correlation between SFR and $L_{X}$ with such a trend being most prominent in the local universe (e.g., Shao et al. 2010; Rosario et al. 2012; Azadi et al. 2015; Lanzuisi et al. 2017; also see, e.g., Rowan-Robinson 1995; Netzer et al. 2007; Netzer 2009; Diamond-Stanic \& Rieke 2012; Matsuoka \& Woo 2015 for similar positive SFR-AGN luminosity trends; but see, e.g., Shimizu et al. 2017). (2) For $z \gtrsim 1$ highluminosity $\left(L_{\mathrm{X}} \gtrsim 10^{44} \mathrm{erg} \mathrm{s}^{-1}\right)$ AGNs, some studies suggest an increase of SFR or a roughly constant SFR toward large $L_{\mathrm{X}}$ (e.g., Hatziminaoglou et al. 2010; Lutz 
et al. 2010; Harrison et al. 2012; Rovilos et al. 2012; Santini et al. 2012; Stanley et al. 2015; Lanzuisi et al. 2017; also see, e.g., Lutz etal. 2008; Bonfield et al. 2011; Dong \& Wu 2016; Harris et al. 2016 for similar positive SFR-AGN luminosity trends), while some suggest a decrease of SFR with increasing $L_{X}$, possibly due to AGN feedback (e.g., Page et al. 2012; Barger et al. 2015).

There are several possible causes that can account, at least partially, for some of the variations between the above different observational results about moderateluminosity and high-luminosity AGNs. For example, it is always challenging to obtain robust SFR measurements, especially for high-luminosity AGNs that can produce significant FIR emission and thus contaminate that of host galaxies; limited sample sizes (i.e., poor source statistics) appear to be an unavoidable issue most of the time; and, sometimes, cosmic variance may come into play. Additionally, some physical considerations may be the more fundamental causes, e.g.: (1) The relative timescales of several processes can largely determine whether the observational signatures of suppressed star formation (due to, e.g., AGN feedback) can be maintained and thus clearly revealed; such processes include, e.g., star-formation activity, luminous AGN activity (and its variability), the delay between the onsets of star formation and AGN activity, and quenching of star formation (e.g., Di Matteo et al. 2008; Hopkins \& Quataert 2010; Gabor \& Bournaud 2013; Hickox et al. 2014; Neistein \& Netzer 2014). (2) There are likely two modes in the concomitant AGN activity and star formation that can transit from each other when conditions allow, with one mode primarily associated with low- and moderate-luminosity AGNs where the evolution is secular, with no close coupling between instantaneous AGN luminosity and current galaxy-integrated SFR, and another mode mainly associated with high-luminosity AGNs where the evolution is intense and rapid likely through (major) mergers, with tight coupling between AGN growth and host star formation (e.g., Lutz et al. 2010; Shao et al. 2010; Santini et al. 2012). (3) The exact relation between $L_{X}$ and SFR likely depends on whether the star formation is dominated by a nuclear (i.e., sub-pc scales) or an extended $(\approx \mathrm{kpc})$ component, with the former case resulting in tighter coupling than the latter (e.g., Hopkins \& Quataert 2010; Diamond-Stanic \& Rieke 2012).

Alternatively, the connection between AGN activity and star formation can be examined using a different approach from the above studies, which finds tight correlations between SFR and AGN luminosity by averaging AGN and star-formation activities over all galax- ies (irrespective of hosting an AGN or not) in a cosmological volume (e.g., Rafferty et al. 2011; Symeonidis etal. 2011; Mullaney et al. 2012b; Chen et al. 2013; Delvecchio etal. 2015). Such an approach focuses on investigating long-term average/overall behaviors, thus being immune to many detailed factors mentioned above. As a most recent example, Yang et al. (2017) study the dependence of sample-mean BH accretion rate $(<\mathrm{BHAR}>)$ on host SFR and $M_{\star}$ with a sample of $\approx 18000$ galaxies at $0.5 \leq z<2.0$, using the $7 \mathrm{Ms}$ CDF-S and CANDELS/GOODS-S observations. They compare $<$ BHAR $>$ for samples with different SFR and/or $M_{\star}$, down to levels as low as SFR $\approx 0.1 M_{\odot} \mathrm{yr}^{-1}$ and $M_{\star} \approx 10^{8} M_{\odot}$ with reasonable completeness for the first time, finding that (see Fig. 14): (1) $<$ BHAR $>$ is positively correlated with both SFR and $M_{\star}$, and both the $<$ BHAR $>$-SFR and $<$ BHAR $>-M_{\star}$ relations can be adequately fit using linear models with slope $=1$. (2) The $<\mathrm{BHAR}>-M_{\star}$ relation is tighter than the $<$ BHAR $>-$ SFR relation (confirmed by partial-correlation analyses), indicating that $\mathrm{BH}$ growth is mainly linked to $M_{\star}$ rather than SFR, and the $<\mathrm{BHAR}>-\mathrm{SFR}$ relation is largely secondary and results from the well-known star-formation main sequence (e.g., Elbaz et al. 2011). (3) Massive galaxies (i.e., $M_{\star} \gtrsim 10^{10} M_{\odot}$ ) have larger $<$ BHAR $>/<$ SFR $>$ ratios than less-massive galaxies, suggesting that the former have higher AGN fractions and/or higher SMBH fueling efficiencies than the latter (also see, e.g., Rodighiero et al. 2015). The Yang etal. (2017) results are in accordance to the SMBHgalaxy co-evolution scenario, have important implications for the $M_{\mathrm{BH}}-M_{\star}$ scaling (see Section 3.4), and make one worry about likely mass effects in at least some of the aforementioned $L_{X}-S F R$ studies that are based on direct detections.

The current research status of the relation between AGN activity and star formation is somewhat perplexing, as both the observational and theoretical pieces don't all fit together (see, e.g., Harrison 2017 for a review). Future observational studies can be improved in many aspects, e.g., more reliable SFR measurements for individual sources, broader parameter spaces (e.g., SFR, $\left.M_{\star}, L_{\mathrm{X}}, z\right)$ to be probed, better source statistics, and higher sample completeness. These improved studies will provide more sensitive tests of how AGN activity impacts star formation and make a step forward in understanding AGN triggering mechanisms.

\subsection{Conducive host galaxy properties for AGN activity}

What kinds of host galaxy environments are most AGN-friendly? This is a question that invites numerous observational efforts, which are made over a broad 

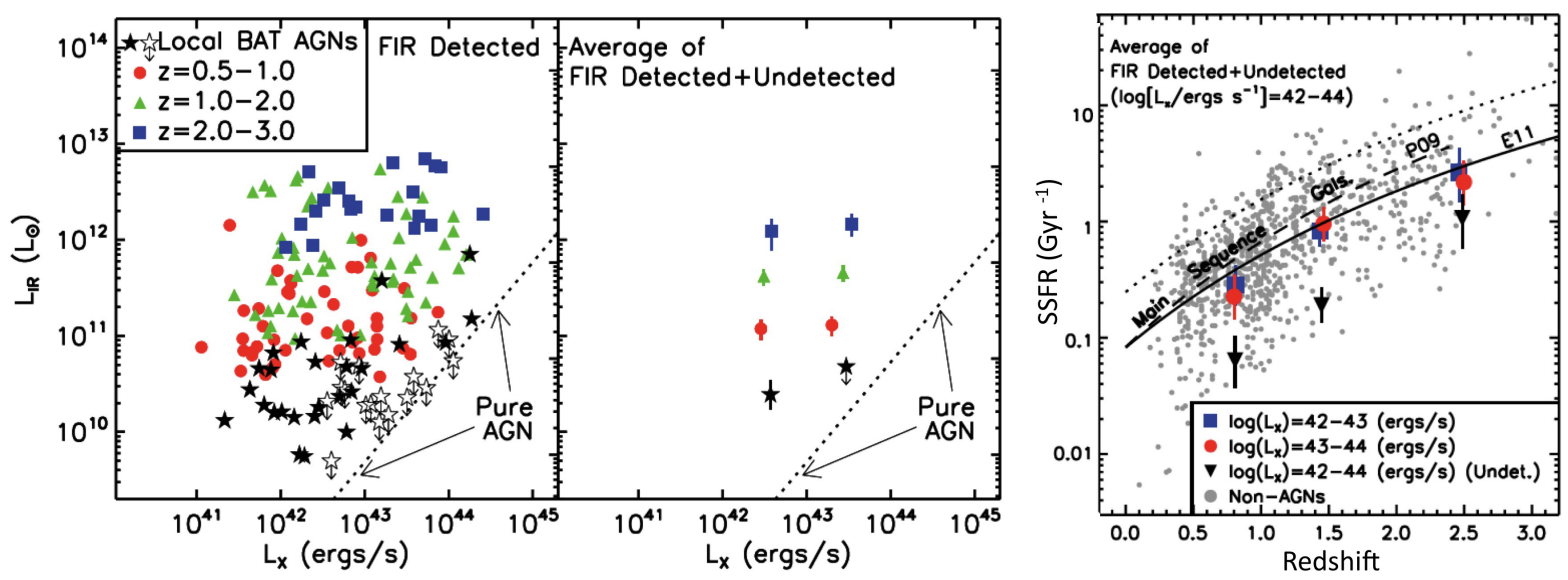

Figure 13: (Left) Total IR luminosities $\left(L_{\mathrm{IR}}\right)$ versus $L_{2-10 \mathrm{keV}}$ for the FIR detected moderate-X-ray-luminosity AGNs with $0.5<z<3.0$. Filled and open stars indicate local $(z<0.1)$ BAT/IRAS AGNs for comparison whose $60 \mu \mathrm{m}$ flux is likely dominated by the AGN rather than the host galaxy. The dotted line indicates the $L_{\mathrm{IR}}$ expected from a "pure" AGN according to Mullaney et al. (2011). (Middle) Same as left, but for the average $L_{\mathrm{IR}}$ derived from stacking analyses versus average $L_{2-10 \mathrm{keV}}$ for each $L_{\mathrm{X}}-z$ bin. Both the left and middle panels indicate no apparent $L_{\mathrm{X}}$ dependence of SFR for $0.5<z<3.0$ moderate-X-ray-luminosity AGNs. (Right) Average specific SFRs (sSFRs) of the X-ray AGN hosts versus redshift (red and blue symbols), being roughly consistent with the star-forming galaxy main-sequence trend. For comparison, the black and gray symbols indicate the stacks of the FIR-undetected AGNs and non-AGNs, respectively. The solid and dashed curves indicate the main-sequence tracks from Elbaz et al. (2011) and Pannella et al. (2009), respectively; the dotted line is a factor of 3 above the Elbaz et al. (2011) track (indicating strongly starbursting activities). Adapted from Mullaney et al. (2012a).
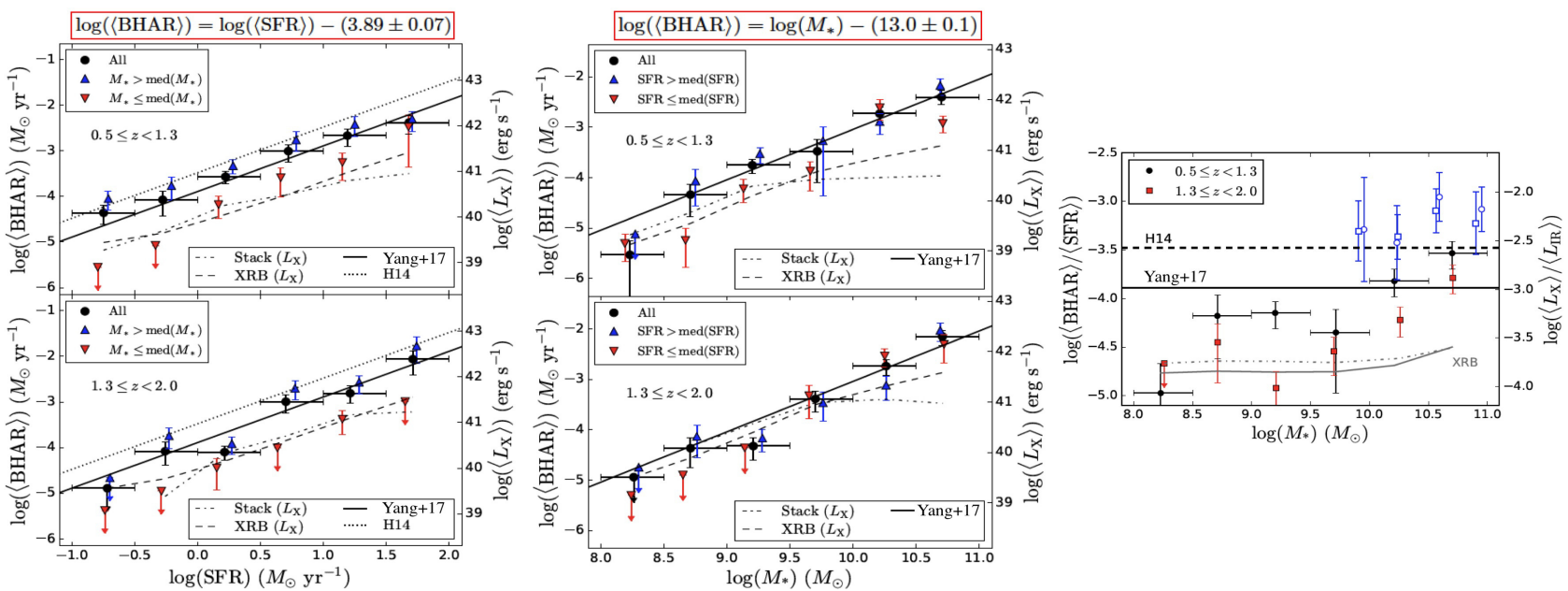

Figure 14: (Left) Average black-hole accretion rate $\left(<\mathrm{BHAR}>\right.$; converted from $<L_{\mathrm{X}}>$ that is the sum of both X-ray detected and undetected sources with galaxy contamination $L_{\mathrm{X}, \mathrm{XRB}}$ subtracted) as a function of (median) SFR for a CANDELS/GOODS-S sample in various redshift and stellar-mass bins. The solid line indicates the best-fit linear model with a fixed slope of 1 , with the equation annotated atop; the dotted line indicates the Hickox et al. (2014) model; the dashed curves indicate $L_{X, X R B}$ subtracted from each subsample; and the dash-dotted curves indicate $<L_{X}>$ for stacked sources ( $L_{\mathrm{X}, \mathrm{XRB}}$ not subtracted). (Middle) Similar to left, but for $<\mathrm{BHAR}>$ versus $M_{\star}$ in various redshift and SFR bins. The comparison between the left and middle panels indicates that the $<$ BHAR $>-M_{\star}$ relation is tighter than the $<$ BHAR $>$-SFR relation. (Right) $<$ BHAR $>\mid<$ SFR $>$ as a function of $M_{\star}$. The blue open symbols indicate the Mullaney et al. (2012b) results (circles and squares: $z \approx 1$ and $z \approx 2$ samples). The gray solid and dash-dotted curves indicate $<L_{\mathrm{X}, \mathrm{XRB}}>\mid<L_{\mathrm{IR}}>$ in the low- and high-redshift ranges, respectively. It reveals that massive galaxies have larger $<$ BHAR $>\mid<$ SFR $>$ ratios than less-massive galaxies. Adapted from Yang et al. (2017).

redshift range (up to $z \approx 3-4$ ) and obtain many interesting results, e.g.: (1) Distant X-ray AGN host galaxies are typically more massive than non-AGN galaxies, with the AGN fraction (above a given AGN cutoff lumi- nosity) increasing strongly toward larger stellar masses (e.g., Akiyama 2005; Papovich etal. 2006; AlonsoHerrero et al. 2008; Brusa et al. 2009b; Xue et al. 2010; Aird etal. 2012; Mullaney etal. 2012a; Wang et al. 
2017; see Row (d) of Fig. 15]. (2) There is a color bimodality among galaxy populations, with the majority being red(-sequence) and blue(-cloud) galaxies and the minority green(-valley) galaxies lying in between (e.g., Strateva et al. 2001; Bell et al. 2004; Brammer et al. 2009; Xue et al. 2010; see Row (a) of Fig. 15. $\mathrm{X}$-ray AGN host galaxies are generally found to be optically more luminous and redder than non-AGN galaxies (e.g., Barger et al. 2003b; Nandra et al. 2007; Silverman et al. 2008b; Xue et al. 2010; Bongiorno et al. 2012). However, when stellar mass-matched samples are considered, both AGN hosts and non-AGN galaxies are comparably luminous with similar colors, and the AGN fraction is largely constant or even slightly decreasing toward red host colors (e.g., Silverman et al. 2009; Xue et al. 2010; Rosario et al. 2013a; HernánCaballero et al. 2014; see Row (b) of Fig. 15). (3) Most $\mathrm{X}$-ray AGNs reside in star-forming and starburst hosts, with the AGN fraction increasing significantly toward higher SFRs (e.g., Silverman et al. 2009; Xue et al. 2010; Rafferty et al. 2011; Rosario et al. 2013a; Wang et al. 2017). Nevertheless, when mass-matched samples are examined, once again, the above trend of rising AGN fraction upon larger SFRs becomes less prominent or even non-detectable, and there is no apparent difference in terms of star-formation properties between AGN hosts and star-forming main-sequence galaxies (e.g., Xue etal. 2010; Mullaney etal. 2012a; see Row (c) of Fig. 15). (4) The first morphological studies of X-ray AGN hosts indicate that these AGNs preferentially reside in bulge-dominated systems (e.g., Grogin et al. 2005; Pierce etal. 2007). However, the massmatched technique does the magic once more: clear differences between morphological types of AGN hosts and non-AGN galaxies mostly disappear when the AGN and galaxy samples are matched in mass, i.e., X-ray AGNs reside in a broad range of host-galaxy types that include, e.g., disk-dominated, bulge-dominated, irregular, and point-like morphology classes (or simply categorized as undisturbed and disturbed classes; e.g., Kocevski et al. 2012; Fan et al. 2014; Villforth et al. 2014; see Fig. 16.

Based on the above X-ray AGN results, it appears clear that, among a variety of host galaxy properties (e.g., stellar mass, optical luminosity and colors, SFR, and morphology) likely conducive for AGN activity, stellar mass plays the fundamental driving role in AGN triggering, while the other properties play a secondary role; this is hardly surprising, given the masslight/luminosity (e.g., Zibetti, Charlot, \& Rix 2009), mass-color (i.e., massive galaxies generally tend to be redder; e.g., Xue et al. 2010), mass-SFR (i.e., the star-
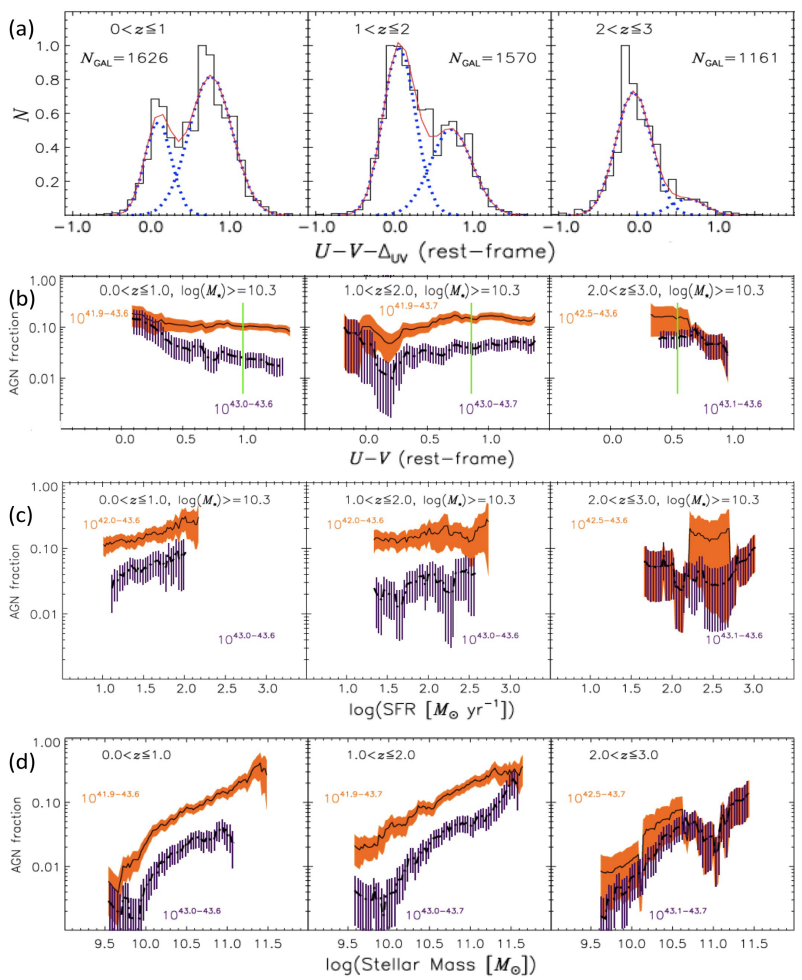

Figure 15: [Row (a)] Normalized (peak=1) histograms of rest-frame dust-extinction corrected $U-V$ color (bin size=0.1) for non-AGN galaxies (black histograms; total numbers annotated) in various redshift ranges, which all display a color bimodality. The two best-fit Gaussian components are indicated with blue dotted curves, with their sum shown as red solid curves. [Rows (b-d)] AGN fraction as a function of rest-frame $U-V$ host color (in bins of $\Delta(U-V)=0.4$ ), SFR (in bins of $\Delta \operatorname{logSFR}=0.5$ ), and $M_{\star}$ (in bins of $\Delta \log \left(M_{\star}\right)=0.5$ ) in different $z$ and $L_{X}$ bins, respectively. The green vertical lines in Row (b) roughly separate blue and red galaxies according to the Bell et al. (2004) separation scheme. The $\log \left(M_{\star}\right) \geq 10.3$ cut in Rows (b) and (c) effectively ensures mass-matched samples of AGN hosts and non-AGN galaxies for reliable comparison (see main text for details). Adapted from Xue et al. (2010).

formation main sequence; e.g., Elbaz et al. 2011), and mass-morphology (i.e., E/S0 galaxies generally dominate the higher-mass population; e.g., Bundy, Ellis, \& Conselice 2005) correlations. There are several physically plausible causes for the dominant role of stellar mass in triggering AGN activity, e.g., massive galaxies (1) have stronger gravitational pull to make gas fall into galaxy centers and fuel SMBHs eventually, (2) are more likely to have nuclear bars to induce gas inflow efficiently, and (3) tend to have larger SMBHs that are more capable of accreting gas from their vicinity (see, e.g., Section 4.2 of Yang et al. 2017 for details). Therefore, the conditions that are most conducive for AGN activity appear to be a massive host galaxy and a large reservoir 


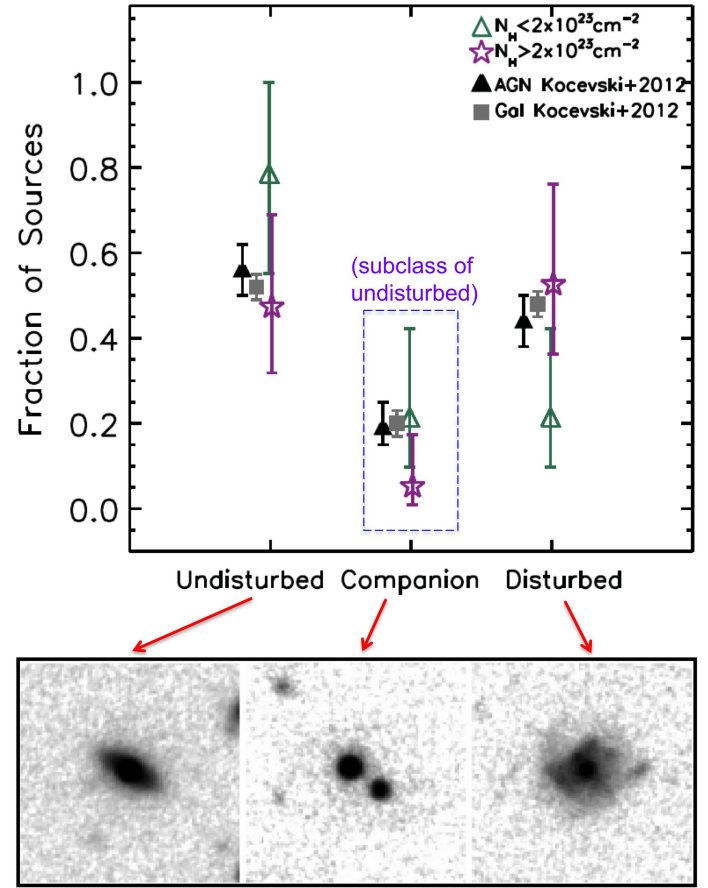

Figure 16: (Top) Fraction of $z \approx 2$ unobscured/moderately obscured (green symbols) and highly obscured (purple symbols) MIR-luminous quasar hosts with various disturbance classes that are classified using the HST F160W images (see Bottom for example 6 arcsec $\times 6$ arcsec cutouts). The Kocevski et al. (2012) $z \approx 2$ AGN (black symbols) and non-AGN (gray symbols) samples are shown for comparison. Adapted from Del Moro et al. (2016)

of gas (e.g., Silverman et al. 2009; Vito et al. 2014b). In combination with the fact that galaxy stellar masses can be estimated more reliably than colors and luminosities (e.g., van Dokkum et al. 2006), consequently, evolutionary studies of galaxies and SMBHs are best probed using stellar-mass selected samples (e.g., van Dokkum et al. 2006; Kriek et al. 2008) and/or mass-matched samples (e.g., Silverman et al. 2009; Xue et al. 2010). As such, the digesting of the above results derived with mass-matched samples plainly indicates at least two points: (1) there is essentially no substantial difference between many physical properties (e.g., optical luminosity and colors, SFR, and morphology) of X-ray AGN hosts and coeval non-AGN galaxies over a broad range of redshift (but see, e.g., Mullaney et al. 2015b; Wang et al. 2017); and (2) currently there appears no strong direct observational evidence for feedback effects from moderate-luminosity X-ray AGNs (e.g., Xue et al. 2010; Bongiorno et al. 2012; Rosario et al. 2013b; Azadi et al. 2017; but see, e.g., Wang et al. 2017) 10

\footnotetext{
${ }^{10}$ Interestingly, theoretical studies predict diverse roles played by
}

Gas-rich major mergers and triggering of distant AGNs are often closely related in theoretical considerations (e.g., Sanders et al. 1988; Di Matteo et al. 2005; Hopkins et al. 2008). Observationally, however, only $\lesssim 20 \%$ of the AGN population at $z \approx 0-2.5$ have clear signatures of major mergers (e.g., Koss et al. 2010; Silverman et al. 2011; Kocevski et al. 2012; Cotini et al. 2013; Villforth et al. 2014), which is much below theoretical expectations. Interestingly, recent morphological studies find that major mergers only trigger the most luminous AGNs (e.g., Treister et al. 2012; Rumbaugh et al. 2017; but see, e.g., Villforth et al. 2017); and highly obscured AGN hosts tend to undergo dynamical compaction (e.g., Chang et al. 2017), interactions, or mergers (e.g., Kocevski et al. 2015; Lanzuisi et al. 2015a; Del Moro et al. 2016). In particular, Del Moro et al. (2016) study a sample of $z \approx 2$ MIR-luminous quasars, and find that the highly obscured quasars tend to reside in galaxies with disturbed morphologies while the unobscured/moderately obscured quasars preferentially lie in undisturbed hosts, but the disturbed quasar hosts only constitute the minority $(\approx 40 \%)$ of the entire sample (see Fig. 16), which is consistent with other findings (e.g., Kocevski et al. 2015; Lanzuisi et al. 2015a). The above results indicate that secular processes such as galaxy bars and disk instabilities, as well as minor mergers, may be the main fueling mechanisms for majority AGN populations. Therefore, it remains appealing for future morphological studies to quantify reliably and accurately the respective contribution of each likely AGN fueling mechanism as a function of, e.g., $L_{X}$ and redshift.

\subsection{SMBH growth behavior revealed by $\Gamma-\lambda_{\mathrm{Edd}}$ rela- tion and $\lambda_{\text {Edd }}$ distribution}

$\lambda_{\text {Edd }}$ is an important parameter in accretion disk theories, which can be used as a primary indicator of SMBH growth rate and accretion mode ${ }^{11}$ Recent observational results about the AGN $\Gamma-\lambda_{\text {Edd }}$ relation reveal a Vshape correlation: as $\lambda_{\text {Edd }}$ decreases continuously, the $\Gamma-\lambda_{\text {Edd }}$ correlation changes from being positive (e.g., Wang et al. 2004; Shemmer et al. 2006, 2008; Risaliti etal. 2009; Zhou \& Zhao 2010; Brightman et al. 2013; Fanali et al. 2013; Yang et al. 2015) into being

moderate-luminosity AGNs, whose feedback may be either important (e.g., Fabian et al. 2008; Raimundo et al. 2010) or not (e.g., Hopkins $\&$ Hernquist 2006).

${ }^{11}$ In practice, the observable specific accretion rate (i.e., $L_{\mathrm{X}} / M_{\star}$ ) is often taken as a proxy for $\lambda_{\text {Edd }}$; thus, for convenience and succinctness, the mixed use of $L_{\mathrm{X}} / M_{\star}$ and $\lambda_{\text {Edd }}$ is adopted here, i.e., $\lambda_{\mathrm{Edd}}$ actually refers to $\lambda_{\text {Edd }}$ itself or $L_{X} / M_{\star}$. 
negative (e.g., Constantin et al. 2009; Gu \& Cao 2009; Younes et al. 2011; Jang et al. 2014; Yang et al. 2015; Kawamuro et al. 2016), with the transition occurring at $\lambda_{\text {Edd }} \approx 1 \% \sqrt{12}$ It is generally believed that these observational results reflect that, as the accretion rate decreases, the SMBH accretion mode changes accordingly, from the original standard thin accretion disk into radiatively inefficient accretion flow (e.g., Gu \& Cao 2009; Brightman etal. 2013; Jang etal. 2014; Yuan \& Narayan 2014). Recently, Yang et al. (2015) propose a coupled hot accretion flow-jet model, which can well explain the overall $\Gamma-\lambda_{\text {Edd }}$ correlation and even predicts that, toward the very low $\lambda_{\text {Edd }}$ regime (e.g., $\lambda_{\text {Edd }} \lesssim 10^{-5}$ ), this correlation will display a small bump and then level off eventually. Interestingly, Liu et al. (2016b) find that, toward the very high $\lambda_{\text {Edd }}$ regime (e.g., $\lambda_{\text {Edd }} \gtrsim 0.1$ ), the $\Gamma-\lambda_{\text {Edd }}$ correlation appears to be largely constant or even negative, which is different from previous findings. Therefore, it would be useful to build a large, uniform, and (relatively) complete AGN sample with, e.g., the CDF observations in conjunction with other data, to study in detail the $\Gamma$ - $\lambda_{\text {Edd }}$ correlation and its likely dependence on redshift and host galaxy properties (Sun et al. in prep.), in order to thoroughly compare observations with various SMBH accretion models. If reliably calibrated (see, e.g., Section 4.3 of Brandt \& Alexander 2015 for the challenges in obtaining reliable estimates of $\Gamma$ and $\lambda_{\text {Edd }}$ ), the $\Gamma-\lambda_{\text {Edd }}$ correlation may be used to conveniently estimate some important AGN parameters (e.g., $\lambda_{\mathrm{Edd}}, M_{\mathrm{BH}}$, and mass accretion rate), which will facilitate better understanding accretion processes and evolution of AGNs.

Observational $\lambda_{\text {Edd }}$ estimates for $z \approx 0-4$ X-ray AGNs span a broad range of $\lambda_{\mathrm{Edd}} \approx 10^{-5}-1$, with the majority having $10^{-4}-0.1$ (e.g., Babić et al. 2007; Ballo et al. 2007; Alonso-Herrero et al. 2008; Brusa et al. 2009b; Hickox et al. 2009; Raimundo et al. 2010; Trump et al. 2011; Lusso et al. 2012; Matsuoka et al. 2013; Azadi et al. 2015; Suh et al. 2015; Bernhard et al. 2016). On average, $\lambda_{\text {Edd }}$ seems to increase with redshift at any given $M_{\mathrm{BH}}$, but has no clear evolution with redshift at any given $L_{\text {bol }}$ (e.g., Lusso et al. 2012). Further careful analyses reveal that the intrinsic $\lambda_{\text {Edd }}$ distribution for X-ray AGNs appears to follow a "universal" powerlaw with a slope independent of both $M_{\star}$ and redshift out to $z \approx 2.5$ (Aird et al. 2012, 2013; Bongiorno et al.

\footnotetext{
${ }^{12}$ See, e.g., Fig. 1 of Gu \& Cao (2009) and Fig. 2 of Yang et al. (2015) for a demonstration of the V-shape $\Gamma$ - $\lambda_{\text {Edd }}$ correlation, which is also seen in some well-observed individual AGNs (e.g., Sobolewska \& Papadakis 2009; Emmanoulopoulos et al. 2012; Connolly et al. 2016) as well as in XRB populations (e.g., Yang et al. 2015).
}

2012; Azadi et al. 2015; Wang et al. 2017), implying that the basic physical processes responsible for triggering and fueling the overall AGN population might be essentially the same; such a power-law distribution is in contrast to a lognormal distribution, with the former also seen for, e.g., AGNs in quiescent galaxies (e.g., Kauffmann \& Heckman 2009; but see, e.g., Jones et al. 2016) and the latter seen for, e.g., the observed $\lambda_{\text {Edd }}$ distribution for X-ray AGNs (e.g., Aird et al. 2012) as well as the $\lambda_{\text {Edd }}$ distribution for optically luminous AGNs (e.g., Kollmeier et al. 2006) and AGNs in star-forming galaxies (e.g., Kauffmann \& Heckman 2009).

Specifically, Wang et al. (2017) study the $\lambda_{\text {Edd }}$ distribution $\left(\mathrm{p}\left(\lambda_{\text {Edd }}\right)\right)$ for a mass-complete sample of $0.5<$ $z<2.5$ moderate-luminosity X-ray AGNs in the two GOODS fields, and find some interesting results (see Fig. 17): (1) $\mathrm{p}\left(\lambda_{\text {Edd }}\right)$ for the overall galaxy population appears to be a power-law with a slope of -0.4 , being consistent with the slope obtained by Jones et al. (2016) but slightly shallower than the slope of -0.6 measured by, e.g., Aird et al. (2012). (2) However, in terms of redshift evolution, $\mathrm{p}\left(\lambda_{\mathrm{Edd}}\right)$ is different for galaxies with different intrinsic colors, such that red galaxies having more rapid redshift evolution (i.e., $\mathrm{p}\left(\lambda_{\mathrm{Edd}}\right) \propto$ $(1+z)^{3.7}$, agreeing with previous results for the overall galaxy population; e.g., Aird et al. 2012; Bongiorno et al. 2012) than both blue and green galaxies (note that $\mathrm{p}\left(\lambda_{\mathrm{Edd}}\right) \propto(1+z)^{1.8}$ for the overall galaxy population, which includes red, green, and blue galaxies). (3) There is marginal evidence for red galaxies having a steeper power-law slope $(-0.6)$ of $\mathrm{p}\left(\lambda_{\text {Edd }}\right)$ than both blue and green galaxies, with the former slope in agreement with previous measurements (e.g., Kauffmann \& Heckman 2009; Aird etal. 2012). The Wang et al. (2017) results reveal the strong dependence of SMBH accretion on their host colors, and thus caution that ambiguous conclusions may be drawn without taking such a color dependence into account.

All the above works on X-ray AGN $\lambda_{\text {Edd }}$ distribution suffer, to various degrees, from issues such as limited sample sizes, in particular toward higher redshifts and lower X-ray luminosities, thus resulting in conclusions associated with large uncertainties. Therefore, future studies using larger and relatively complete samples, more uniform AGN identification criteria, and more reliable $\lambda_{\text {Edd }}$ estimates would be critical to nail down the detailed behavior of intrinsic X-ray AGN $\lambda_{\text {Edd }}$ distribution as a function of, e.g., redshift, host stellar mass, and intrinsic colors, thereby providing sharp insights into SMBH growth processes across cosmic time. 

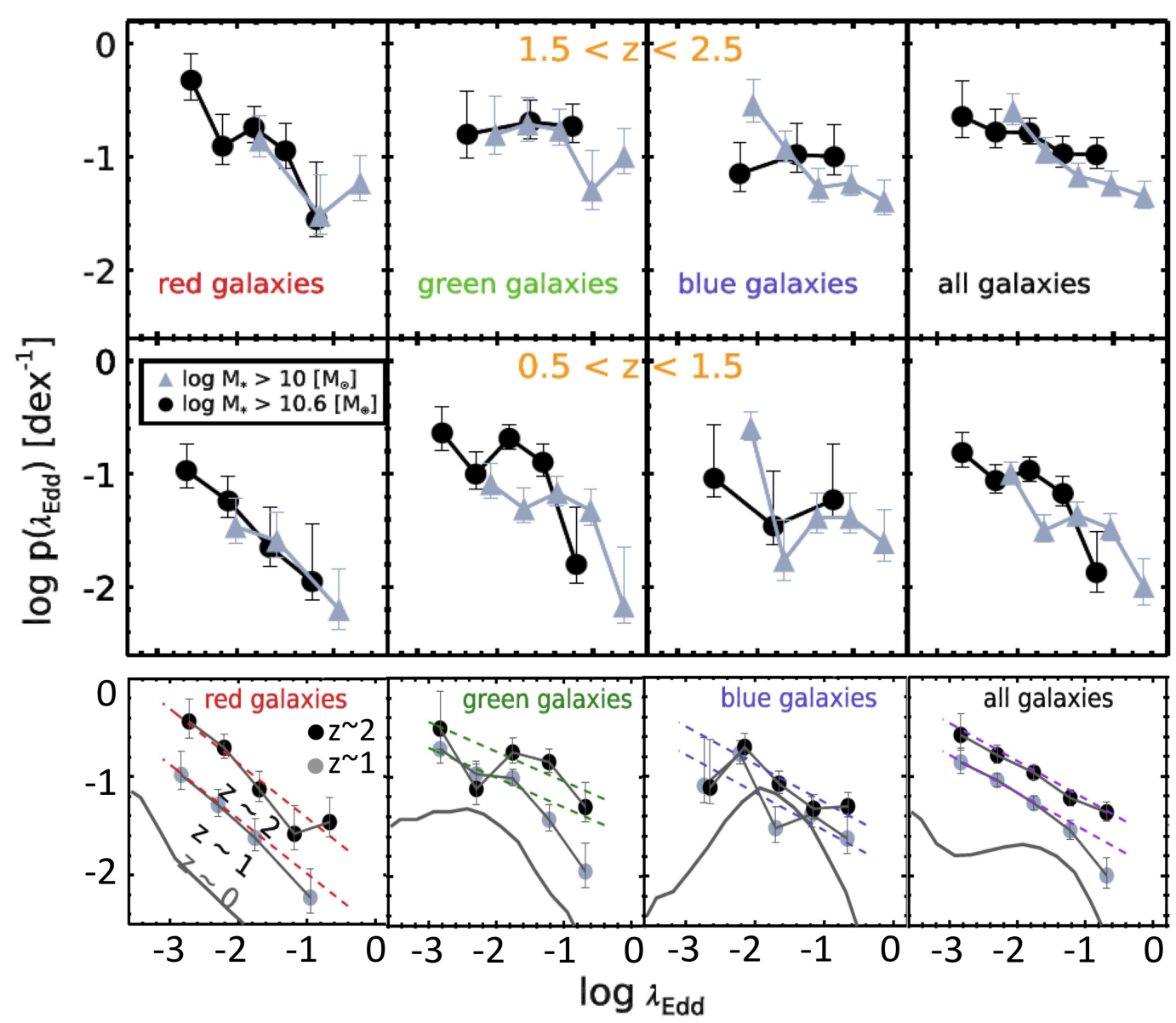

Figure 17: Binned Eddington ratio distributions ( $\mathrm{p}\left(\lambda_{\mathrm{Edd}}\right)$, i.e., the probability of galaxies hosting an AGN with $\lambda_{\text {Edd }}$ in a given $M_{\star}$ and $z$ bin, with $1 / V_{\max }$ correction applied) for a sample of moderate-luminosity $\left(L_{0.5-8 \mathrm{keV}}=10^{41.9-43.7} \mathrm{erg} \mathrm{s}^{-1}\right)$ AGNs in various bins of redshifts, $M_{\star}$, and intrinsic (i.e., dust-extinction corrected) host colors. In the bottom panel, no $M_{\star}$ subdivision is applied; the best-fit $\mathrm{p}\left(\lambda_{\mathrm{Edd}}\right)$ models at two redshift ranges $(z \approx 1$ and $z \approx 2)$ are shown as dashed lines based on the Extended Maximum Likelihood fitting of the unbinned data; and the gray curves indicate the observed binned $\mathrm{p}\left(\lambda_{\text {Edd }}\right)$ of local Type II AGN hosts from Kauffmann \& Heckman (2009). It seems clear that there is a strong dependence of SMBH accretion ( $\mathrm{p}\left(\lambda_{\mathrm{Edd}}\right)$ as a proxy here) on the host colors (see main text for details). Adapted from Wang et al. (2017).

\subsection{Coeval growth of SMBHs and their hosts}

The coeval growth of SMBHs and their host galaxies is vividly reflected (in part) in the observed tight $M_{\mathrm{BH}}-M_{\text {bulge }}$ correlation (e.g., Kormendy \& Gebhardt 2001; McLure \& Dunlop 2002; Marconi \& Hunt 2003; Ferrarese \& Ford 2005; Lauer et al. 2007; Gültekin et al. 2009; Sani et al. 2011; Vika et al. 2012), which is largely in the form of $M_{\mathrm{BH}} \sim M_{\text {bulge }}^{1.2}$ (e.g., Wandel 1999; Häring \& Rix 2004; Kormendy \& Ho 2013; McConnell \& Ma 2013) with an intrinsic scatter of $\approx 0.3$ dex (e.g., Kormendy \& Ho 2013). The tight $M_{\mathrm{BH}}-M_{\text {bulge }}$ correlation can be explained either by AGN feedback where the coupling between AGN triggering and feedback-regulated star formation plays a key role (e.g., Di Matteo et al. 2005; Hopkins et al. 2006b; Si- jacki et al. 2007; Booth \& Schaye 2009; Fabian 2012), or by galaxy mergers based on the central limit theorem (e.g., Peng 2007; Hirschmann et al. 2010; Jahnke $\&$ Macciò 2011). In addition to the $M_{\mathrm{BH}}-M_{\text {bulge }}$ relation, many works study the $M_{\mathrm{BH}}-M_{\star}$ relation instead and find similar tight correlations (e.g., Kormendy \& Richstone 1995; Magorrian et al. 1998; Gültekin et al. 2009; Merloni et al. 2010; Schulze \& Gebhardt 2011; McConnell \& Ma 2013; Reines \& Volonteri 2015; Sun et al. 2015), given the facts that $M_{\star}$ is an important physical property (see Section 3.2) being relatively easy to measure, $M_{\text {bulge }}$ is approximately equal to $M_{\star}$ for bulge-dominated systems, and it is challenging to separating $M_{\text {bulge }}$ from $M_{\star}$ at $z \gtrsim 1$. Together, scaling relations between SMBHs and their hosts (including, 
e.g., $M_{\mathrm{BH}}-M_{\text {bulge }}$ and $\left.M_{\mathrm{BH}}-M_{\star}\right)$ are fundamentally important to understanding $\mathrm{SMBH}$ and galaxy evolution across cosmic time.

Obviously, observational studies of the evolution of the $M_{\mathrm{BH}}-M_{\star}\left(M_{\text {bulge }}\right)$ relation are of great interest yet with diverse results. For example, many works find that the $M_{\mathrm{BH}} / M_{\star}\left(M_{\mathrm{BH}} / M_{\text {bulge }}\right)$ ratio evolves positively with redshift, with high-redshift SMBHs being a factor of several overmassive relative to their host galaxies compared to their local counterpart systems (e.g., Peng et al. 2006a, 2006b; Ho 2007; Merloni et al. 2010; Bennert et al. 2011; Bongiorno et al. 2014; Shankar et al. 2016). However, some other works find no difference in the $M_{\mathrm{BH}} / M_{\star}$ ratio between high-redshift and local measurements (e.g., Jahnke et al. 2009; Schramm \& Silverman 2013; Sun et al. 2015). Additionally, there are works finding high-redshift undermassive SMBHs that are located below the local scaling relation (e.g., Borys et al. 2005; Alexander et al. 2008b; Urrutia et al. 2012). The likely causes for the above inconsistent results are manifold, e.g., poor statistics due to limited sample sizes, use of unrepresentative subsamples, lack of corrections for observational biases (e.g., Eddington bias, biased $M_{\mathrm{BH}}$ estimates for high-redshift sources, fluxlimited samples being biased toward larger $M_{\mathrm{BH}} / M_{\star}$ ratios; e.g., Lauer etal. 2007; Shen \& Kelly 2010; Schulze \& Wisotzki 2011, 2014; Bongiorno et al. 2014; Shankar et al. 2016), as well as large uncertainties associated with $M_{\mathrm{BH}}$ measurements (sometimes also $M_{\star}$ estimates, e.g., for luminous quasars; e.g., Bongiorno et al. 2012; Shen 2013).

Recently, Sun et al. (2015) carry out a unique study that simultaneously measures high-redshift $M_{\mathrm{BH}}-M_{\star}$ relation and its instantaneous evolution (i.e., SMBH and galaxy growth rates), using a sample of 69 Herscheldetected broad-line AGNs at $0.2 \leq z<2.1$ from the COSMOS and CDF-S fields and accounting for biases due to both sample selection limits and the steep slope of $M_{\star}$ distribution at the high-mass end (see Fig. 18). They find: (1) There is no evolution in the $M_{\mathrm{BH}}-M_{\star}$ relation from $z \approx 2$ to the present time. (2) The $M_{\mathrm{BH}}-M_{\star}$ relation has a "self-maintenance" function, i.e., AGNs and their hosts lying off the $M_{\mathrm{BH}}-M_{\star}$ relation (i.e., "outliers") tend to have evolutionary vectors anti-correlated with their $M_{\mathrm{BH}} / M_{\star}$ ratios, such that they will evolve back toward the canonical $M_{\mathrm{BH}}-M_{\star}$ track given sufficient amounts of evolutionary time. (3) The preferred AGN duty cycle that best maintains the non-evolving $M_{\mathrm{BH}}-M_{\star}$ relation appears to be a non-evolving (i.e., within a factor of $\approx 4$ ) value of $\approx 0.1$. These results are in line with the scenario that the same gas reservoir fuels both SMBH accretion and galaxy star formation,
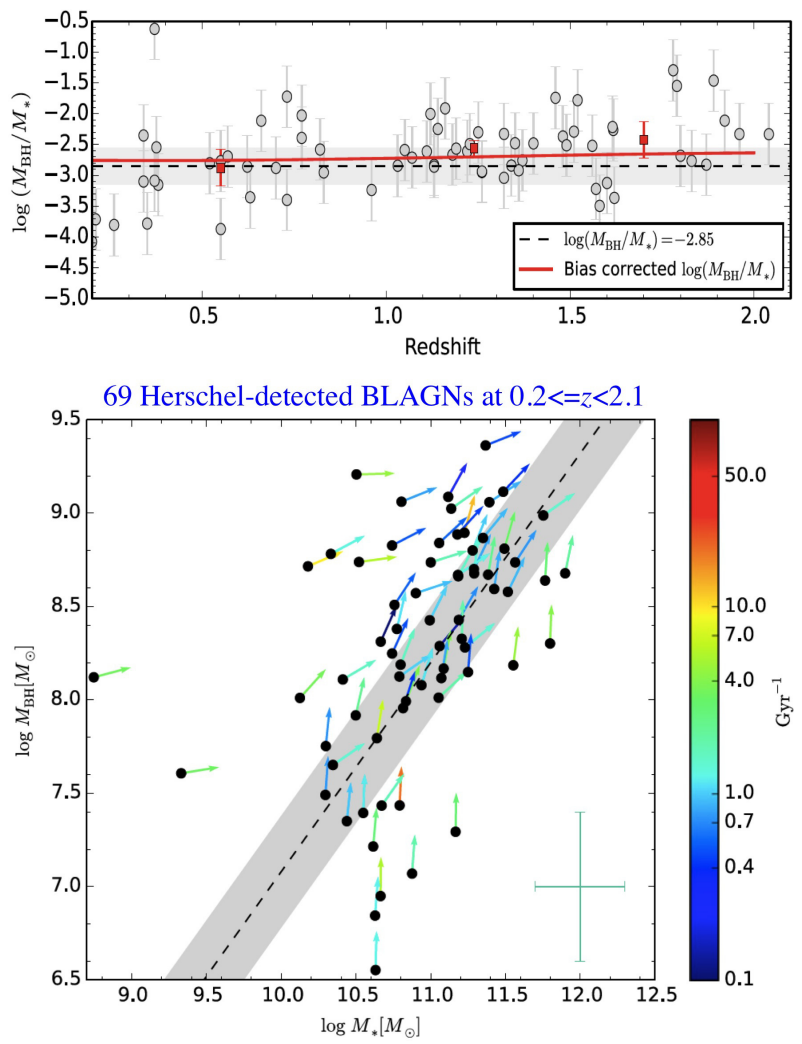

Figure 18: (Top) $M_{\mathrm{BH}} / M_{\star}$ as a function of $z$. The three red squares indicate average $M_{\mathrm{BH}} / M_{\star}$ for sources with $z<1.0,1.0 \leq z<1.5$, and $z \geq 1.5$. The red solid line indicates the local Häring \& Rix (2004) $M_{\mathrm{BH}}-M_{\text {bulge }}$ relation $\left(M_{\text {bulge }} \approx M_{\star}\right.$ here as these local host galaxies are bulge-dominated; dashed line; the shaded region indicates its uncertainty) as it would be observed under the biases of the sample presented here. No evolution of the $M_{\mathrm{BH}}-M_{\star}$ relation is observed at $0.2 \leq z<2.1$. (Bottom) "Flow patterns" of SMBHs and their host galaxies in the $M_{\mathrm{BH}}-M_{\star}$ plane, with arrows showing the evolutionary directions and colors indicating the absolute value of the total specific growth rate (i.e., $\sqrt{\mathrm{s} \dot{M}^{2}+\mathrm{sSFR}^{2}}$, where $\mathrm{s} \dot{M}$ is the specific $\mathrm{BH}$ mass accretion rate). The large cross indicates $1 \sigma$ uncertainties of $M_{\mathrm{BH}}$ and $M_{\star}$. The self-maintenance behavior of the $M_{\mathrm{BH}}-M_{\star}$ relation is evident. Adapted from Sun et al. (2015).

and have important implications for mass assembly histories of SMBHs and their host galaxies.

Future studies on the $M_{\mathrm{BH}}-M_{\star}$ scaling relation can gain further insights with higher confidence levels on the formation and co-evolution history of SMBHs and their hosts, by enlarging samples of representative AGNs, controlling for various observational biases, and reducing uncertainties associated with $M_{\mathrm{BH}}$ estimates. Furthermore, additional distinct constraints upon the connection between SMBHs and their host galaxies can be placed, by improving measurements of host galaxy properties (e.g., SFR, morphology, gas content) and 
then examining likely dependence of the $M_{\mathrm{BH}}-M_{\star}$ scaling relation on them, thereby further unraveling their co-evolutionary details.

\section{Evolution of starburst and normal galaxy X-ray emission}

Remarkable insights into the formation and evolution of populations of XRBs have been gained through Chandra studies of local galaxies (see, e.g., Fabbiano 2006 for a review); only ultradeep Chandra observations have enough sensitivity to study the X-ray properties of starburst and normal galaxies at large cosmological distances either by individually detecting them or by sensitively stacking their signal, thus providing a direct view of the cosmic history of XRB production and evolution. Indeed, the CDFs have detected a significant X-ray galaxy population, e.g., $28.3 \%$ of the $10087 \mathrm{Ms}$ CDF-S main-catalogs sources are galaxies with $0.038 \leq$ $z \leq 2.636$ (see Table 1). As predicted by previous X-ray number-counts studies, the contribution of galaxies to the cumulative X-ray number counts is rapidly rising toward low fluxes, and galaxies outnumber AGNs in the SB for the first time around the $7 \mathrm{Ms}$ CDF-S flux limits (see Fig. 31 of Luo et al. 2017 and Section 2.1). These detections will enable substantial improvement of galaxy XLF characterization and detailed study of XLF dependence upon redshift, galaxy morphology, type, SFR, and stellar age (e.g., Georgakakis et al. 2006; Ptak et al. 2007; Tremmel et al. 2013; Lehmer et al. 2014). Furthermore, Chandra stacking analyses have revealed evolution of the high-mass X-ray binary (HMXB) populations in late-type galaxies as a response to the rapidly increasing cosmic SFR with redshift (e.g., Reddy et al. 2005; Laird et al. 2006; Lehmer et al. 2008; Cowie et al. 2012): $z \approx 0.5-1.4$ late-type galaxies are 5-15 times more X-ray luminous (per unit stellar mass) than their local counterparts, showing evidence for "downsizing" of XRB populations. In contrast, stacking results of luminous early-type galaxies indicate little evolution of their hot interstellar gas up to $z \approx 1.2$, providing insights into how AGN activity keeps the long-term balance between gas heating and cooling (e.g., Danielson et al. 2012).

A number of studies based on Chandra observations have constrained scaling relations between $L_{\mathrm{X}}$ and SFR for HMXB populations (i.e., $\left.L_{X}(\mathrm{HMXB}) / \mathrm{SFR}\right)$ and between $L_{\mathrm{X}}$ and $M_{\star}$ for low-mass $\mathrm{X}$-ray binary (LMXB) populations (i.e., $L_{X}(\mathrm{LMXB}) / M_{\star}$; e.g., Bauer et al. 2002a; Ranalli et al. 2003; Colbert et al. 2004; Hornschemeier et al. 2005; Lehmer et al. 2010; Boroson et al. 2011; Mineo et al. 2012, 2014; Vattakunnel et al. 2012; Zinn et al. 2012; Basu-Zych etal. 2013; Symeonidis et al. 2014). However, the large scatters in these X-ray scaling relations cannot be accounted for by measurement errors and/or statistical fluctuations (e.g., Hornschemeier et al. 2005; Mineo et al. 2012), and hence point to some real physical variations of, e.g., stellar ages, metallicities, and star formation histories that likely influence XRB formation and evolution significantly (see, e.g., Madau \& Dickinson 2014 for a review). Fragos et al. (2013a) present a much improved framework of theoretical XRB populationsynthesis models that is supported by many subsequent observational tests, being able to track XRB population evolution throughout cosmic history, make predictions for redshift evolution of the scaling relations, and identify a "best-fit" theoretical model for the local scaling relations. With the CDF and new deep multiwavelength data available, it is now plausible to use stacking techniques to isolate large populations of galaxies, obtain their global physical properties, investigate their population-averaged X-ray emission, and compare with XRB population-synthesis models in great detail (e.g., Laird et al. 2006; Lehmer et al. 2007, 2008; Cowie et al. 2012; Basu-Zych et al. 2013).

Recently, Lehmer et al. (2016) make use of the $6 \mathrm{Ms}$ CDF-S data through reliable X-ray stacking analyses to examine the dependence of galaxy XRB emission on SFR, $M_{\star}$, and redshift (in the range of $z \approx 0$ 7 ), thereby conducting the most powerful and robust tests to date of the Fragos et al. (2013) model predictions. Their findings are as follows: (1) Scaling relations involving SFR, $M_{\star}$, and redshift simultaneously best characterize global galaxy X-ray emission $\left(L_{X}\right)$, in stark contrast to a widely assumed "universal" $L_{\mathrm{X}} / \mathrm{SFR}$ relation (see Fig. 19). (2) HMXB and LMXB populations appear to evolve as $L_{2-10 \mathrm{keV}}(\mathrm{HMXB}) / \mathrm{SFR} \propto$ $(1+z)$ and $L_{2-10 \mathrm{keV}}(\mathrm{LMXB}) / M_{\star} \propto(1+z)^{2-3}$, respectively, at least up to $z \approx 2.5$, which is consistent with basic XRB population-synthesis model predictions that attribute the increase in the HMXB/LMXB relation with redshift primarily to effects related to the decrease in metallicities/stellar ages (Fragos et al. 2013a; also see Aird, Coil, \& Georgakakis 2017). However, the marginal agreement between the observational data and the Fragos et al. (2013) best-fit model necessitates minor revisions of such XRB population-synthesis models (see Fig. 19. (3) LMXBs likely dominate galaxy X-ray emissivity (i.e., $L_{X}$ per volume) out to $z \approx 1-2$, while HMXBs take over at higher redshifts. (4) The overall galaxy X-ray emissivity peaks around $z \approx 1.5-3$, mimicking the cosmic SFR density, but declines more slowly at $z \gtrsim 3$ than the latter owing to the rising $L_{X} / S F R$ 
scaling with redshift; extrapolation of these results indicates that galaxies provide a larger X-ray emissivity than AGNs at $z \gtrsim 6-8$, thereby dominating the reionization process, as expected by XRB population-synthesis models (see, e.g., Fragos et al. 2013a, 2013b; also see, e.g., Vito et al. 2016 mentioned in Section 2.2.

This Lehmer et al. (2016) work, together with many previous relevant studies, can be significantly improved, e.g., by utilizing statistically significant samples of local (through direct detections) and distant galaxies (mainly relying on stacking techniques) with a variety of physical characteristics (e.g., $M_{\star}$, SFR, stellar age, metallicity, and morphology), from an optimal combination of current and scheduled, wide and deep Chandra surveys. Without question, these works will be greatly boosted even further, thanks to the employment of future powerful X-ray observatories (see Section 7).

\section{Census of X-ray galaxy groups and clusters}

Theoretically, there is almost no doubt that the growth and evolution of SMBHs and galaxies are environmentally dependent (e.g., Kauffmann 1996; De Lucia et al. 2006). However, in terms of detailed observational manifestations, it is still not so clear about the exact role that large-scale structures (LSSs; including galaxy groups, clusters, and superclusters) play regarding AGN triggering and star-formation activities (see, e.g., McNamara \& Nulsen 2012 for a review on AGN feedback in LSSs), which is often complicated by additional likely dependencies such as host stellar mass, AGN luminosity, and redshift. Galaxy groups and clusters, lying at the high end of the cosmic density spectrum, have been intensively observed by X-ray observations that are subject to the least observational biases (e.g., Brandt et al. 2001; Bauer et al. 2002b; Giacconi et al. 2002; Finoguenov et al. 2006, 2007, 2010, 2015; Silverman et al. 2010; Allen, Evrard, \& Mantz 2011; Tanaka et al. 2012, 2013; Ehlert et al. 2013, 2014, 2015; Patel et al. 2015; Koulouridis et al. 2016; Miniati et al. 2016; Rumbaugh etal. 2017), given the utility of extragalactic X-ray surveys (see Section 1.1); but studies based on such observations sometimes obtain inconsistent results. For example, significant suppression of X-ray AGNs in clusters (e.g., lower AGN fractions than in field populations) has been observed by some authors (e.g., Koulouridis \& Plionis 2010; Haines et al. 2012; Ehlert et al. 2013, 2014), but not seen by other authors (e.g., Martini et al. 2013; Melnyk et al. 2013; Koulouridis et al. 2014). Therefore, more observational efforts are needed to better understand the interplay between AGN and star-formation activities and their sur- rounding structures, and hopefully reconcile the inconsistent results.

Compared to X-ray clusters, X-ray galaxy groups prove to be more challenging objects to study in X-rays, which essentially rely on X-ray observations with larger depths. Fortunately, both Chandra and XMM-Newton observations have elevated the research field of galaxy groups to a new level, with many surveys producing large catalogs of X-ray selected groups over a wide redshift range of $0<z<1.5$ (e.g., Brandt et al. 2001; Bauer et al. 2002b; Giacconi etal. 2002; Finoguenov et al. 2007, 2009, 2010; Bielby et al. 2010). One of the current focuses is to accumulate a statistical sample of high-redshift (i.e., $z \gtrsim 1.5$ ) groups, as they are likely progenitors of the local clusters in a statistical sense, thus being key to better understand the origin of the environmental dependence seen locally. In this regard, a number of such systems up to $z \approx 2$ have been discovered, although some of these systems need further spectroscopic observations for confirmation, e.g.: Papovich et al. (2010) and Tanaka et al. (2010) independently confirm a $z=1.62$ group in the Subaru/XMMNewton Deep Field; Henry et al. (2010) report a possible $z=1.75$ X-ray group; Gobat et al. (2011) find a $z=2.07$ group by color selection; and Andreon et al. (2009) and Spitler etal. (2012) present a few $z \approx 2$ groups based on $z_{\text {phot }}$ selection. These high-redshift groups, combined with those at lower redshifts, provide an ideal resource to understand the evolution of groups and examine galaxy formation and evolution across the environments and cosmic time, and is also a powerful probe of cosmology (e.g., Finoguenov et al. 2010, 2015).

Recently, Finoguenov et al. (2015) combine the ultradeep Chandra and XMM-Newton observations in the CDF-S/E-CDF-S to carry out a systematic search for $\mathrm{X}$-ray groups down to an unprecedented flux level of $2 \times 10^{-16}$ erg $\mathrm{cm}^{-2} \mathrm{~s}^{-1}$, with extensive spectroscopic observations providing identifications of galaxy groups out to high redshifts. They produce an X-ray group catalog that consists of a total of 46 spectroscopically identified systems up to $z=1.61$ and down to low masses (see the left panel of Fig. 20, and investigate their properties, finding that: (1) The number counts and XLF of the E-CDF-S X-ray groups are in broad agreement with expectations of the $\Lambda \mathrm{CDM}$ cosmological model (also see, e.g., Liu et al. 2015). (2) The low-luminosity X-ray groups are confirmed to be low-mass systems through one- and two-point statistics as well as weak-lensing analysis. (3) The scaling relations between the X-ray luminosity and total group mass is verified and extended to regimes of lower masses and higher redshifts, based 


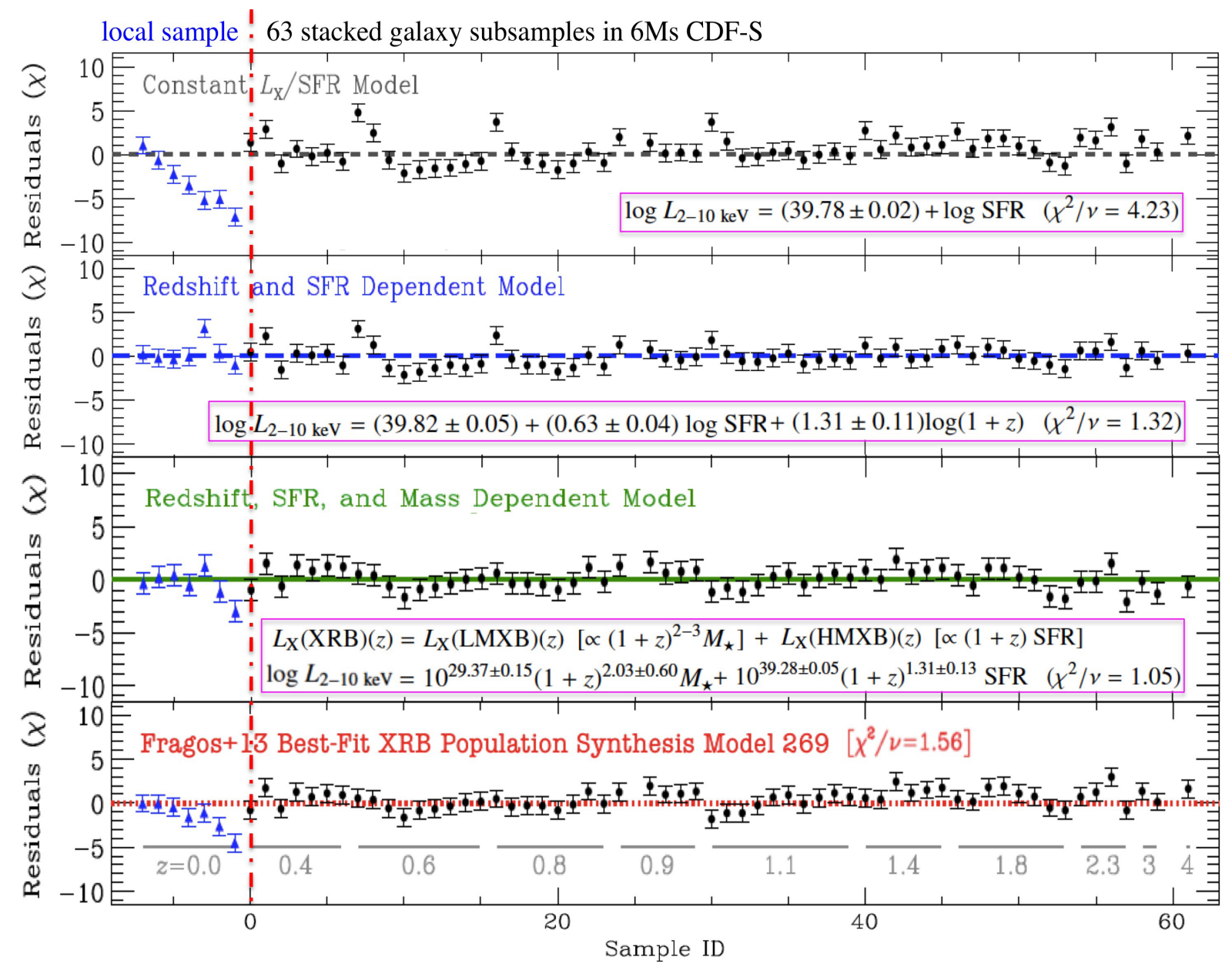

Figure 19: Fitting global non-AGN X-ray emission: residuals to model fits for a combination of the Lehmer et al. (2010) local sample (blue triangles) and 63 stacked galaxy subsamples in the 6 Ms CDF-S (black circles) using three different empirical models (with the third one providing the best characterization of $L_{X}(\mathrm{XRB})$ ) and the Fragos et al. (2013a) best-fit XRB population-synthesis model (No. 269), with model names, best-fit formula, and reduced $\chi^{2}$ values annotated. Adapted from Lehmer et al. (2016).

on stacked weak lensing and clustering analyses. These groups, with broad ranges of mass, luminosity, and redshift, make a good and representative case of groups constituting the most common environment for galaxy evolution. Moreover, there are a few notable individual structures in the E-CDF-S. For instance, the two most prominent and populated LSSs in the E-CDF-S are located at $z=0.67$ and $z=0.73$, each traced by over 60 $\mathrm{X}$-ray AGNs and many more galaxies (see the middle panel and Right-bottom panel of Fig. 20; ; and interestingly, the former LSS is in a shape of thick sheet with a radial size of $67.7 \mathrm{Mpc}$ extending over the full E-CDF-S field, and in contrast, the latter LSS is thin $(18.8 \mathrm{Mpc})$ and filamentary (e.g., Silverman et al. 2010). Another eye-catching system is the $z=1.61$ group that is the most distant X-ray group identified in the E-CDF-S and examined in detail by Tanaka et al. (2013). They find that this group is actually the lowest-mass one (with $\left.(3.2 \pm 0.8) \times 10^{13} M_{\odot}\right)$ ever confirmed at $z>1.5$, and exhibits a surprisingly prominent red sequence of quiescent early-type galaxies whose star formation is likely shut down by the bright AGN group members.

The above example works demonstrate that the CDFs are good sky patches for the census of X-ray groups and clusters, as further elucidated in the right panel of Fig. 20 that shows a number of CDF $z_{\text {spec }}$ spikes indicating (likely) LSSs, where the most up-to-date secure $z_{\text {spec }}$ compilations from Xue et al. (2016) and Luo et al. (2017) are adopted. Indeed, the CDFs are rich in LSSs: in the CDF-N, the previously known X-raysource-traced LSSs located at $z=0.12,0.46,0.63$, $0.843,1.02,1.15,2.0$, and 2.2 are all recovered by this 

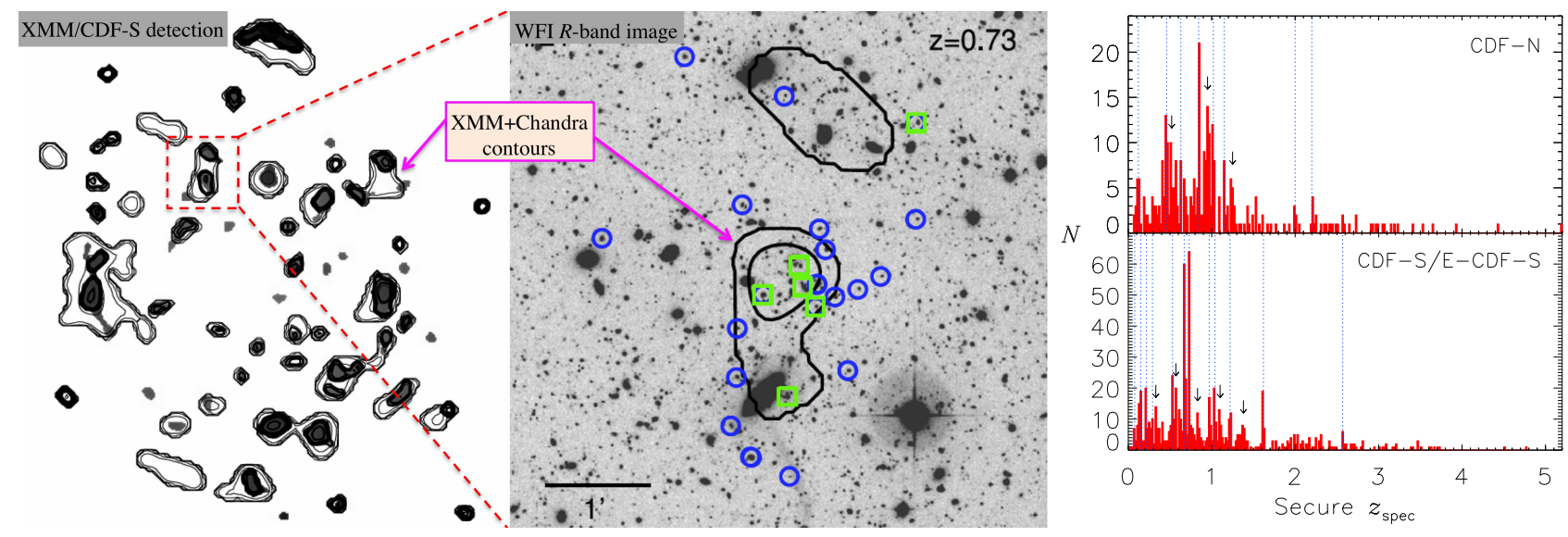

Figure 20: (Left) XMM-Newton detection of the extended emission on a 32 arcsec scale in the full E-CDF-S field, overlaid with the contours that show the extended emission detected in the combined Chandra and XMM-Newton images on the 32 and 64 arcsec scales. (Middle) The most prominent spectroscopically identified $z \approx 0.73$ group/cluster in the E-CDF-S (i.e., the highest redshift spike in the Right-bottom panel), with the contours overlaid onto the WFI $R$-band image showing the extended X-ray emission detected by XMM-Newton and Chandra. X-ray sources with $\left|z_{\text {spec }}-0.73\right|<0.01$ are labeled as circles (GOODS spectroscopy) and squares (VLT/VIMOS and Keck/DEIMOS spectroscopy). (Right) Secure $z_{\text {spec }}$ distributions of the CDF X-ray sources in bins of $\Delta z_{\text {spec }}=0.02$ (the CDF-S $z_{\text {spec }}$ 's are compiled by Luo et al. 2017, and the CDF-N and E-CDF-S $z_{\text {spec }}$ 's by Xue et al. 2016). The vertical dotted lines indicate the previously known redshift spikes (i.e., associated with LSSs) identified by X-ray sources, and the downward arrows indicate additional likely redshift spikes identified in this paper. (Left) adapted from Finoguenov et al. (2015) and (Middle) adapted from Silverman et al. (2010).

paper (indicated by dotted lines; e.g., Barger et al. 2002, 2003b; Gilli et al. 2005; Reddy et al. 2006); and in the E-CDF-S, those at $z=0.077,0.15,0.218,0.29,0.53$, $0.67,0.73,0.97,1.04,1.22,1.62$, and 2.57 are recovered by this work (e.g., Gilli et al. 2003; Treister et al. 2009c; Silverman et al. 2010; Dehghan \& JohnstonHollitt 2014). In addition to the known CDF LSSs, a total of 8 potential LSSs are identified using X-ray sources as tracers for the first time (indicated by downward arrows), which are worth further verification and quantification. Needless to say, a census of X-ray galaxy groups and clusters as complete as possible in the fields like the CDFs would be most beneficial to the improved understanding of the evolution of these systems themselves and the environmental dependence of SMBH accretion and galaxy star formation.

\section{Additional Science}

In addition to the aforementioned exciting science that can be done with the CDFs, there are furthermore a wide variety of interesting topics that can be tackled using the CDFs, e.g.: (1) Bauer et al. (2017) report a remarkable new fast X-ray transient discovered by Luo et al. (2014b) in course of the extension of CDF-S observations from $4 \mathrm{Ms}$ to $7 \mathrm{Ms}$, whose intriguing X-ray and multiwavelength properties effectively rule out the vast majority of previously known high-energy tran- sients, leaving out only a few exotic theoretical possibilities that still cannot completely explain all observed properties. The inferred rate of such events is crudely comparable to that of gamma-ray bursts, which indicates the discovery of an untapped regime for a known transient type or a new class of transient phenomenon with its nature to be determined. (2) Cappelluti et al. (2017b) report a $3 \sigma$ detection of an $\approx 3.5 \mathrm{keV}$ emission line in the CXRB spectrum derived with a combined $\approx 10 \mathrm{Ms}$ Chandra exposure of the CDF-S and COSMOS Legacy surveys, and discuss the likely origins of this observed line that include the iron line background, S XVI charge exchange, and sterile neutrino decay. (3) Using the $4 \mathrm{Ms}$ CDF-S, HST, and Spitzer data, Mitchell-Wynne et al. (2016) perform and compare the cross-correlation analyses between X-ray and optical/NIR cosmic background intensity fluctuations and find that the sources responsible for the cosmic IR background at 3.6 and $4.5 \mu \mathrm{m}$ are at least partly dissimilar to those at $\lesssim 1.6 \mu \mathrm{m}$ (also see, e.g., Cappelluti et al. 2013; Helgason et al. 2014). (4) There are a significant number of SMGs identified in the CDFs, which are of great interest given their important role in the SMBH/galaxy co-evolution picture (e.g., Alexander \& Hickox 2012; also see Section 3). Using the CDF and multiwavelength data, many topics can be studied in detail, including, e.g., the incidence of AGNs in SMGs, the location of SMGs in color-magnitude/mass 
diagrams, and coeval growth and evolutionary track of SMBHs and SMGs (e.g., Alexander et al. 2005b; Xue et al. 2010; Wardlow et al. 2011; Wang et al. 2013). (5) There is an extended X-ray emission identified in the CDF-N that is best explained as an inverse Compton ghost of a giant radio source (e.g., Fabian et al. 2009), calling for a systematic search for such sources in the CDFs. (6) There are a few tens of individual off-nuclear XRBs at $z \approx 0.05-0.3$ discovered in the CDFs, allowing for investigating their properties and redshift evolution as a population (e.g., Hornschemeier et al. 2004; Lehmer et al. 2006; also see Section 4). (7) There is a minority population of "interloping" Galactic stars in the CDFs, whose long-term evolution of magnetic activity can be studied by examining their X-ray emission (e.g., Feigelson et al. 2004).

\section{Summary and prospects}

The CDFs represent the amazing outcome of the multi-decade efforts of numerous people (counting from the proposal of Chandra submitted to NASA by R. Giacconi and H. Tananbaum in 1976 to the last CDF-S observation taken in March 2016), act as a major thrust among extragalactic X-ray surveys that are complemented effectively by deep multiwavelength observations, contribute critically to our dramatically improved characterization of the $0.5-8 \mathrm{keV}$ CXRB sources, enable a wide range of scientific topics (including AGNs, starburst and normal galaxies, groups and clusters of galaxies, LSSs, etc.), and launch literally hundreds of exciting research papers.

In this paper, I have highlighted some recent key observational results that are mostly from the CDFs and enabled by the revolutionary and versatile scientific capabilities of Chandra, including the AGN demography (see Section 2), the interactions between AGNs and their host galaxies (see Section 3), the evolution of starburst and normal galaxy X-ray emission (see Section 4), and the census of X-ray galaxy groups (see Section 5) through cosmic time. The beauty and power of the CDF treasure trove will surely be further augmented given the consistent and full exploitation of the latest CDF catalogs and products (Xue et al. 2016; Luo et al. 2017) in conjunction with the ever improving multiwavelength observations.

Despite the great advances in the above research areas, it has almost always been challenging to tell a consistent and credible story in each of these areas or regarding some of the areas as a whole, which requires reconciling/distinguishing inconsistent results (owing to, e.g., differences in sample selection criteria, analysis techniques, data qualities and depths, incompleteness levels, and/or sample sizes, as well as likely effects of cosmic variance ${ }^{13}$ and then stringing up all the reasonable pieces. Furthermore, there are many significant open questions that should persist for a foreseeable future, e.g.: (1) How to ultimately resolve the CXRB? (2) What processes dictate the formation and growth of the first SMBHs? (3) What drives the AGN downsizing behavior? (4) What are the origins of AGN variability? (5) How to obtain a census of highly obscured AGNs, CTAGNs, LLAGNs, and low-mass BHs as completely as possible? (6) What is the exact role of AGN feedback and what is the link between SMBH accretion and star formation? (7) How do accreting XRB populations evolve over most of cosmic time? (8) How do LSSs affect AGN activity? To address these questions, there are at least three aspects to move forward with each having both short-term and long-term goals associated with current and future facilities, respectively.

- Going wider. Ultradeep pencil-beam surveys such as the CDFs (see Table 1) are inevitably subject to the effects of cosmic variance. To remedy this situation and facilitate the science that requires larger solid-angle coverages, a straightforward solution is to widen the spatial coverages of the small-area surveys at moderate depths (thus being relatively easily achievable). Indeed, the CDF team has been in the process of proposing a $\approx 12 \mathrm{deg}^{2}$ Chandra/XMM-Newton survey at $30 / 50 \mathrm{ks}$ depth of the SERVS areas of WideCDF-S (W-CDF-S, centered at CDF-S), ELAIS-S1, and XMM-LSS, with 1.3 Ms of XMM-Newton observing time awarded already (PI: W. N. Brandt; see Fig. 21). These three fields all have multiple intensive radioto-UV observations performed or scheduled but lack the critical X-ray coverage. Therefore, the addition of the proposed X-ray observations will powerfully leverage those multiwavelength surveys by detecting thousands of new X-ray AGNs and hundreds of new X-ray groups/clusters, thereby dramatically advancing studies of SMBH growth across the full range of cosmic environments (from voids, groups, clusters, to the largest structures found in cold dark matter simulations), links between SMBH accretion and star formation, exceptional AGNs and protoclusters at high redshifts, and other topics. In the long run, the funded ESA-led mission Athena (band: $0.3-12 \mathrm{keV}$; large collecting area:

\footnotetext{
${ }^{13}$ Perhaps these different and even inconsistent observational manifestations highlight, to some degree, the vast complexities and personalities of galaxies and AGNs that essentially preclude any simple "universal" scenarios regarding many questions about them.
} 
$\approx 2.0-2.5 \mathrm{~m}^{2}$; large field of view: $\approx 40 \times 40$ arcmin; good angular resolution: $\approx 3-5$ arcsec; scheduled launch: 2028; Nandra et al. 2013) will be $\approx 100$ times more efficient in carrying out deep and wide surveys than Chandra and XMM-Newton for a given combination of solid-angle coverage and flux limit (above $f_{\mathrm{SB}} \gtrsim(1-2) \times 10^{-17} \mathrm{erg} \mathrm{cm}^{-2} \mathrm{~s}^{-1}$, which corresponds to Athena's source confusion limit and sensitivity limits of $\approx 2$ Ms Chandra exposures; see Table 11, thus substantially revolutionizing extragalactic X-ray studies. A large strategic mission concept, Lynx (called X-ray Surveyor previously; e.g., Weisskopf et al. 2015), is essentially to build a super Chandra with significantly enlarged collecting area (thus tremendously increased sensitivities) and still sub-arcsecond angular resolution; if selected and funded, it will take the sharpest X-ray vision even fainter and farther, thereby prodigiously transforming the field as Athena would do.

- Going harder. Sensitive hard X-ray ( $\gtrsim 10 \mathrm{keV})$ surveys can open up a large volume of discovery space uncharted by Chandra and XMM-Newton, deciphering the even more energetic universe. Deep hard X-ray observations that fully encompass the CXRB peak can provide additional critical insights into the origin of the CXRB and unveil the highly obscured and CT AGNs. In this regard, NUSTAR, as the first focusing hard X-ray observatory in orbit, has been a pathbreaker that is significantly influencing the field. NUSTAR has already observed the CDFs with 200 ks depth (see Footnotes 2 and 3), being able to detect Seyfert-like sources $\left(L_{\mathrm{X}}<10^{44} \mathrm{erg} \mathrm{s}^{-1}\right)$ up to $z \approx 0.5$. Future more sensitive hard X-ray observations would be expected from $H E X$ $P$ (PI: F. Harrison) if funded, which has been proposed to be a natural successor to NUSTAR.

- Going more. Additional sky coverages at the CDF flux levels are critically important as they substantially improve the statistical sample sizes of the faintest X-ray sources and also allow for a basic assessment of the effects of cosmic variance. It is well expected that the CDF-like surveys will become commonplace once the next-generation large X-ray observatories such as Athena and Lynx are put into operation. Specifically, Lynx will have the capability of efficiently performing even deeper surveys than the CDFs, thus effectively going deeper and wider simultaneously (see the above Going wider point).

\section{Acknowledgements:}

I thank the referee for careful reading and constructive comments that helped improve this review. I thank
W.N. Brandt "who led the effort to obtain the deepest Chandra fields" (excerpted from the citation of his 2016 Bruno Rossi Prize), thank B.D. Lehmer for producing Figures 4, 5, and 6 exclusively for this paper, thank B. Luo for providing Figure 21, and thank G. Yang for making Figure 8. I thank D.M. Alexander, F.E. Bauer, W.N. Brandt, B.D. Lehmer, B. Luo, M.Y. Sun, F. Vito, and G. Yang for helpful feedback, discussions, and information sharing, as well as consistent collaboration. I also thank all my other collaborators for working on various projects together. The following funding support is gratefully acknowledged: the 973 Program (2015CB857004), the CAS Frontier Science Key Research Program (QYZDJ-SSWSLH006), the National Natural Science Foundation of China (NSFC-11473026, 11421303), the Fundamental Research Funds for the Central Universities, the Thousand Young Talents Plan of China, and the CAS Strategic Priority Research Program "The Emergence of Cosmological Structures” (XDB09000000).

\section{References}

Abdo, A. A. et al., 2011. ApJL 733, 26.

Akiyama, M., 2005. ApJ 629, 72.

Alexander, D. M. et al., 2003. AJ 126, 539.

Alexander, D. M., Bauer, F. E., Chapman, S. C., Smail, I., Blain, A. W., Brandt, W. N., Ivison, R. J., 2005a. ApJ 632, 736.

Alexander, D. M., Smail, I., Bauer, F. E., Chapman, S. C., Blain, A. W., Brandt, W. N., Ivison, R. J., 2005b. Nature 434, 738.

Alexander, D. M. et al., 2008a. ApJ 687, 835.

Alexander, D. M. et al., 2008b. AJ 135, 1968.

Alexander, D. M. et al., 2011. ApJ 738, 44.

Alexander, D. M., Hickox, R. C., 2012. NewAR 56, 93.

Alexander, D. M. et al., 2013. ApJ 773, 125.

Allen, S. W., Evrard, A. E., Mantz, A. B., 2011. ARA\&A 49, 409.

Almaini, O. et al., 2000. MNRAS 315, 325.

Alonso-Herrero, A. et al., 2008. ApJ 677, 127.

Aird, J. et al., 2010. MNRAS 401, 2531.

Aird, J. et al., 2012. ApJ 746, 90.

Aird, J. et al., 2013. ApJ 775, 41.

Aird, J. et al., 2015a. ApJ 815, 66.

Aird, J. et al., 2015b. MNRAS 451, 1892.

Aird, J., Coil, A. L., Georgakakis, A., 2017. MNRAS 465, 3390.

Akylas, A., Georgantopoulos, I., Ranalli, P., Gkiokas, E., Corral, A., Lanzuisi, G., 2016. A\&A 594, 73.

Andreon, S., Maughan, B., Trinchieri, G., Kurk, J., 2009. A\&A 507, 147.

Ashby, M. L. N. et al., 2013. ApJ 769, 80.

Assef, R. J. et al., 2015. ApJ 804, 27.

Azadi, M. et al., 2015. ApJ 806, 187.

Azadi, M. et al., 2017. ApJ 835, 27.

Babić, A., Miller, L., Jarvis, M. J., Turner, T. J., Alexander, D. M., Croom, S. M., 2007. A\&A 474, 755.

Baldassare, V. F., Reines, A. E., Gallo, E., Greene, J. E., 2015. ApJL $809,14$.

Baldassare, V. F., Reines, A. E., Gallo, E., Greene, J. E., 2017. ApJ 836, 20.

Ballantyne, D. R. et al., 2006. ApJ 653, 1070. 

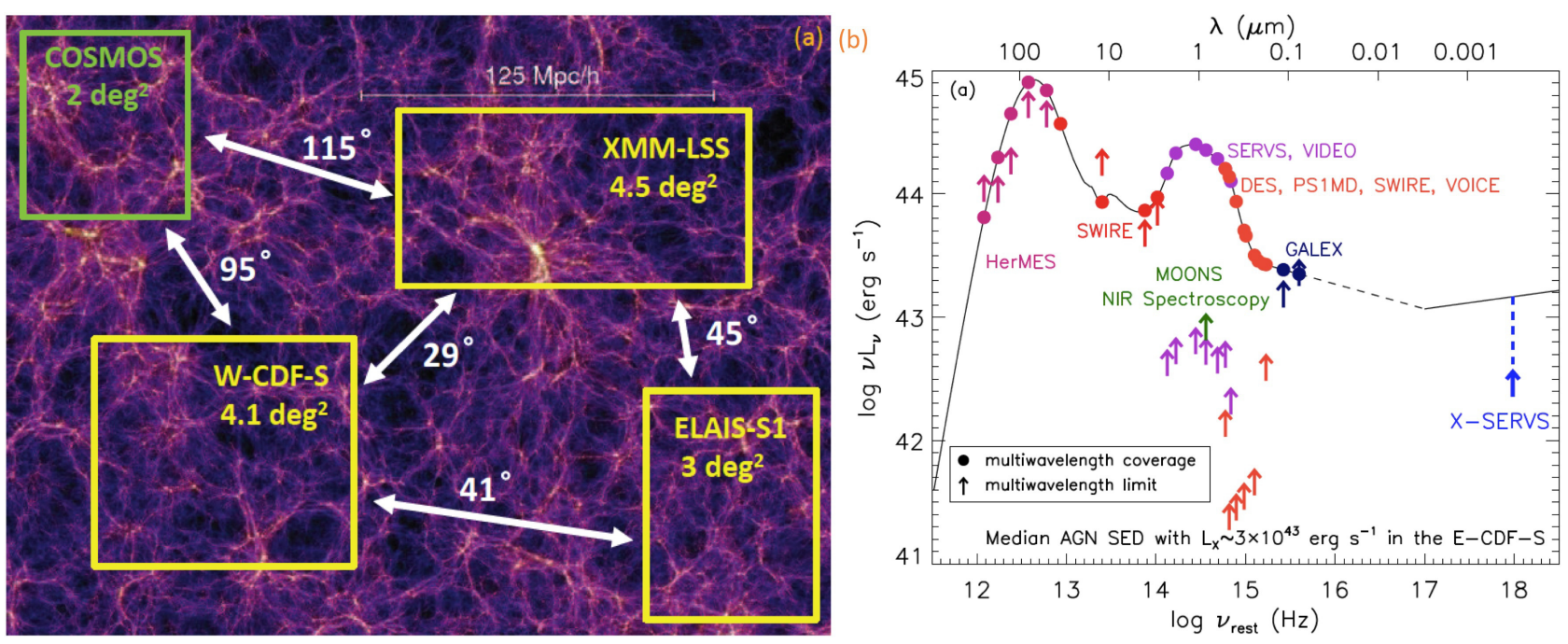

Figure 21: (Left) Projected density map at $z=1.4$ with comoving size $350 \times 450 \mathrm{Mpc}^{2}$ from the Millennium Simulation (Springel et al. 2005), with the sizes of COSMOS and the porposed X-SERVS fields as well as their respective separations annotated. (Right) Median SED of AGNs with $L_{\mathrm{X}} \approx 3 \times 10^{43} \mathrm{erg} \mathrm{s}^{-1}$ in the E-CDF-S combined with a composite FIR starburst galaxy SED (Sajina et al. 2012) at $z=1$. The data points and upward arrows indicate the current/scheduled X-SERVS multiwavelength coverage and their $z=1$ limiting luminosities; and the rightmost blue arrow indicates the proposed critical X-ray coverage that is currently missing (courtesy of B. Luo).

Ballantyne, D. R., Draper, A. R., Madsen, K. K., Rigby, J. R., Treister, E., 2011. ApJ 736, 56.

Ballo, L. et al., 2007. ApJ 667, 97.

Barger, A. J. et al., 2002. AJ 124, 1839.

Barger, A. J., Cowie, L. L., Capak, P., Alexander, D. M., Bauer, F. E., Brandt, W. N., Garmire, G. P., Hornschemeier, A. E., 2003a. ApJL 584,61 .

Barger, A. J. et al., 2003b. AJ 126, 632.

Barger, A. J., Cowie, L. L., Mushotzky, R. F., Yang, Y., Wang, W. H., Steffen, A. T., Capak, P., 2005. AJ 129, 578.

Barger, A. J., Cowie, L. L., Owen, F. N., Chen, C.-C., Hasinger, G., Hsu, L.-Y., Li, Y., 2015. ApJ 801, 87.

Basu-Zych, A. R. et al., 2013. ApJ 762, 45.

Bauer, F. E., Alexander, D. M., Brandt, W. N., Hornschemeier, A. E., Vignali, C., Garmire, G. P., Schneider, D. P., 2002a. AJ 124, 2351. Bauer, F. E. et al., 2002b. AJ 123, 1163.

Bauer, F. E., Alexander, D. M., Brandt, W. N., Schneider, D. P., Treister, E., Hornschemeier, A. E., Garmire, G. P., 2004. AJ 128, 2048 Bauer, F. E. et al., 2017. MNRAS 467, 4841.

Beckwith, S. V. W. et al., 2006. AJ 132, 1729.

Bell, E. F. et al., 2004. ApJ 608, 752.

Bennert, V. N., Auger, M. W., Treu, T., Woo, J.-H., Malkan, M. A., 2011. ApJ 742, 107.

Bernhard, E., Mullaney, J. R., Daddi, E., Ciesla, L., Schreiber, C., 2016. MNRAS 460, 902.

Bielby, R. M. et al., 2010. A\&A 523, 66.

Boller, Th., Freyberg, M. J., Trümper, J., Haberl, F., Voges, W., Nandra, K., 2016. A\&A 588, 103.

Bonfield, D. G. et al., 2011. MNRAS 416, 13.

Bongiorno, A. et al., 2012. MNRAS 427, 3103.

Bongiorno, A. et al., 2014. MNRAS 443, 2077.

Bonzini, M. et al., 2012. ApJS 203, 15.

Booth, C. M., Schaye, J., 2009. MNRAS 398, 53.

Boroson, B., Kim, D.-W., Fabbiano, G., 2011. ApJ 729, 12.

Borys, C., Smail, Ian, Chapman, S. C., Blain, A. W., Alexander, D.

M., Ivison, R. J., 2005. ApJ 635, 853.
Boutsia, K., Leibundgut, B., Trevese, D., Vagnetti, F., 2009. A\&A 497, 81.

Brammer, G. B. et al., 2009. ApJL 706, 173.

Brandt, W. N. et al., 2001. AJ 122, 2810.

Brandt, W. N., Alexander, D. M., 2010. PNAS 107, 7184.

Brandt, W. N., Alexander, D. M., 2015. A\&A Rev. 23, 1.

Brandt, W. N., Hasinger, G., 2005. ARA\&A 43, 827.

Brandt, W. N., Vito, F., 2017. AN 338, 241.

Brightman, M., Nandra, K., 2011. MNRAS 413, 1206.

Brightman, M., Nandra, K., Salvato, M., Hsu, L. T., Aird, J., Rangel, C., 2014. MNRAS 443, 1999.

Brightman, M., Ueda, Y., 2012. MNRAS 423, 702.

Brightman, M. et al., 2013. MNRAS 433, 2485.

Brightman, M. et al., 2015. ApJ 805, 41.

Broos, P. S. et al., 2010. ApJ 714, 1582.

Brusa, M., et al., 2005. A\&A 432, 69.

Brusa, M., et al., 2007. ApJS 172, 353.

Brusa, M. et al., 2009a. ApJ 693, 8.

Brusa, M. et al., 2009b. A\&A 507, 1277.

Buchner, J. et al., 2014. A\&A 564, 125.

Buchner, J. et al., 2015. ApJ 802, 89.

Buchner, J., Bauer, F. E., 2017. MNRAS 465, 4348.

Bundy, K., Ellis, R. S., Conselice, C. J., 2005. ApJ 625, 621.

Burlon, D. et al., 2011. ApJ 728, 58.

Cai, Z.-Y., Wang, J.-X., Gu, W.-M., Sun, Y.-H., Wu, M.-C., Huang, X.-X., Chen, X.-Y., 2016. ApJ 826, 7.

Caldwell, J. A. R. et al., 2008. ApJS 174, 136.

Capak, P. et al., 2004. AJ 127, 180.

Cappelluti, N. et al., 2009. A\&A 497, 635.

Cappelluti, N. et al., 2013. ApJ 769, 68.

Cappelluti, N. et al., 2016. ApJ 823, 95.

Cappelluti, N. et al., 2017. ApJ 837, 19.

Cappelluti, N. et al., submitted. ApJ < arXiv:1701.07932 >

Cardamone, C. N. et al., 2010. ApJS 189, 270.

Cattaneo, A. et al., 2009. Nature 460, 213.

Chang, Y.-Y. et al., 2017. MNRAS 466, L103 
Chen, C.-T. J. et al., 2013. ApJ 773, 3.

Chen, C.-T. J. et al., 2017. ApJ 837, 48.

Chiappetti, L. et al., 2005. A\&A 439, 413.

Chiappetti, L. et al., 2013. MNRAS 429, 1652.

Civano, F. et al., 2011. ApJ 741, 91

Civano, F. et al., 2016. ApJ 819, 62

Colbert, E. J. M., Heckman, T. M., Ptak, A. F., Strickland, D. K., Weaver, K. A., 2004. ApJ 602, 231.

Comastri, A., 2014. ASSL 308, 245.

Comastri, A. et al., 2011. A\&A 526, L9.

Connolly, S. D. et al., 2016. MNRAS 459, 3963.

Coppin, K. E. K. et al., 2009. MNRAS 395, 1905.

Cotini, S., Ripamonti, E., Caccianiga, A., Colpi, M., Mapelli, M., Severgnini, P., Segreto, A., 2013. MNRAS 431, 2661.

Cowie, L. L., Barger, A. J., Bautz, M. W., Brandt, W. N., Garmire, G. P., 2003. ApJL 584, 57.

Cowie, L. L., Barger, A. J., Hasinger, G., 2012. ApJ 748, 50.

Cristiani, S. et al., 2004. ApJL 600, 119.

Daddi, E., et al., 2007. ApJ 670, 173.

Damen, M. et al., 2011. ApJ 727, 1.

Danielson, A. L. R., Lehmer, B. D., Alexander, D. M., Brandt, W. N., Luo, B., Miller, N., Xue, Y. Q., Stott, J. P., 2012. MNRAS 422, 494

De Luca, A., Molendi, S., 2004. A\&A 419, 837.

De Lucia, G., Springel, V., White, S. D. M., Croton. D., Kauffmann. G., 2006. MNRAS 366, 499.

Dehghan, S., Johnston-Hollitt, M., 2014. AJ 147, 52.

Del Moro, A. et al., 2013. A\&A 549, 59.

Del Moro, A. et al., 2014. ApJ 786, 16.

Del Moro, A. et al., 2016. MNRAS 456, 2105.

Delvecchio, I. et al., 2015. MNRAS 449, 373.

den Brok, M. et al., 2015. ApJ 809, 101.

Di Matteo, T., Springel, V., Hernquist, L., 2005. Nature 433, 604.

Di Matteo, T., Colberg, J., Springel, V., Hernquist, L., Sijacki, D., 2008. ApJ 676, 33.

Diamond-Stanic, A. M., Rieke, G. H., 2012. ApJ 746, 168

Dong, X.-B. et al., 2012. ApJ 755, 167.

Dong, X. Y., Wu, X.-B., 2016. ApJ 824, 70.

Dwelly, T., Page, M. J., 2006. MNRAS 372, 1755.

Ehlert, S., Allen, S. W., Brandt, W. N., Xue, Y. Q., Luo, B., von der Linden, A., Mantz, A., Morris, R. G., 2013. MNRAS 428, 3509.

Ehlert, S. et al., 2014. MNRAS 437, 1942.

Ehlert, S. et al., 2015. MNRAS 446, 2709.

Elbaz, D. et al., 2011. A\&A 533, 119.

Emmanoulopoulos, D. et al., 2012. MNRAS 424, 1327.

Fabbiano, G., 2006. ARA\&A 44, 323.

Fabian, A. C., 2012. ARA\&A 50, 455.

Fabian, A. C., Chapman, S., Casey, C. M., Bauer, F., Blundell, K. M., 2009. MNRAS 395, L67.

Fabian, A. C., Vasudevan, R. V., Gandhi, P., 2008. MNRAS 385, L43.

Fan, L., Fang, G., Chen, Y., Li, J., Lv, X., Knudsen, K. K., Kong, X., 2014. ApJL 784, 9.

Fanali, R. et al., 2013. MNRAS 433, 648.

Fausnaugh, M. M. et al., 2016. ApJ 821, 56.

Feigelson, E. D. et al., 2004. ApJ 611, 1107.

Ferrarese, L., Ford, H., 2005. SSRv 116, 523.

Ferrarese, L., Merritt, D., 2000. ApJL 539, 9.

Filiz Ak, N. et al., 2013. ApJ 777, 168.

Finoguenov, A. et al., 2006. ApJ 646, 143.

Finoguenov, A. et al., 2007. ApJS 172, 182.

Finoguenov, A. et al., 2009. ApJ 704, 564.

Finoguenov, A. et al., 2010. MNRAS 403, 2063.

Finoguenov, A. et al., 2015. A\&A 576, 130.

Fiore, F. et al., 2008. ApJ 672, 94

Fiore, F. et al., 2009. ApJ 693, 447.
Fiore, F., Puccetti, S., Mathur, S., 2012. AdAst 2012, 9.

Fiore, F. et al., 2012. A\&A 537, 16

Falocco, S. et al., 2015. A\&A 579, 115.

Fotopoulou, S. et al., 2016. A\&A 587, 142.

Fragos, T. et al., 2013a. ApJ 764, 41.

Fragos, T., Lehmer, B. D., Naoz, S., Zezas, A., Basu-Zych, A., 2013 b. ApJL 776, 31.

Gabor, J. M., Bournaud, F., 2013. MNRAS 434, 606.

Garmire, G. P., Bautz, M. W., Ford, P. G., Nousek, J. A., Ricker, Jr., G. R., 2003. Proc. SPIE 4851, 28.

Geach, J. E. et al., 2017. MNRAS 465, 1789.

Gebhardt, K. et al., 2000. ApJL 539, 13.

Gebhardt, K. et al., 2001. AJ 122, 2469.

Georgakakis, A., et al., 2006. MNRAS 367, 1017.

Georgakakis, A., Nandra, K., Laird, E. S., Aird, J., Trichas, M., 2008. MNRAS 388, 1205.

Georgakakis, A., et al., 2015. MNRAS 453, 1946.

Georgantopoulos, I., Akylas, A., Georgakakis, A., Rowan-Robinson, M., 2009. A\&A 507, 747.

Georgantopoulos, I. et al., 2011a. A\&A 531, 116.

Georgantopoulos, I. et al., 2011b. A\&A 534, 23.

Georgantopoulos, I., 2013. IJMPS. 23, 1.

Georgantopoulos, I. et al., 2013. A\&A 555, 43.

Ghosh, H., Mathur, S., Fiore, F., Ferrarese, L., 2008. ApJ 687, 216.

Giacconi, R. et al., 1962. PRL 9, 439.

Giacconi, R. et al., 2002. ApJS 139, 369

Giallongo, E., Menci, N., Fiore, F., Castellano, M., Fontana, A., Grazian, A., Pentericci, L., 2012. ApJ 755, 124.

Giallongo, E. et al., 2015. A\&A 578, 83.

Giavalisco, M. et al., 2004. ApJL 600, L93.

Gilli, R. et al., 2003. ApJ 592, 721.

Gilli, R., 2004. AdSpR 34, 2470.

Gilli, R. et al., 2005. A\&A 430, 811.

Gilli, R., Comastri, A., Hasinger, G., 2007. A\&A 463, 79.

Gilli, R., Vignali, C., Mignoli, M., Iwasawa, K., Comastri, A., Zamorani, G., 2010. A\&A 519, 92.

Gilli, R. et al., 2011. MSAIS 17, 85.

Gilli, R. et al., 2011. ApJL 730, 28.

Gilli, R., 2013. MmSAI 84, 647.

Gilli, R. et al., 2014. A\&A 562, 67.

Gobat, R. et al., 2011. A\&A 526, 133.

Constantin, A. et al., 2009. ApJ 705, 1336.

González-Martín, O., Vaughan, S., 2012. A\&A 544, 80.

Goulding, A. D. et al., 2012. ApJ 755, 5.

Graham, A. W., Scott, N., 2013. ApJ 764, 151.

Graham, A. W., Scott, N., 2015. ApJ 798, 54.

Granato, G. L., Silva, L., Lapi, A., Shankar, F., De Zotti, G., Danese, L., 2006. MNRAS 368, L72.

Grazian, A. et al., 2006. A\&A 449, 951.

Greene, J. E., 2012. NatCo 3, 1304.

Greene, J. E. et al., 2010. ApJ 721, 26.

Greene, J. E., Ho, L. C., 2004. ApJ 610, 722.

Grogin, N. A. et al., 2005. ApJL 627, 97.

Grogin, N. A. et al., 2011. ApJS 197, 35

Grissom, R. L., Ballantyne, D. R., Wise, J. H., 2014. A\&A 561, 90.

Gu, M., Cao, X., 2009. MNRAS 399, 349.

Gültekin, K. et al., 2009. ApJ 698, 198.

Haardt, F., Madau, P., 2012. ApJ 746, 125.

Haines, C. P. et al., 2012. ApJ 754, 97.

Hainline, K. N., Reines, A. E., Greene, J. E., Stern, D., 2016. ApJ $832,119$.

Häring, N., Rix, H.-W., 2004. ApJL 604, 89.

Harris, K. et al., 2016. MNRAS 457, 4179.

Harrison, C., 2017. NatAs 1, 165.

Harrison, C. M. et al., 2012. ApJL 760, 15. 
Harrison, F. A. et al., 2016. ApJ 831, 185.

Hasinger, G., 2008. A\&A 490, 905.

Hasinger, G., Miyaji, T., Schmidt, M., 2005. A\&A 441, 417.

Hatziminaoglou, E. et al., 2010. A\&A 518, L33.

Kauffmann, G., Heckman, T. M., 2009. MNRAS 397, 135

Habouzit, M., Volonteri, M., Latif, M., Dubois, Y., Peirani, S., 2016. MNRAS 463, 529.

Heckman, T. M. et al., 2004. ApJ 613, 109.

Heckman, T. M., Best, P. N., 2014. ARA\&A 52, 589.

Helgason, K., Cappelluti, N., Hasinger, G., Kashlinsky, A., Ricotti, M., 2014. ApJ 785, 38.

Henry, J. P., Mullis, C. R., Voges, W., Böhringer, H., Briel, U. G., Gioia, I. M., Huchra, J. P., 2006. ApJS 162, 304.

Henry, J. P. et al., 2010. ApJ 725, 615

Hernán-Caballero, A. et al., 2014. MNRAS 443, 3538.

Hickox, R. C., et al., 2009. ApJ 696, 891.

Hickox, R. C., et al., 2014. ApJ 782, 9.

Hickox, R. C., Markevitch, M., 2006. ApJ 645, 95.

Hiroi, K., Ueda, Y., Akiyama, M., Watson, M. G., 2012. ApJ 758, 49 Hirschmann, M. et al., 2010. MNRAS 407, 1016.

Ho, L. C., 2007. ApJ 668, 94.

Ho, L. C., Kim, M., 2016. ApJ 821, 48.

Hodge, J. A. et al., 2013. ApJ 768, 91.

Hopkins, P. F., Hernquist, L., 2006. ApJS 166, 1.

Hopkins, P. F., Hernquist, L., Cox, T. J., Di Matteo, T., Robertson, B., Springel, V., 2006a. ApJS 163, 1.

Hopkins, P. F. et al., 2006b. ApJ 652, 864.

Hopkins, P. F., Hernquist, L., Cox, T. J., Keres, D., 2008. ApJS 175, 356.

Hopkins, P. F., Quataert, E., 2010. MNRAS 407, 1529.

Hornschemeier, A. E. et al., 2004. ApJL 600, 147.

Hornschemeier, A. E., Heckman, T. M., Ptak, A. F., Tremonti, C. A., Colbert, E. J. M., 2005. AJ 129, 86.

Hsieh, B.-C., Wang, W.-H., Yan, H. J., Lin, L. W., Karoji, H., Lim, J., Ho, P. T. P., Tsai, C.-W., 2012. ApJ 749, 88.

Hsu, L.-T. et al., 2014. ApJ 796, 60.

Ishida, M. et al., 2011. PASJ 63S, 657.

Iwasawa, K. et al., 2012. A\&A 546, 84.

Jahnke, K. et al., 2009. ApJL 706, 215.

Jahnke, K., Macciò, A. V., 2011. ApJ 734, 92.

Jang, I. et al., 2014. MNRAS 443, 72.

Jardel, J. R., Gebhardt, K., 2012. ApJ 746, 89.

Jia, J. et al., 2011. ApJ 731, 55

Jiang, Y.-F., Greene, J. E., Ho, L. C., 2011. ApJL 737, 45.

Jones, M. L., Hickox, R. C., Black, C. S., Hainline, K. N., DiPompeo, M. A., Goulding, A. D., 2016. ApJ 826, 12.

Kalfountzou, E., Civano, F., Elvis, M., Trichas, M., Green, P., 2014 MNRAS 445, 1430.

Kamizasa, N., Terashima, Y., Awaki, H., 2012. ApJ 751, 39.

Kauffmann, G., 1996. MNRAS 281, 487.

Kauffmann, G., Heckman, T. M., 2009. MNRAS 397, 135.

Kawamuro, T. et al., 2016. ApJ 831, 37.

Kelly, B. C., Bechtold, J., Siemiginowska, A., 2009. ApJ 698, 895.

Kelly, B. C., Treu, T., Malkan, M., Pancoast, A., Woo, J.-H., 2013. ApJ 779, 187.

Kelly, B. C., Becker, A. C., Sobolewska, M., Siemiginowska, A., Uttley, P., 2014. ApJ 788, 33.

Kim, M., Wilkes, B. J., Kim, D.-W., Green, P. J., Barkhouse, W. A., Lee, M. G., Silverman, J. D., Tananbaum, H. D., 2007. ApJ 659, 29.

Kiziltan, B., Baumgardt, H., Loeb, A., 2017. Nature 542, 203.

Kocevski, D. D., et al., 2012. ApJ 744, 148.

Kocevski, D. D., et al., 2015. ApJ 814, 104

Koekemoer, A. M. et al., 2011. ApJS 197, 36

Kollmeier, J. A. et al., 2006, ApJ 648, 128.
Kormendy, J., Gebhardt, K., 2001. AIPC 586, 363.

Kormendy, J., Ho, L. C., 2013. ARA\&A 51, 511.

Kormendy, J., Richstone, D., 1995. ARA\&A 33, 581.

Koshida, S. et al., 2014. ApJ 788, 159.

Koss, M. J. et al., 2016. ApJ 825, 85.

Koss, M., Mushotzky, R., Veilleux, S., Winter, L., 2010. ApJL 716, 125.

Koulouridis, E. et al., 2014. A\&A 567, 83.

Koulouridis, E. et al., 2016. A\&A 592, 11.

Kriek, M. et al., 2008. ApJ 677, 219.

La Franca, F. et al., 2005. ApJ 635, 864.

Laird, E. S. et al., 2006. MNRAS 373, 217.

Laird, E. S. et al., 2009. ApJS 180, 102

LaMassa, S. M. et al., 2015. ApJ 800, 144.

LaMassa, S. M. et al., 2016. ApJ 817, 172.

Lanzuisi, G. et al., 2013. MNRAS 431, 978.

Lanzuisi, G. et al., 2014. ApJ 781, 105.

Lanzuisi, G. et al., 2015a. A\&A 573, 137.

Lanzuisi, G. et al., 2015b. A\&A 578, 120

Lanzuisi, G. et al., 2017. A\&A 602, 123.

Lauer, T. R., Tremaine, S., Richstone, D., Faber, S. M., 2007. ApJ $670,249$.

Lehmann, I. et al., 2001. A\&A 371, 833.

Lehmer, B. D., et al., 2005. ApJS 161, 21.

Lehmer, B. D., et al., 2006. AJ 131, 2394.

Lehmer, B. D., et al., 2007. ApJ 657, 681.

Lehmer, B. D., et al., 2008. ApJ 681, 1163.

Lehmer, B. D. et al., 2009. MNRAS 400, 299.

Lehmer, B. D., et al., 2010. ApJ 724, 559.

Lehmer, B. D. et al., 2012. ApJ 752, 46.

Lehmer, B. D., et al., 2014. ApJ 789, 52.

Lehmer, B. D. et al., 2016. ApJ 825, 7.

Lemons, S. M., Reines, A. E., Plotkin, R. M., Gallo, E., Greene, J. E., 2015. ApJ 805, 12

Liu, H., Li, S.-L., Gu, M., Guo, H., 2016a. MNRAS 462, L56.

Liu, T. et al., 2015. ApJS 216, 28

Liu, T. et al., 2017. ApJS 232, 8.

Liu, Z. et al., 2016b. MNRAS 459, 1602.

Luo, B. et al., 2008. ApJS 119, 19.

Luo, B. et al., 2010. ApJS 187, 560

Luo, B. et al., 2011. ApJ 740, 37.

Luo, B. et al., 2014a. ApJ 794, 70.

Luo, B., Brandt, N., Bauer, F., 2014b. ATel 6541, 1.

Luo, B. et al., 2017. ApJS 228, 2.

Lora, V., Sánchez-Salcedo, F. J., Raga, A. C., Esquivel, A., 2009 ApJL 699, 113

Lusso, E. et al., 2012. MNRAS 425, 623.

Lusso, E. et al., 2013. ApJ 777, 86.

Lutz, D. et al., 2008. ApJ 684, 853.

Lutz, D. et al., 2010. ApJ 712, 1287.

Lutz, D. et al., 2011. A\&A 532, 90.

Lutz, D., Maiolino, R., Spoon, H. W. W., Moorwood, A. F. M., 2004. A\&A 418, 465.

MacLeod, C. L. et al., 2011. ApJ 728, 26.

Madau, P., Dickinson, M., 2014. ARA\&A 52, 415.

Madsen, K. K., Beardmore, A. P., Forster, K., Guainazzi, M., Marshall, H. L., Miller, E. D., Page, K. L., Stuhlinger, M., 2017. AJ $153,2$.

Magorrian, J. et al., 1998. AJ 115, 2285.

Maiolino, R. et al., 1998. A\&A 338, 781.

Maiolino, R. et al., 2017. Nature 544, 202.

Malizia, A. et al., 2009. MNRAS 399, 944.

Manners, J. C. et al., 2003. MNRAS 343, 293.

Maoz, D., Nagar, N. M., Falcke, H., Wilson, A. S., 2005. ApJ 625, 699. 
Marchesi, S. et al., 2016. ApJ 827, 150.

Marconi, A., Hunt, L. K., 2003. ApJL 589. 21.

Martini, P. et al., 2013. ApJ 768, 1.

Marulli, F., Bonoli, S., Branchini, E., Moscardini, L., Springel, V., 2008. MNRAS 385, 1846.

Mateos, S. et al., 2007. A\&A 473, 105.

Mateos, S. et al., 2015. MNRAS 449, 1422.

Matsuoka, K. et al., 2013. ApJ 771, 64.

Matsuoka, K., Woo, J.-H., 2015. ApJ 807, 28.

Matt, G., Fabian, A. C., Guainazzi, M., Iwasawa, K., Bassani, L., Malaguti, G., 2000. MNRAS 318, 173.

Matt, G., Guainazzi, M., Maiolino, R., 2003. MNRAS 342, 422.

McConnell, N. J., Ma, C.-P., 2013. ApJ 764, 184.

McElroy, R. E. et al., 2016. A\&A, 593, L8.

McHardy, I. M. et al., 2013. MNRAS 430, L49.

McHardy, I. M., Papadakis, I. E., Uttley, P., Page, M. J., Mason, K. O., 2004. MNRAS 348, 783.

McLure, R. J., Dunlop, J. S., 2002. MNRAS 331, 795.

McNamara, B. R., Nulsen, P. E. J., 2012. NJPh 14, 5023.

Melnyk, O. et al. 2013. A\&A 557, 81.

Menci, N., Fiore, F., Puccetti S., Cavaliere, A., 2008. ApJ 686, 219.

Merloni, A. et al., 2010. ApJ 708, 137.

Merloni, A. et al., 2012. < arXiv:1209.3114>.

Merloni, A. et al., 2014. MNRAS 437, 3550.

Merloni, A., Rudnick, G., Di Matteo, T., 2004. MNRAS 354, L37.

Mezcua, M., Civano, F., Fabbiano, G., Miyaji, T., Marchesi, S., 2016. ApJ. 817, 20.

Middei, R., Vagnetti, F., Bianchi, S., La Franca, F., Paolillo, M., Ursini, F., 2017. A\&A, 599, 82.

Mignoli, M. et al., 2013. A\&A 556, 29

Miller, B. P. et al., 2015. ApJ 799, 98.

iller, M. C., Colbert, E. J. M., 2004. IJMPD 13, 1.

Miller, N. A. et al., 2013. ApJS 205, 13

Mineo, S., Gilfanov, M., Sunyaev, R., 2012. MNRAS 419, 2095.

Mineo, S., Gilfanov, M., Lehmer, B. D., Morrison, G. E., Sunyaev, R., 2014. MNRAS 437, 1698 .

Miniati, F., Finoguenov, A., Silverman, J. D., Carollo, M., Cibinel, A., Lilly, S. J., Schawinski, K., 2016. ApJ 819, 26.

Mitchell-Wynne, K., Cooray, A., Xue, Y. Q., Luo, B., Brandt, W., Koekemoer, A., 2016. ApJ 832, 104.

Miyaji, T., Hasinger, G., Schmidt, M., 2000. A\&A 353, 25.

Miyaji, T., Hasinger, G., Schmidt, M., 2001. A\&A 369, 49.

Miyaji, T., Griffiths, R. E., Lumb, D., Sarajedini, V., Siddiqui, H., 2003. AN 324, 24.

Miyaji, T. et al., 2015. ApJ 804, 104.

Moran, E. C., Shahinyan, K., Sugarman, H. R., Vélez, D. O., Eracleous, M., 2014. AJ 148, 136.

Moretti, A., Campana, S., Lazzati, D., Tagliaferri, G., 2003. ApJ 588, 696.

Moretti, A., et al., 2009. A\&A 493, 501.

Moretti, A., Vattakunnel, S., Tozzi, P., Salvaterra, R., Severgnini, P., Fugazza, D., Haardt, F., Gilli, R., 2012. A\&A 548, 87.

Morrison, G. E., Owen, F. N., Dickinson, M., Ivison, R. J., Ibar, E., 2010. ApJS 188, 178 .

Morrissey, P. et al., 2007. ApJS 173, 682.

ullaney, J. R., Alexander, D. M., Goulding, A. D., Hickox R. C., 2011. MNRAS 414, 1082

Mullaney, J. R. et al., 2012a. MNRAS 419, 95.

Mullaney, J. R. et al., 2012b. ApJL 753, 30.

Mullaney, J. R. et al., 2015a, ApJ 808, 184.

Mullaney, J. R. et al., 2015b, MNRAS 453, L83.

Murphy, K. D., Yaqoob, T., 2009. MNRAS 397, 1549.

Murray, S. S. et al., 2005. ApJS 161, 1.

Mushotzky, R. F., Done, C., Pounds, K. A., 1993. ARA\&A 31, 717.

Neistein, E., Netzer, H., 2014. MNRAS 437, 3373.
Netzer, H. et al., 2007. ApJ 666, 806.

Netzer, H., 2009. MNRAS 399, 1907.

Netzer, H., 2015. ARA\&A 53, 365.

Nandra, K. et al., 2007. ApJL 660, 11.

Nandra, K. et al., 2013. < arXiv:1306.2307>.

Nandra, K. et al., 2015. ApJS 220, 10.

Oliver, S. J. et al., 2012. MNRAS 424, 1614.

Padovani, P. et al., 2017. A\&A Rev. 25, 2.

Page, M. J. et al., 2012. Nature 485, 213.

Page, M. J., Stevens, J. A., Ivison, R. J., Carrera, F. J., 2004. ApJL $611,85$.

Pannella, M. et al., 2009. ApJL 698, 116.

Paolillo, M. et al., 2017. MNRAS 471, 4398.

Paolillo, M., Schreier, E. J., Giacconi, R., Koekemoer, A. M., Grogin, N. A., 2004. ApJ 611, 93.

Papadakis, I. E., Chatzopoulos, E., Athanasiadis, D., Markowitz, A., Georgantopoulos, I., 2008. A\&A 487, 475.

Papovich, C. et al., 2006. ApJ 640, 92.

Papovich, C. et al., 2010. ApJ 716, 1503.

Pardo, K. et al., 2016. ApJ 831, 203.

Patel, S. G., Kelson, D. D., Williams, R. J., Mulchaey, J. S., Dressler, A., McCarthy, P. J., Shectman, S. A., 2015. ApJ 799, 17.

Peng, C. Y., 2007. ApJ 671, 1098.

Peng, C. Y. et al., 2006a. ApJ 649, 616.

Peng, C. Y., Impey, C. D., Ho, L. C., Barton, E. J., Rix, H.-W., 2006 b. ApJ 640, 114.

Peterson, B. M., 2001. in Advanced Lectures on the Starburst-AGN Connection, ed. I. Aretxaga, D. Kunth, R. Mújica (Singapore: World Scientific), 3.

Peterson, B., 2014. SSRv. 183, 253.

Pierce, C. M. et al., 2007. ApJL 660, 19.

Pierre, M. et al., 2017. AN 338, 334.

Plotkin, R. M. et al., 2016. ApJ 825, 139.

Prieto, M. A., Mezcua, M., Fernández-Ontiveros, J. A., Schartmann, M., 2014. MNRAS 442, 2145.

Ptak, A. et al., 2006. ApJ 637, 147.

Ptak, A. et al., 2007. ApJ 667, 826

Puccetti, S. et al., 2006. A\&A 457, 501.

Rafferty, D. A., Brandt, W. N., Alexander, D. M., Xue, Y. Q., Bauer, F. E., Lehmer, B. D., Luo, B., Papovich, C., 2011. ApJ 742, 3.

Raimundo, S. I., Fabian, A. C., Bauer, F. E., Alexander, D. M., Brandt, W. N., Luo, B., Vasudevan, R. V., Xue, Y. Q., 2010. MNRAS 408, 1714.

Ranalli, P., Comastri, A., Setti, G., 2003. A\&A 399, 39.

Ranalli, P. et al., 2013. A\&A 555, 42.

Reddy, N. A. et al., 2005. ApJ 633, 748.

Reddy, N. A., Steidel, C. C., Erb, D. K., Shapley, A. E., Pettini, M., 2006. ApJ 653, 1004.

Reines, A. E., Comastri, A., 2016. PASA 33, 54.

Reines, A. E. et al., 2014. ApJL 787, 30.

Reines, A. E., Greene, J. E., Geha, M., 2013. ApJ 775, 116

Reines, A. E., Sivakoff, G. R., Johnson, K. E., Brogan, C. L., 2011. Nature 470, 66.

Reines, A. E., Volonteri, M., 2015. ApJ 813, 82

Ricci, C., 2016. ApJ 820, 5.

Ricci, F., Marchesi, S., Shankar, F., La Franca, F., Civano, F., 2017. MNRAS 465, 1915.

Risaliti, G., Maiolino, R., Salvati, M., 1999. ApJ 522, 157.

Risaliti, G., Young, M., Elvis, M., 2009. ApJL 700, 6.

Robertson, B. E., Ellis, R. S., Dunlop, J. S., McLure, R. J., Stark, D. P., 2010. Nature 468, 49.

Robertson, B. E. et al., 2013. ApJ 768, 71.

Rodighiero, G. et al., 2015. ApJL 800, 10.

Rosario, D. J. et al., 2012. A\&A 545, 45.

Rosario, D. J. et al., 2013a. ApJ 771, 63. 
Rosario, D. J. et al., 2013b. ApJ 763, 59.

Rosati, P. et al., 2002. ApJ 566, 667.

Rovilos, E. et al., 2012. A\&A 546, 58.

Rowan-Robinson, M., 1995. MNRAS 272, 737.

Rumbaugh, N. et al., 2017. MNRAS 466, 496.

Runnoe, J. C., et al., 2016. MNRAS 455, 1691.

Sacchi, N. et al., 2009. ApJ 703, 1778.

Sajina, A., Yan, L., Fadda, D., Dasyra, K., Huynh, M., 2012. ApJ 757, 13.

Salvaterra, R., Haardt, F., Volonteri, M., 2007. MNRAS 374, 761.

Salvaterra, R., Haardt, F., Volonteri, M., Moretti, A., 2012. A\&A 545, L6.

Sanders, D. B., Soifer, B. T., Elias, J. H., Madore, B. F., Matthews, K., Neugebauer, G., Scoville, N. Z., 1988. ApJ 325, 74.

Sani, E., Marconi, A., Hunt, L. K., Risaliti, G., 2011. MNRAS 413 1479 .

Santini, P. et al., 2009. A\&A 504, 751.

Santini, P. et al., 2012. A\&A 540, 109.

Santini, P. et al., 2015. ApJ 801, 97.

Sartori, L. F. et al., 2015. MNRAS 454, 3722.

Satyapal, S., et al., 2014. ApJ 784, 113.

Schramm, M., Silverman, J. D., 2013. ApJ 767, 13.

Schramm, M. et al., 2013. ApJ 773, 150.

Schulze, A., Gebhardt, K., 2011. ApJ 729, 21.

Schulze, A., Wisotzki, L., 2011. A\&A 535, 87.

Schulze, A., Wisotzki, L., 2014. MNRAS 438, 3422.

Seth, A. C. et al., 2010. ApJ 714, 713.

Severgnini, P., Caccianiga, A., Della Ceca, R., 2012. A\&A 542, 46.

Shankar, F., 2009. NewAR 53, 57.

Shankar, F. et al., 2016. MNRAS 460, 3119

Shao, L. et al., 2010. A\&A 518, L26.

Shemmer, O. et al., 2006. ApJL 646, 29.

Shemmer, O. et al., 2008. ApJ 682, 81.

Shemmer, O. et al., 2014. ApJ 783, 116

Shen, Y., 2013. BASI 41, 61.

Shen, Y., Kelly, B. C., 2010. ApJ 713, 41.

Shi, Y., Helou, G., Armus, L., Stierwalt, S., Dale, D., 2013a. ApJ 764, 28.

Shi, Y., Helou, G., Armus, L., 2013b. ApJ 777, 6.

Shimizu, T. T., Mushotzky, R. F., Meléndex, M., Koss, M. J., Barger, A. J., Cowie, L. L., 2017. MNRAS 466, 3161.

Sijacki, D., Springel, V., Di Matteo, T., Hernquist, L., 2007. MNRAS $380,877$.

Silverman, J. D. et al., 2008a. ApJ 679, 118.

Silverman, J. D. et al., 2008b. ApJ 675, 1025

Silverman, J. D. et al., 2009. ApJ 696, 396.

Silverman, J. D. et al., 2010. ApJS 191, 124.

Silverman, J. D. et al., 2011. ApJ 743, 2.

Simcoe, R., McLeod, K. K., Schachter, J., Elvis, M., 1997. ApJ 489, 615.

Skelton, R. E. et al., 2014. ApJS 214, 24.

Smolčić, V. et al., 2017a. A\&A 602, 2.

Smolčić, V. et al., 2017b. A\&A 602, 6.

Sobolewska, M. A., Papadakis, I. E., 2009. MNRAS 399, 1597.

Spitler, L. R. et al., 2012. ApJL 748, 21.

Springel, V. et al., 2005. Nature 435, 629 .

Stark, A. A. et al., 1992. ApJS 79, 77.

Stern, D. et al., 2002. AJ 123, 2223.

Stern, D. et al., 2014. ApJ 794, 102

Straatman, C. M. S. et al., 2016. ApJ 830, 51.

Strateva, I. et al., 2001. AJ 122, 1861.

Suh, H., Hasinger, G., Steinhardt, C., Silverman, J. D., Schramm, M., 2015. ApJ 815, 129.

Sun, M. Y. et al., 2015. ApJ 802, 14.

Symeonidis, M. et al., 2011. MNRAS 417, 2239.
Symeonidis, M. et al., 2014. MNRAS 443, 3728.

Tanaka, M., Finoguenov, A., Ueda, Y., 2010. ApJL 716, 152.

Tanaka, M. et al., 2012. PASJ 64, 22.

Tanaka, M. et al., 2013. PASJ 65, 17.

Taylor, E. N. et al., 2009. ApJS 183, 295.

Tozzi, P., et al., 2006. A\&A 451, 457.

Treister, E., Urry, C. M., 2006. ApJL 652, 79.

Treister, E., Urry, C. M., 2012. AdAst 2012, 16

Treister, E., Urry, C. M., Virani, S., 2009a. ApJ 696, 110

Treister, E. et al., 2009b. ApJ 706, 535.

Treister, E. et al., 2009c. ApJ 693, 1713.

Treister, E., Schawinski, K., Urry, C. M., Simmons, B. D., 2012. ApJL $758,39$.

Tremaine, S. et al., 2002. ApJ 574, 740

Tremmel, M. et al., 2013. ApJ 766, 19.

Trevese, D., Boutsia, K., Vagnetti, F., Cappellaro, E., Puccetti, S., 2008. A\&A 488, 73.

Trouille, L., Barger, A. J., Cowie, L. L., Yang, Y., Mushotzky, R. F., 2008. ApJS 179, 1.

Trump, J. R. et al., 2011. ApJ 733, 60.

Tsujimoto, M. et al., 2011. A\&A 525, 25

Ueda, Y. et al., 2008. ApJS 179, 124.

Ueda, Y., Akiyama, M., Hasinger, G., Miyaji, T., Wastson, M. G., 2014. ApJ 786, 104.

Ulrich, M.-H., Maraschi, L., Urry, C. M., 1997. ARA\&A 35, 445.

Urrutia, T., Lacy, M., Spoon, H., Glikman, E., Petric, A., Schulz, B., 2012. ApJ 757, 125.

Uttley, P., McHardy, I. M., Papadakis, I. E., 2002. MNRAS 332, 231.

Vagnetti, F., Middei, R., Antonucci, M., Paolillo, M., Serafinelli, R., 2016. A\&A 593, 55.

Vagnetti, F., Turriziani, S., Trevese, D., 2011. A\&A 536, 84.

Valluri, M., Ferrarese, L., Merritt, D., Joseph, C. L., 2005. ApJ 628, 137.

van den Bosch, R. C. E., de Zeeuw, P. T., 2010. MNRAS 401, 1770.

van Dokkum, P. G. et al., 2006. ApJL 638, 59.

Vasudevan, R. V. et al., 2013. ApJ 763, 111

Vattakunnel, S. et al., 2012. MNRAS 420, 2190.

Vaughan, S., Edelson, R., Warwick, R. S., Uttley, P., 2003. MNRAS 345, 1271.

Vignali, C., Alexander, D. M., Comastri, A., 2006. MNRAS 373, 321.

Vignali, C., 2014. IAUS 304, 132.

Vignali, C. et al., 2014. A\&A 571, 34.

Vika, M., Driver, S. P., Cameron, E., Kelvin, L., Robotham, A., 2012. MNRAS 419, 2264.

Villforth, C. et al., 2014. MNRAS 439, 3342.

Villforth, C. et al., 2017. MNRAS 466, 812.

Villforth, C., Koekemoer, A. M., Grogin, N. A., 2010. ApJ 723, 737.

Vito, F. et al., 2013. MNRAS 428, 354.

Vito, F., Gilli, R., Vignali, C., Comastri, A., Brusa, M., Cappelluti, N., Iwasawa, K., 2014a. MNRAS 445, 3557.

Vito, F. et al., 2014b. MNRAS 441, 1059.

Vito, F. et al., 2016. MNRAS 463, 368.

Vito, F. et al., submitted. MNRAS.

Volonteri, M., 2010. A\&A Rev. 18, 279.

Volonteri, M., Reines, A. E., 2016. ApJL 820, 6.

Volonteri, M., Stark, D. P., 2011. MNRAS 417, 2085.

Wandel, A., 1999. ApJL 519, 39.

Wang, J. M., Watarai. K. Y., Mineshige. S., 2004. ApJL 607, 107.

Wang, W.-H., Cowie, L. L., \& Barger, A. J., 2004. ApJ 613, 655.

Wang, S. X. et al., 2013. ApJ 778, 179.

Wang, T. et al., 2017. A\&A 601, 63.

Wang, W.-H., Cowie, L. L., Barger, A. J., Keenan, R. C., Ting, H.-C., 2010. ApJS 187, 251.

Wardlow, J. L. et al., 2011. MNRAS 415, 1479.

Weiß, A. et al., 2009. ApJ 707, 1201. 
Weisskopf, M. C., Gaskin, J., Tananbaum, H., Vikhlinin, A., 2015. SPIE 9510, 2

Weisskopf, M. C., Tananbaum, H. D., van Speybroeck, L. P., O’Dell, S. L., 2000. Proc. SPIE 4012, 2.

Williams, R. E. et al., 1996. AJ 112, 1335.

Willott, C. J., 2011. ApJL 742, 8.

Wolf, C. et al., 2004. A\&A 421, 913.

Wolf, C., Hildebrandt, H., Taylor, E. N., Meisenheimer, K., 2008. A\&A 492, 933.

Worsley, M. A. et al., 2005. MNRAS 357, 1281.

Wu, J. et al., 2011. ApJ 736, 28.

Xue, Y. Q., Cui, W., 2005. ApJ 622, 160.

Xue, Y. Q. et al., 2010. ApJ 720, 368.

Xue, Y. Q. et al., 2011. ApJS 195, 10.

Xue, Y. Q. et al., 2012. ApJ 758, 129.

Xue, Y. Q., Luo, B., Brandt, W. N., Alexander, D. M., Bauer, F. E., Lehmer, B. D., Yang, G., 2016. ApJS 224, 15.

Xue, Y. Q., Yuan, F., Cui, W., 2006. ApJ 647, 194.

Yang, G. et al., 2014. ApJS 215, 27.

Yang, G. et al., 2016. ApJ 831, 145.

Yang, G. et al., 2017. ApJ 842, 72.

Yang, Q. X. et al., 2015. MNRAS 447, 1692.

Yencho, B., Barger, A. J., Trouille, L., Winter, L. M., 2009. ApJ 698, 380.

Younes, G. et al., 2011. A\&A 530, 149.

Young, M. et al., 2012. ApJ 748, 124.

Yuan, F., Narayan, R., 2014. ARA\&A 52, 529.

Zheng, X. C. et al., submitted. ApJ.

Zheng, X. Z. et al., 2009. ApJ 707, 1566.

Zhou, X.-L., Zhang, S.-N., Wang, D.-X., Zhu, L., 2010. ApJ 710, 16.

Zhou, X.-L., Zhao, Y. H., 2010. ApJL 720, 206.

Zhu, S. F., Xue, Y. Q., 2016. ApJ 825, 56.

Zibetti, S., Charlot, S., Rix, H.-W., 2009. MNRAS 400, 1181.

Zinn, P.-C., Blex, S., Seymour, N., Bomans, D. J., 2012. A\&A 547, 50 . 